\title{
DEFORMING HOMOTOPY EQUIVALENCES TO HOMEOMORPHISMS IN ASPHERICAL MANIFOLDS
}

\author{
BY P. E. CONNER ${ }^{1}$ AND FRANK RAYMOND ${ }^{1}$
}

\begin{abstract}
Aspherical manifolds are closed manifolds which are $K(\pi, 1)$ 's. They play a significant role in many branches of mathematics. This paper constructs "model" aspherical manifolds for various classes of $\pi$ and investigates and surveys their groups of homeomorphisms. It is not known whether aspherical manifolds having the same fundamental groups as our model manifolds can topologically differ from them. If $\pi$ contains a normal abelian subgroup then the model aspherical manifolds are special instances of injective Seifert fiber spaces. The group of (singular) fiber preserving homeomorphisms of Seifert fiber spaces are characterized and criteria obtained so that each self-homotopy equivalence may be deformed to such a homeomorphism. Many of our model aspherical manifolds satisfy these criteria. Other applications are given to group theory, differential geometry, complex manifolds as well as topology. We have also included a list of unsolved problems.
\end{abstract}

1. Introduction. This article, which is an expanded version of an hour address by the second author, is largely, but not exclusively, devoted to the study of aspherical manifolds. These are closed manifolds $M$ whose universal coverings are contractible. Thus, they are $K(\pi, 1)$ 's where $\pi$ is their fundamental group $\left(\pi=\pi_{1}(M)\right)$. Aspherical manifolds occur quite naturally in several complex variables (e.g., Riemann surfaces, quotients of bounded domains, hyperbolic manifolds, holomorphic Seifert fiberings), differential geometry (e.g., manifolds whose sectional curvature is $\leqslant 0$ ), Lie group theory (e.g., double coset spaces $K \backslash G / \Gamma$, where $G$ is connected, $K$ a maximal compact and $\Gamma$ a torsion free uniform subgroup), group theory (e.g., groups whose cohomological dimension is finite), number theory and algebraic geometry (e.g., "fiber varieties", Brieskorn varieties), and of course topology. (Specific contacts with these fields arise within this paper as either part of the theory or as illustrative examples.) This has led to their study by many diverse methods. Consequently, the exploration of topological properties and their topological identification and classification takes on added significance. We may ask which groups $\pi$

An invited address presented to the Society on November 3, 1973 at Minneapolis, Minnesota under the title, Deforming self-homotopy equivalences to homeomorphisms in certain aspherical manifolds; received by the editors July 7, 1975.

AMS (MOS) subject classifications (1970). Primary 57-02, 57A10, 57A15, 57D50, 57E05, 57E10, 57E15, 57E30, 55F55, 55F65, 55C35, 22E40, 18H10, 20E40, 20J05, $20 \mathrm{H} 15$.

Key words and phrases. Aspherical manifolds, group extensions, automorphism group, Seifert fiber spaces, covering spaces, fiber preserving homeomorphisms, geometric realization, flat manifolds, Bieberbach classes, properly discontinuous actions, uniform discrete subgroups of Lie groups, self-homotopy equivalences, group of homeomorphisms.

${ }^{1}$ Supported in part by the National Science Foundation. 
appear as the fundamental groups of aspherical manifolds and how we may give a complete list of such manifolds. Essential progress can and has been made on various aspects of these problems.

To effectively study aspherical manifolds one needs to understand the relationship of the group of homeomorphisms of $M, \mathcal{H}(M)$, to the $H$-space of self-homotopy equivalences of $M, \mathcal{E}(M)$. This is investigated in $\S \S 2$ and 3 . It is recalled how $\mathscr{E}(M)$, which is explicitly determined by $\pi$ alone, also determines the compact Lie subgroups of $\mathcal{H}(M)$. In particular, we point out how this led to the construction of manifolds without any periodic maps.

To begin an attack on the classification problem we shall assume that $\pi$ has a normal subgroup $\Gamma$ which itself is the fundamental group of an aspherical manifold. Now, if the quotient group, $\pi / \Gamma=N$, is "something like" a (not necessarily torsion free) uniform discrete subgroup of a Lie group, we may construct, for each possible $\pi$, an actual specific aspherical manifold $M^{m}(\pi)$. Each $M^{m}(\pi)$ can be regarded as a "model" aspherical manifold for the group $\pi$. They usually enjoy rather spectacular topological properties. (For example, if $\Gamma$ is abelian and $N$ is finite our construction yields precisely all the flat manifolds. Many of the standard properties of flat manifolds may be deduced directly from the topological construction.) Moreover, no examples of aspherical manifolds are known that have such "allowable" normal subgroups $\Gamma$ and yet topologically differ from any of the model manifolds constructed herein.

It is possible to organize the study into 2 parts. The first, taken up in $\S 4$, has the center of $\Gamma$ trivial. This case will be developed further in a subsequent publication. The second is reduced to $\Gamma$ itself being abelian. The remaining sections are devoted to this case.

If $\Gamma$ is abelian the construction of model aspherical manifolds is based upon the idea of Seifert fiberings, $\S 5$. These are mappings $\mu: X \rightarrow Y$ very much like fiber bundle mappings. However, the "fibers" $F_{y}=\mu^{-1}(y)$ may vary from point to point, but not arbitrarily. Each "fiber" $F_{y}$ is homeomorphic to some flat manifold. The space $Y$ and the group $N$ parameterizes the fibers and their fundamental groups. This construction does not depend upon asphericity and so much of what we do in $\$ \S 5$ through 9 is valid for arbitrary spaces.

To make this more concrete suppose that $(W, N)$ denotes a properly discontinuous action (not necessarily free) of a discrete group $N$ on a simply connected space $W$. For each arbitrary group extension of the form

$$
1 \rightarrow \mathbf{Z}^{k} \rightarrow \pi \rightarrow N \rightarrow 1
$$

one may construct an action of the semidirect product $T^{k} \circ N$ on $T^{k} \times W$ in which the group $N$ appears as a group of branched covering transformations and $T^{k}$ is the $k$-dimensional torus. There is induced a mapping, $\mu: X$ $=T^{k} \times W / N \rightarrow W / N=Y$, with $F_{y}=\mu^{-1}(y)=\mu^{-1}\left(w^{*}\right)=\left(T^{k} \times w\right) / N_{w}$, where $w^{*}$ denotes the orbit of $N$ through $w \in W$, and $N_{w}$ is the stabilizer of the action of $N$ at $w \in W$. The correspondence between (congruent) extensions and (equivalent) actions of $T^{k} \circ N$ on $T^{k} \times W$ is a natural bijective correspondence. The theory becomes practical when the induced covering action of $N$ on $T^{k} \times W$ is unbranched. This turns out to be the same as having each induced action $N_{w} \times\left(w \times T^{k}\right) \rightarrow\left(w \times T^{k}\right)$ free. The resulting mapping $\mu$ is then equivalent to what is called an injective Seifert fibering, and $\mu^{-1}\left(w^{*}\right)$ $=\left(w \times T^{k}\right) / N_{w}$ is a flat manifold. 
As a first illustration consider a compact Kaehler manifold $M$. If $M$ admits a connected compact group of holomorphic automorphisms then the orbit map $M \rightarrow M^{*}$ is a (holomorphic) injective Seifert fibering. This structure also enables one to fiber $M$ over a torus (and even holomorphically fiber it over a complex torus after a possible deformation). Moreover, given one such manifold the theory usually (but not always) produces an infinite number of other similar complex examples all topologically distinct from the original. See 5.7(c).

For a second illustration take an action $(W, N)$ where $W$ is an arbitrary contractible manifold with $W / N$ compact. Then, corresponding to each extension (*), with $\pi$ torsion free, the construction yields a model aspherical manifold $M(\pi)$ which is an (injective) Seifert fiber space having $\pi_{1}(M(\pi))=\pi$.

We have mentioned that these models often possess "spectacular" properties. In particular, we wish to determine if it is possible to represent the outer automorphisms of $\pi$ in (*) by self-homeomorphisms of these models.

An explicit formula for Aut $\pi$ and Out $\pi$ is determined in terms of $\mathbf{Z}^{k}$ and $N$ in $\S 6$. This is purely group theoretical and may be read completely out of context.

In $\S 7$, we show that the group of automorphisms of Seifert fiberings ("fiber" preserving homeomorphisms) is the geometric analogue of $\$ 6$. Here certain subgroups of Out $\pi$ can always be realized by automorphisms of Seifert fiber spaces. In $\$ 8$ a necessary and sufficient condition that the full group Out $\pi$ can be so geometrically realized is given. We then describe a large class of our model aspherical manifolds for which this condition can be verified. (No counterexamples are known.) This means our model manifolds possess extremely nice properties such as: Every self-homotopy equivalence can be deformed to a (fiber preserving) homeomorphism.

To motivate the text we have included many examples and introduced, in what we hope is a natural manner, some central problems concerning aspherical manifolds. These problems are, perhaps, ideal for (topological) surgery theory, although we have used none herein. It is reasonable to expect, in the near future, that surgery may be able to detect negative solutions to some of the problems posed in the text. We have also tried to make the material reasonably self-contained whenever feasible. References to details, when they exist in a satisfactory form elsewhere, are given. In many ways this paper is a sequel to [13] but no knowldege of [13] is required. The reader does need to know the theory of covering spaces, extensions of groups and some, but not much, algebraic topology.

\section{Section Headings}

§2. Definitions, Examples, and Problems 1 and 2.

$\S 3$. How $\pi$ Helps Determine the Compact Subgroups of the Automorphisms of $M^{m}$.

§4. The Construction of Aspherical Manifolds: $\pi$ with Centerless Normal Subgroups.

§5. Seifert Fiber Spaces. The Construction of Aspherical Manifolds: $\pi$ with Normal Abelian Subgroups.

§6. Computation of Out $\pi$. 
§7. Fiber Preserving Homeomorphisms and Geometrically Realizing the Subgroup $K$ of Out $\pi$.

§8. Deforming Homotopy Equivalences to Fiber Reserving Homeomorphisms.

§. The Domination of $\mathscr{E}(M)$ by $\mathcal{H}(M)$ and the Geometric Realization of Finite Subgroups of Out $\pi$.

We would like to express our appreciation to David Wigner for several suggestions and arguments and many fruitful conversations.

2. Definitions, examples, and problems 1 and 2 . A closed manifold $M$ is called aspherical if $M$ is connected and its universal covering $\tilde{M}$ is contractible. Since $\pi_{i}(\tilde{M})=0, \pi_{i}(M)=0$, for all $i>1$. Hence, $M$ is a $K(\pi, 1)$-space, where $\pi=\pi_{1}(M)$. Now among spaces having the homotopy type of a C-W complex the $K(\pi, 1)$ 's are the spaces whose homotopy type is completely determined by the fundamental group alone. Thus one would suspect that if the $K(\pi, 1)$-space is also a closed manifold, then $\pi$ might come close to determining the topology of the manifold. This leads one to perhaps the most difficult and important problem concerning aspherical manifolds.

2.1. Problem 1-A. Let $M$ and $N$ be aspherical manifolds with $\pi_{1}(M)$ isomorphic to $\pi_{1}(N)$. Are $M$ and $N$ homeomorphic?

Since any isomorphism of the fundamental groups $\gamma: \pi_{1}(M) \cong \pi_{1}(N)$ may be geometrically realized by the induced isomorphism on the fundamental group of a homotopy equivalence $g: M \rightarrow N$, the problem may be stated perhaps more interestingly as follows:

2.2. Problem 1-B. Let $g: M \rightarrow N$ be a homotopy equivalence between aspherical manifolds. Is $g$ homotopic to a homeomorphism?

One could go out on a limb and conjecture that the answer to Problem 1-B is always affirmative since no examples counter to this conjecture are known. However it must be warned that the truth of this conjecture in dimensions 3 and 4 would imply the truth of the Poincare conjecture in these dimensions. This problem is linked to surgery and to a modified form of a conjecture of $\mathrm{S}$. P. Novikov on "Improved higher signatures" in Manifolds-Tokyo 1973, Univ. of Tokyo Press, edited by A. Hattori. See the section, "Some problems in topology", pp. 423-424.

Since the problem seems so intractible one needs to impose more hypotheses to make any progress. One of the most striking results is that of C. T. C. Wall: A group $\pi$ is Z-polycyclic if $\pi$ is a finitely iterated extension of the infinite cyclic group Z. Wall has shown [44] that if $\pi$ is Z-polycyclic, there exists an aspherical manifold with fundamental group $\pi$. Furthermore, in this class of Z-polycyclic groups Problem 1-B has an affirmative solution in all dimensions other than 3 or 4 . Fortunately, this class of manifolds is very interesting in its own right, but unfortunately the class is rather "thin" among all the possible aspherical manifolds. See also [26].

Another approach to Problem 1-B is to make $M=N$ and consider selfhomotopy equivalences.

Problem 1-C. Let $g: M \rightarrow N$ be a self-homotopy equivalence. Is $g$ homotopic to a homeomorphism?

We shall investigate this in $\S \S 7,8$ and 9 for $\pi$ which have finitely generated abelian normal subgroups. 
2.3. To dispel any notion that aspherical manifolds are an exotic class of manifolds we offer the following short list of examples.

1. LOW DIMENSIONAL EXAMPLES.

(a) dimension $1, S^{1}$;

(b) dimension 2, all closed 2-manifolds except the 2-sphere and the real projective plane;

(c) dimension 3, all closed oriented 3-manifolds $M$ such that $\pi$ is not isomorphic to a nontrivial free product, a finite group, nor $\mathbf{Z}$.

2. All closed Riemannian manifolds whose sectional curvature is $\leqslant 0$.

3. The double coset spaces $K \backslash G / N$ where $N$ is a discrete torsion free uniform subgroup of a connected Lie group $G$ and $K$ is a maximal compact subgroup. (The coset space $K \backslash G$ is diffeomorphic to a Euclidean space and any discrete uniform subgroup will act on the right so that the orbit map $(K \backslash G, N) \stackrel{I N}{\longrightarrow}(K \backslash G) / N$ is a regular branched covering map. This is unbranched if and only if $N \cap K=e \in G$ which is the same as saying that $N$ is torsion free.)

4. A set of generators of the unoriented bordism ring $\Re^{*} \cdot \Re^{*}$ is generated as a graded polynomial ring over $\mathbf{Z} / 2 \mathbf{Z}$ by aspherical manifolds. Moreover, these generators for all dimensions greater than 2 all contain a nontrivial center in their fundamental group and admit an action of the circle without fixed points. See [10].

5. As mentioned above, if $\pi$ is poly $\mathbf{Z}$ (a finite iterated extension of $\mathbf{Z}$ ) there exists an aspherical manifold $M^{m}$ with fundamental group $\pi$. This manifold is an iterated fibering over the circle and except possibly in dimensions 3 and 4 the homeomorphism type of $M^{m}$ is completely determined by the group $\pi$ [44].

2.4. All of the examples (except perhaps in 1(c) are covered by Euclidean space. This leads one to conjecture that the solution to the next problem may be affirmative.

Problem 2. If $M$ is aspherical is the universal covering $\tilde{M}$ homeomorphic to Euclidean space?

This is a much more tractable problem (except in dimensions 3 and 4 where, once again, the truth of the conjecture would imply the truth of the Poincare conjectures in each of these dimensions) due to the fact that it is known to be only a problem about the group $\pi$. The most general results known appear to be by J. Stallings [41], R. Lee and F. Raymond [30], and F. E. A. Johnson [25].

THEOREM 1 [30]. If $M^{m}$ is aspherical and $\pi$ contains a finitely generated nontrivial normal abelian subgroup, then the universal covering of $M$ is homeomorphic to $\mathbf{R}^{m}, m \neq 3$ or 4 .

The general principle which makes the argument employed in the proof of Theorem 1 work is described in $[30,6.3]$. By the end of this paper we will have described many situations under which the general principle can be applied. Consequently, Theorem 1 will be considerably extended.

Problem 2 and Theorem 1 offer another justification for studying aspherical manifolds. If the universal covering of $M^{m}$ is Euclidean space then $\tilde{M}^{m}$ must have a very simple cell structure. The complicated cell structure of $M$ all "untwists" in the universal covering and all the homotopy theoretic properties of $M$ are functions of $\pi$ alone. The "combinatorial" structure of $M^{m}$ then is 
obtained from the way the group $\pi$ acts as covering transformations on Euclidean space. In some real sense this means that aspherical manifolds should be the simplest of all types of manifolds. One might counter with spheres as being simpler since one assembles them with only 2 cells sewn together in an elementary way. But, one may ask, what are the homotopy groups of spheres?

3. How $\pi$ helps determine the compact subgroups of the automorphisms of $M^{m}$. We now wish to illustrate further how the group $\pi$ determines certain aspects of the topology of $M$. A good starting place for investigating any mathematical object is the set of automorphisms of the object.

3.1. Let $\mathscr{E}(X)$ denote the space of self-homotopy equivalences of a space $X$, and $\mathcal{H}(X)$ the group of homeomorphisms of $X$. There is a natural inclusion $i: \mathfrak{H}(X) \rightarrow \mathcal{E}(X)$ which is an $H$-space homomorphism. (We assume that our spaces are always "reasonable", which will actually be the case for all our applications, so that no pathology arises from the compact open topology. Certainly pathology will be avoided if we assume that our spaces are locally compact, separable metric ANR's; see [21, Theorem 4.1].) There are two cases to consider.

(a) THe BASED CATEgory. All maps and homotopies fix a base point $x_{0} \in X$.

(b) The UNBASED CATEGORY. The maps and/or homotopies are not required to $f i x$ a base point $x_{0} \in X$.

Let $\mathcal{E}\left(X ; x_{0}\right)$ be the subspace of $\mathscr{E}(X)$ for which each homotopy equivalence fixes $x_{0}$. Let the automorphisms of $\pi_{1}\left(X, x_{0}\right)$ be denoted by Aut $\pi_{1}\left(X, x_{0}\right)$ and the inner automorphisms by Inn $\pi_{1}\left(X, x_{0}\right)$. Let Out $\pi_{1}\left(X, x_{0}\right)$, the outer automorphism group, be the quotient group, Aut $\pi_{1}\left(X, x_{0}\right) / \operatorname{Inn} \pi_{1}\left(X, x_{0}\right)$.

For any self-homotopy equivalence $f:\left(X, x_{0}\right) \rightarrow\left(X, x_{0}\right)$, we induce an automorphism $f_{*}: \pi_{1}\left(X, x_{0}\right) \rightarrow \pi_{1}\left(X, x_{0}\right)$. If $f$ is based homotopic to $g$ the automorphisms agree and yield a representation

$$
\theta: \pi_{0}\left(\mathcal{E}\left(X ; x_{0}\right), 1_{X}\right) \rightarrow \text { Aut } \pi_{1}\left(X, x_{0}\right) .
$$

In the unbased case, we choose, arbitrarily, paths $\beta_{x}$ from $x_{0}$ to an arbitrary $x$. For $f:\left(X, x_{0}\right) \rightarrow(X, x)$ and a loop $\alpha:(I, 0,1) \rightarrow\left(X, x_{0}, x_{0}\right)$, we get the loop $\beta_{x}^{-1} f(\alpha) \beta_{x}$ based at $x_{0}$. (We read composition of paths from right to left as with functions.) Thus, we have a map $\Psi: \mathcal{E}(X) \rightarrow$ Aut $\pi_{1}\left(X, x_{0}\right)$ which assigns to each self-homotopy equivalence an automorphism of $\pi_{1}\left(X, x_{0}\right)$. If $g$ is homotopic to $f$ the automorphisms will agree modulo an inner automorphism. Hence we receive a $H$-space homomorphism, $\Psi: \mathcal{E}(X) \rightarrow$ Out $\pi_{1}\left(X, x_{0}\right)$, which factors through the representation,

$$
\Psi_{*}: \pi_{0}\left(\mathcal{E}(X), 1_{X}\right) \rightarrow \text { Out } \pi_{1}\left(X, x_{0}\right) .
$$

These representations are just one aspect of the following. The evaluation map $\omega: \mathcal{E}(X) \rightarrow X$ defined by $\omega(h)=h\left(x_{0}\right)$ is a fibration in the sense of Hurewicz $\left[23\right.$, p. 83]. The fiber is nothing but $\&\left(X ; x_{0}\right)$. The homotopy exact sequence for the fibering (with connected $X$ ) for low degrees is 


$$
\begin{aligned}
\cdots \rightarrow \pi_{1}\left(\mathscr{E}(X), 1_{X}\right) & \rightarrow \pi_{1}\left(X, x_{0}\right) \\
& \rightarrow \pi_{0}\left(\mathscr{E}\left(X ; x_{0}\right), 1_{X}\right) \rightarrow \pi_{0}\left(\mathscr{E}(X), 1_{X}\right) \rightarrow 1 .
\end{aligned}
$$

For $X$ which are $K(\pi, 1)$ 's the representations $\theta$ and $\Psi_{*}$ are isomorphisms. Moreover, if $X$ has the homotopy type of a finite complex then the groups

$$
\pi_{i}\left(\mathscr{E}(X), 1_{X}\right)=\pi_{i-1}\left(\mathscr{E}\left(X ; x_{0}\right), 1_{X}\right)=0, \quad i>1,
$$

and $\pi_{1}\left(\mathscr{E}(X), 1_{X}\right)=\mathscr{Z}(\pi)$, the center of $\pi$ [20]. Hence, with a little care, the homotopy exact sequence becomes the well-known exact sequence of group theory:

$$
1 \rightarrow \mathscr{Z}(\pi) \rightarrow(\pi)=\pi_{1}\left(X, x_{0}\right) \rightarrow \text { Aut } \pi_{1}\left(X, x_{0}\right) \rightarrow \text { Out } \pi_{1}\left(X, x_{0}\right) \rightarrow 1 .
$$

This means that $\mathscr{E}(X)$ has the homotopy type of $K(\mathscr{Z}(\pi), 1) \times$ Out $\pi$. This immediately raises our next

Problem 3. If $X$ has the homotopy type of a finite complex and $X$ is a $K(\pi, 1)$, is $\mathscr{Z}(\pi)$ finitely generated?

If $\mathscr{Z}(\pi)$ is finitely generated and has rank $k \geqslant 0$, then $\mathscr{E}(X)$ has the homotopy type of $T^{k} \times$ Out $\pi$, where $T^{k}$ is the $k$-dimensional torus. It is unlikely that the answer to Problem 3 will be affirmative. Therefore, we probably instead should state the problem in terms of an aspherical manifold.

3.2. Let $\mathcal{H}(X)$ be the subspace of $\mathscr{E}(X)$ consisting of the homeomorphisms of $X$. It is a topological group under our reasonable assumptions on the space $X$. The inclusion $i: \mathcal{F C}(X) \rightarrow \mathcal{E}(X)$ is an $H$-space homomorphism. For $X=M$, an aspherical manifold, this leads to the difficult and significant

PROBLEM 4. Is $i$ a homotopy equivalence?

The answer is known only when $m \leqslant 2$ (affirmative). In dimension 3 it is known from Waldhausen's work that $i$ induces an isomorphism on the 0 homotopy groups, that is, on the group of path components provided that the manifolds are "sufficiently large" and irreducible [43]. No other general information seems to be known. Much of what we have to say in this article deals directly with finding conditions on certain $M$ that imply $i_{*}$ is an epimorphism on the homotopy group level.

ADDED IN PROOF. It has recently been shown by A. Hatcher and W. C. Hsiang and $\mathrm{R}$. Sharpe that $i$ is not a monomorphism on $\pi_{0}$ for a class of aspherical manifolds which include tori.

Allen Hatcher in Homeomorphisms of sufficiently large $\mathbf{P}^{2}(\mathbf{R})$ irreducible 3manifolds (to appear in Topology) has shown that $\pi_{1}\left(\mathfrak{F C}\left(M^{3}\right)\right) \cong$ center of $\left(\pi_{1}\left(M^{3}\right)\right)$ and $\pi_{i}\left(\left(M^{3}\right)\right)=0, i>1$, for the manifolds mentioned in the title. His results are also valid in the PL category. Our results (7.6 (Theorem 9), 7.8, 8.3(b)-4, and $\$ 9(2.4))$ then imply that the group of fiber preserving diffeomorphisms is a deformation retract of $\mathcal{H C}\left(M^{3}\right)$ for 3-dimensional Seifert fiberings. Corollary 2 of 6.3 already gave a very explicit formula for $\pi_{0}\left(\mathcal{H}\left(M^{3}\right)\right)$ by virtue of $[43]$.

3.3. Let $G$ be a compact Lie group embedded as a closed subgroup of the group of homeomorphisms, $\mathcal{H C}(M)$, of the aspherical manifold $M^{m}$. That is, we assume that $G$ acts effectively on $M^{m}$. Let $G_{0}$ denote the connected component 
containing the identity of $G$. The following result imposes strong restrictions on the nature of $G$ and is very relevant to Problem 4.

THEOREM 2. (a) The based case. $G_{0}$ must be the identity and the representation $\theta \circ i: G \rightarrow$ Aut $\pi_{1}\left(M, x_{0}\right)$ is a monomorphism.

(b) The unbased case. (i) $G_{0} \cong T^{k}$, for some $k$, where $0 \leqslant k \leqslant \mathscr{Z}(\pi)$.

(ii) All stability groups of $G_{0}$ are finite subgroups of $T^{k}$, consequently, the Euler characteristic $\chi(M)$ must be 0 , when $k>0$.

(iii) If $\mathscr{Z}(\pi)=0$, then the representation $\Psi \circ i: G \rightarrow \pi_{1}\left(M, x_{0}\right)$ is a monomorphism.

The faithfulness of the representation $\Psi \circ i$ is $A$. Borel's Theorem and is proved in $[17, \S 3]$. The rest of the theorem can be found in [11, $\S 5,6$, and 7]. The key to the arguments concerning $G_{0}$ is the monotonicity of the evaluation homomorphism on the fundamental groups. Let $(G, X)$ be an action of a path connected group on $X$. For each $x \in X$ define the evaluation map

$$
\mathrm{ev}^{x}:(G, e) \rightarrow(X, x)
$$

by $\mathrm{ev}^{x}(g)=g(x)$. This induces the evaluation homomorphism

$$
\mathrm{ev}_{*}^{x}: \pi_{1}(G, e) \rightarrow \pi_{1}(X, x) .
$$

The image is a central subgroup of $\pi_{1}(X, x)$ and is "independent" of the base point (as long as $X$ is path connected). For aspherical manifolds, one proves that $\mathrm{ev}_{*}^{x}$ is a monomorphism. From this fact one deduces the results. See [11, $\S \S 5,6$ and 7] for complete details.

The theorem shows that $\pi$ gives bounds on the size of any compact $G$. In particular, $G$ is finite whenever it acts with fixed points, $\chi(M) \neq 0$, or the center of $\pi$ is trivial. Well-known examples of aspherical manifolds for which the center is known to be trivial are:

1. Closed Riemannian manifolds with sectional curvature less than 0 [4].

2. $M$, for which $\chi(M) \neq 0$ [20], [42].

3. Any closed 2-manifold with genus $<0$; any irreducible aspherical 3manifold which admits no action of the circle.

4. $K \backslash G / N$, where $G$ is the adjoint form of a semisimple Lie group without compact factors and $N$ is a uniform discrete torsion free subgroup of $G$.

3.4. Manifolds with no actions. Certainly, if one can compute Aut $\pi$ or Out $\pi$ one can then hope to build manifolds with very few or no actions of finite groups. It had been long conjectured that "most" manifolds should exhibit little or no symmetry. That is, the only finite subgroups of $\mathcal{F C}(M)$ would be the trivial group. In [17] and [18] methods for computing Aut $\pi$ and Out $\pi$ are developed and the following examples are constructed.

THEOREM 3. There exist aspherical manifolds $M^{m}$ in all dimensions greater than 2 for which the only actions of finite groups are involutions. The fixed point set is always a disjoint collection of circles. Furthermore if $m>3$, all $M^{m}$ can be chosen to be orientable with all involutions preserving orientation for $m$ odd and reversing it for $m$ even. There exist aspherical 4-manifolds on which every finite group of $\mathcal{H}(M)$ acts freely. There exist in dimensions 7, 11, 16, 22, 29, and 37 aspherical manifolds which admit no effective actions of any finite groups. 
There exist aspherical 4-manifolds on which every finite group of $\mathcal{T C}(M)$ acts freely.

There exist in dimensions $7,11,16,22,29$, and 37 aspherical manifolds which admit no effective actions of any finite groups.

All the examples constructed above have solvable fundamental groups. In fact, in the last set of examples we construct an infinite number of distinct 7 dimensional solvmanifolds for which Out $\pi$ contains no elements of finite order. With care one can actually make Out $\pi=1$ for some of the 7dimensional examples. In 6.12 we shall give the computation of Out $\pi$ to yield the first assertion of Theorem 3.

3.5. No examples of simply connected closed manifolds without periodic maps are yet known. Of course one can find oriented compact simply connected 8-manifolds $W$ with our 7-dimensional examples as boundary. These manifolds cannot have any periodic maps. It is tempting to match two copies of $W$ along their boundaries and then form the connected sum with complex projective 4-space, $\mathrm{CP}_{4}$.

Problem 5. Does the resulting closed simply connected smooth 8-manifold admit any action of a finite group?

3.6. E. Bloomberg has studied the connected sum of aspherical manifolds. The resulting manifolds are no longer aspherical, but nevertheless by choosing distinct pairs of the 4-dimensional examples of Theorem 3, he has also been able to construct closed 4-manifolds (not aspherical) without any actions of finite groups [5].

Recently, F. Raymond and J. Tollefson have found orientable aspherical 3manifolds $M^{3}$ which admit no actions [38]. One may then apply a very strong result of P. K. Kim and J. Tollefson [35, Corollary 1] to conclude that $X \# M^{3}$ admits no $P L$ involution for any closed prime 3-manifold $X$ different from $S^{2} \times S^{1}$ or $M^{3}$.

[5] and [35] indicate that analysis of aspherical manifolds leads to interesting results on more complicated classes of manifolds. By using a cohomological version of some of the techniques employed in [5], one may show the following:

THEOREM 4. The connected sum $M_{1} \# \cdots \# M_{s}, s>1$, of any aspherical $n$ manifolds $n>1$, does not admit any effective action of the circle.

If this is now coupled with Conner's result that the unoriented bordism ring $\Re_{*}(p t)$ is generated by aspherical manifolds we have the

3.7. CoROLlary. If $M$ is a closed n-manifold, then there exists closed connected $n$-manifolds $N$, bordant to $M$, with the property that every compact Lie subgroup of $\mathcal{H C}(N)$ is finite.

This corollary can be interpreted as a form of the oft repeated quip, "Most manifolds should exhibit little or no symmetry".

4. The construction of aspherical manifolds: $\pi$ with centerless normal subgroups.

4.1. There are two difficult and distinct problems envisioned in the title of this section.

PROBLEM 6. Which groups $\pi$ can be the fundamental group of an aspherical manifold? 
Problem 7. If $\pi$ is the fundamental group of an aspherical manifold can we give an actual explicit construction of an aspherical manifold for the group $\pi$ ?

There are some very general and abstract criteria contributing to our knowledge of the first problem. For example, $\pi$ must be a Poincare duality group in the sense of Johnson and Wall [27] and Bieri [1], [2], [3]. In particular, $\pi$ must be torsion free, finitely presented, have cohomological dimension finite, satisfy Poincare duality in the strongest possible sense (that is $K(\pi, 1)$ is a finite Poincaré complex). These conditions impose strong restrictions on $\pi$ (see the papers mentioned above) but a complete and specific list of such groups is not possible at this time.

One is led to treat certain portions of the problems which arise naturally in some special algebraic or geometric way. With this in mind Problem 7 takes on added value. A feature therefore of the examples that we construct should exhibit interesting geometric and algebraic properties. Furthermore each constructed aspherical manifold $M^{m}(\pi)$ can be regarded as a model for all the other aspherical manifolds with the same fundamental group (if there are any others!) and give a hold to focus on Problem 1-B. The most comprehensive answer for special $\pi$ are those $\pi$ which are iterated extensions of $\mathbf{Z}$ (poly $\mathbf{Z}$ groups); see C. T. C. Wall [44] and the examples of 2.3. Part of our purpose here is to partially answer Problems 6 and 7.

4.2. Certainly a reasonable attempt should be made on those groups $\pi$ which contain a normal subgroup $\Gamma$ which is the fundamental group of an aspherical manifold $X$. Consider groups $\pi$ of the form

$$
1 \rightarrow \Gamma \rightarrow \pi \rightarrow N \rightarrow 1 \text {. }
$$

If $N$ is the fundamental group of an aspherical manifold $Y$ then one would suspect that one could construct an aspherical manifold $M(\pi)$ which would fiber over $Y$ with fiber $X$ and whose homotopy exact sequence at the fundamental group level is $(*)$. Such a construction is not known in general but it can be affected in certain special cases as we shall point out later. But first let us restrict the group $\Gamma$ but widen the possibilities alloted to $N$. Let us suppose for the rest of $\S 4$ that $N$ operates properly discontinuously on a contractible manifold $W$ with $W / N$ compact. Thus the orbit map $\nu: W \rightarrow W / N$ is a branched covering map and branching occurs when $N$ has elements of finite order only. Of course an important example to keep in mind is a not necessarily torsion free uniform discrete subgroup $N$ of a Lie group $G$. On $K \backslash G$, where $K$ is a maximal compact subgroup, $N$ acts properly discontinuously and $W / N$ corresponds to $K \backslash G / N$.

(This type of example is not as innocuous as it may appear at first glance. We would like to pose at least the following.

Problem 8. If $M$ is aspherical, does there exist a Lie group $G$ (with at most a finite number of connected components) and a torsion free uniform discrete subgroup $\pi$ so that $M$ is homeomorphic to the double coset space $K \backslash G / \pi$ ?)

4.3. Now let $X$ be an aspherical manifold with fundamental group $\Gamma$ and let us assume that

(i) $\Gamma$ has trivial center,

(ii) for $\phi: N \rightarrow$ Out $\Gamma$, which completely determines (*) up to congruence, there exists a homomorphism $\tilde{\phi}: N \rightarrow \mathcal{H}(X)$ so that $\Psi \circ i \circ \tilde{\phi}=\phi$. Then, 
THEOREM 5. The group $\pi$ is the fundamental group of an aspherical manifold $M(\pi)$ if and only if the extension $\pi$ is torsion free.

Thus, under the hypothesis, the crudest necessary condition on $\pi$ (that $\pi$ be torsion free) is also sufficient for $\pi$ to be the fundamental group of an aspherical manifold.

To prove our theorem we shall rely upon [11, §2]. Let us define a properly discontinuous action of $N$ on $P=X \times W$ by

$$
(x, w) \times \alpha \rightarrow(x, w) \cdot \alpha=\left(\tilde{\phi}\left(\alpha^{-1}\right) x, w \cdot \alpha\right) .
$$

Choose base points $x_{0} \in X, w_{0} \in W$ and $x_{0} \times w_{0}=p_{0} \in P$. For each $x$ $\in X, w \in W$, choose paths $\beta(t)$ and $\delta(t)$ beginning at $x_{0}$ or $w_{0}$ and terminating at $x$ or $w$, respectively. The path $(\beta(t), \delta(t))$ begins at $\left(x_{0} \times w_{0}\right)$ and terminates at $p=(x \times w)$. Each homeomorphism $(x, w) \rightarrow(x, w) \cdot \alpha$ defines an automorphism of $\pi_{1}\left(P, p_{0}\right)$ using the paths $(\beta(t), \delta(t))$. The automorphism depends upon the choice of paths but only up to inner automorphism. Thus one gets a homomorphism $N \rightarrow$ Out $\pi_{1}\left(P, p_{0}\right)=$ Out $\Gamma$. By the construction of $\S 2$ of [17] we see that this homomorphism agrees with $\phi$. Furthermore, on the universal covering $\tilde{P}=\tilde{X} \times W$ we get an action of $\pi$ where $\pi$ satisfies (*) up to a congruence. We remark that we must make one change from $[17, \S 2]$ where the actions are all given on the left. Since we want right actions we turn to $[14, \S 3.10]$ where the formulae in terms of left actions are turned into formulas for actions on the right. Notice that since the center of $\Gamma$ is trivial the extension, up to congruence, is determined only by $\phi$. We still wish to determine when the action of $\pi$ on $\tilde{P}$ are covering transformations or what is the same, when the action of $N$ on $P$ are covering transformations. Let $\alpha \in N_{w}$, the stabilizer of $w$, for some $w \in W$. Then $(x, w) \cdot \alpha=\left(\tilde{\phi}(\alpha)^{-1} x, w\right)$ and this equals $(x, w)$ if and only if $\phi\left(\alpha^{-1}\right) x=x$. But the right action defined by $N_{w} \times X \rightarrow X, x \rightarrow \tilde{\phi}\left(\alpha^{-1}\right) x$, must be free if $(x, w) \cdot \alpha \neq(x, w)$, for all $\alpha \in N_{w}$. Just as before the action, free or not, determines an extension $1 \rightarrow \Gamma \rightarrow \pi_{w} \rightarrow N_{w} \rightarrow 1$. This extension is clearly that induced by (*) and the inclusion $N_{w} \hookrightarrow N$. This means we have the commutative diagram of extensions:

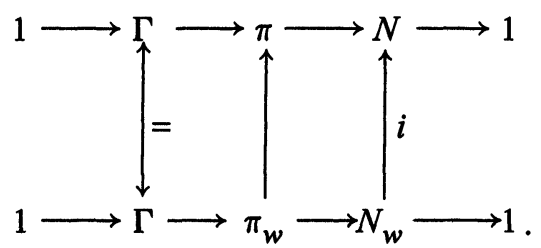

Of course $N_{w}$ must be finite and we may choose $\alpha \in N_{w}$ so that the cyclic group $\mathbf{Z} / q \mathbf{Z}$ generated by $\alpha$ is of prime order, $q$. Without loss of generality we may think of $N_{w} \simeq \mathbf{Z} / q \mathbf{Z}$. Then if $\pi_{w}$ has any elements of finite order other than $e$, they must map onto generators of $N_{w}$ since $\Gamma$ is torsion free. Thus $\pi_{w} \subset \pi$ contains a group, $H$, isomorphic to $\mathbf{Z} / q \mathbf{Z}$ and consequently on $\tilde{X} \times W=\tilde{P}$, the action of $H$ must leave an acyclic (cohomological) submanifold fixed. Thus the induced action on $X \times w$ of $N_{w}$ is not free. On the other hand, if $\pi_{w}$ is free the action of $N_{w}$ on $X \times w$ is free $[17,2.4]$. 
Therefore $\pi$ is torsion free if and only if the induced action of $N$ on $X \times W$ is a covering action. Of course, $\pi_{1}((X \times W) / N)$, in this case, is just that given by the extension $(*)$.

4.4. To put 4.3 into perspective algebraically we may consider any extension of the form $1 \rightarrow \Gamma \rightarrow \pi \rightarrow N \rightarrow 1$ where $\Gamma$ is the fundamental group of an aspherical manifold so that $\mathscr{Z}(\Gamma)=1$. Then for each finite subgroup $N_{1}$ of $N$ the induced extension $1 \rightarrow \Gamma \rightarrow \pi_{1} \rightarrow N_{1} \rightarrow 1$ is determined by $\left.\phi\right|_{N_{1}}: N_{1}$ $\rightarrow$ Out $\Gamma$. If $\left.\phi\right|_{N_{1}}$ is not an embedding, then $\pi_{1}$ and hence $\pi$ would have to contain elements of finite order. Thus for $\pi$ to be torsion free it is necessary that every finite subgroup of $N$ be mapped monomorphically by $\phi$ into Out $\Gamma$.

4.5. It is interesting to study the mapping $P=(X \times W) / N \stackrel{\mu}{\longrightarrow} W / N$. Let $w^{*}=\nu(w)$. Then $\mu^{-1}\left(w^{*}\right)=X / N_{w}$, whether or not the action on $X \times W$ is free. However, there is a definite regularity to this map, at least locally. If we take a slice $S_{w}$ at $w \in W$, then $N_{w}$ acts invariantly on $S_{w}$ so that $S_{w} / N_{w}$ $=W / N$ near the image $w^{*}=\nu(w)$. Further, the orbit map $X \times W \rightarrow X$ $\times W / N$ can be described locally near $x \times w$ to be $X \times S_{w} \rightarrow X_{N_{w}} \times S_{w}$. There are two induced projections which make the diagram commute:

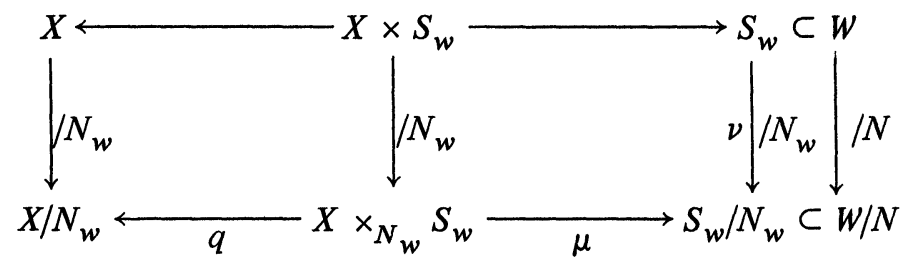

For a point $s^{*}=\nu(s), \mu^{-1}\left(s^{*}\right)=X /\left(N_{w}\right)_{s}$, and $q^{-1}\left(x^{*}\right)=S_{w} /\left(N_{w}\right)_{x}$. In particular, if $N_{w}$ acts freely on $X$ (if $\pi$ is torsion free) then $q$ is a fibering with fiber $S_{w}$ and structure group $N_{w}$. The map $\mu$ assigns to each orbit of $S_{w}$ the orbit of $X$ under the action of $\left(N_{w}\right)_{s}$, where $s$ lies on the particular orbit on $S_{w}$. Thus when $N$ acts as covering transformations on $X \times W$, the "fiber" $\mu^{-1}\left(w^{*}\right)$ is $X / N_{w}$ and a neighborhood of $\mu^{-1}\left(w^{*}\right)$ is a fiber bundle over $X / N_{w}$ with fiber a slice, $S_{w}$, of the action $N_{w}$ on $W$ at $w$ and with structure group $N_{w}$.

4.6. Our theorem is interesting only if we can show that there are $\Gamma$ for which hypothesis (ii) can be satisfied. We know of no examples for which hypotheses (i) and (ii) fail to be satisfied. Here are some examples for which the hypotheses can be verified.

1. Let $(W, N)=\left(\mathbf{R}^{1}, \mathbf{Z}\right)$. Then any aspherical manifold $X$ for which $\pi_{1}(X)=\Gamma, \mathscr{Z}(\Gamma)=1$, and $\Psi \circ i: \mathcal{H}(X) \rightarrow$ Out $\Gamma$ is surjective implies that all possible extensions $1 \rightarrow \Gamma \rightarrow \pi \rightarrow \mathbf{Z} \rightarrow 1$, must be the fundamental group of an aspherical manifold. For $\phi: \mathbf{Z} \rightarrow$ Out $\Gamma$ determines a congruence class of extensions. The group $\pi$ must be torsion free since both $\Gamma$ and $\mathbf{Z}$ are. The manifold $P=X \times_{\mathbf{Z}} \mathbf{R}^{1}$ is obtained by just taking the mapping torus of $\tilde{\phi}(1): X \rightarrow X$. For example, if $X$ is a closed 2-manifold $\neq S^{2}$ or $\mathbf{R} P_{2}$, any selfhomotopy equivalence can be deformed to a homeomorphism. Hence $\mathcal{H}(X)$ $\rightarrow$ Out $(\Gamma)$ is surjective.

We shall exhibit in 8.3 many more examples of aspherical $X$ where the homomorphism $\mathcal{H}(X) \rightarrow$ Out $(\Gamma)$ is onto.

2. In [3] we showed that almost all manifolds $X$ which are fibered over the 
circle with fiber a $k$-dimensional torus have the property $\pi_{1}(X)$ has trivial center and that the homomorphism $\mathfrak{H C}(X) \rightarrow$ Out $\Gamma$ is surjective and splits. Thus no matter which $(W, N)$ we may choose the extensions $1 \rightarrow \Gamma \rightarrow \pi \rightarrow N$ $\rightarrow 1$ all can be geometrically realized by our construction if and only if $\pi$ is torsion free.

As a specific illustration consider $X^{3}$ which is the mapping torus of the homeomorphism $h: T^{2} \rightarrow T^{2}$ defined by $h\left(z_{1}, z_{2}\right)=\left(z_{2}, z_{1} z_{2}\right)$. The group Out $(\Gamma) \simeq \mathbf{Z}_{2}$, and $\mathcal{H C}\left(X^{3}\right) \rightarrow \mathbf{Z}_{2} \rightarrow 1$ splits. Thus for any $\phi: N \rightarrow \mathbf{Z}_{2}$, if $N$ contains elements of finite order then $\pi$ cannot be torsion free. (We do not need that $N$ operates properly discontinuously on a contractible manifold.) Thus the only possibilities are when $N$ is torsion free. If, moreover, we have an action $(W, N)$ then our constructed $M(\pi)$ is of the form $X \times W / N$ or

$$
(X \times \mathbf{z} / 2 \mathbf{Z} W) /(\text { kernel } \phi),
$$

which is a fiber bundle over $W / N$ with fiber $X$ and structure group $\mathbf{Z} / 2 \mathbf{Z}$.

3. Let $X$ be a compact flat manifold with fundamental group $\Gamma$. Let $\operatorname{Aff}(X)$ be the group of diffeomorphisms of $X$ which preserve the Riemannian connection associated to $X$. The connected component of the identity, $\operatorname{Aff}_{0}(X)$ is isomorphic to a $k$-torus with $k$ equal to rank $\Gamma$. It is shown in [9] that $\mathrm{Aff}(X) / \operatorname{Aff}_{0}(X)$, under $\Psi \circ i$, is mapped isomorphically onto Out $\left(\pi_{1}\left(X, x_{0}\right)\right)$. We shall also obtain in $\S \S 8$ and 9 a proof of this theorem as a particular application of our general study of injective Seifert fiberings to be initiated in $\S 5$. Thus $\mathcal{H C}(X) \rightarrow$ Out $\pi_{1}\left(X, x_{0}\right)$ is surjective and, as we shall see in $\$ 9$, at least a portion of this epimorphism splits. Of course, there exist flat manifolds whose fundamental groups have trivial center. These groups are precisely the (torsion free) Bieberbach groups $\Gamma$ with $H^{1}(\Gamma ; \mathbf{Z})=0$.

This type of example will be generalized considerably for injective Seifert fiber spaces in $\$ 9$.

4.7. So far we have concentrated on groups, $\Gamma$, with trivial center. Let us now assume that $\Gamma$ has a nontrivial center $\mathscr{Z}(\Gamma)$. Form an extension $1 \rightarrow \Gamma$ $\rightarrow \pi \rightarrow L \rightarrow 1$, then $\mathscr{Z}(\Gamma)$ is a normal subgroup of $\pi$ since $\mathscr{Z}(\Gamma)$ is characteristic in $\Gamma$. Thus we have an extension $1 \rightarrow \mathscr{Z}(\Gamma) \rightarrow \pi \rightarrow N \rightarrow 1$ where the action of $N$ on $\mathscr{Z}(\Gamma)$ is given by conjugation by elements in $\pi$. Those elements of $\pi$ which are images of elements of $\Gamma$ all induce the identity on $\mathscr{Z}(\Gamma)$, thus the representation, $\phi: N \rightarrow$ Aut $\mathscr{Z}(\Gamma)$, factors through $\pi / \Gamma=L$.

Once again we run into Problem 3. (No examples are known where $\mathscr{Z}(\Gamma)$ is not finitely generated.) Let us therefore, for our purposes, consider those $\Gamma$ for which $\mathscr{Z}(\Gamma)$ is finitely generated. In trying to construct $\pi$ we would have specified the group $\pi / \Gamma$ and its action on Out $\Gamma$. This clearly leads to an extension

$$
1 \rightarrow \Gamma / \mathscr{Z}(\Gamma) \rightarrow \pi / \mathscr{Z}(\Gamma)=N \rightarrow \pi / \Gamma \rightarrow 1 .
$$

Therefore we lose no generality, as far as Problems 6 and 7 are concerned, in studying those extensions of a finitely generated torsion free abelian group, $\mathbf{Z}^{k}$, by a discrete group $N$. 
5. Seifert fiber spaces. The construction of aspherical manifolds: $\pi$ with normal abelian subgroups.

5.1. In this section we shall describe a special type of Seifert fibering. These are the geometric objects which will enable us to attack our problems for aspherical manifolds when $\pi$ has a finitely generated normal abelian subgroup. Our method applies to aspherical manifolds but does not depend essentially on them. Therefore we no longer restrict ourselves to aspherical manifolds but we shall not hesitate to illustrate and interpret our results in those terms. We introduce the term injective Seifert fiberings since it is this class of Seifert fiberings which are most relevant to the problems we have listed. Later, in this section, we shall briefly describe the more general class of Seifert fiberings studied in [14] because many of our results can be extended to general Seifert fiberings. Furthermore, we hope the success at hand may entice the reader to explore these general Seifert fiberings of [14] which arise quite naturally in, for example, differential geometry, algebraic geometry, complex manifolds, transformation groups, topology and number theory.

Once again we mention that we tacitly assume that all spaces $X$ are not pathological. In particular, they are nice enough to admit a viable covering space theory and occasionally the methods of algebraic topology.

5.2. INJECTIVE SEIFERT FIBER SPACES. Let $N$ be a discrete group and $\phi: N \rightarrow$ Aut $\left(\mathbf{Z}^{k}\right)$ be a homomorphism. Note that

$$
\text { Aut }\left(\mathbf{Z}^{k}\right)=\text { Out }\left(\mathbf{Z}^{k}\right)=\text { Aut }\left(\pi_{1}\left(T^{k}, 1\right)\right)=\operatorname{GL}(k, \mathbf{Z}) .
$$

Since GL $(k, \mathbf{Z}) \subseteq \mathrm{GL}(k, \mathbf{R})$ and preserves the integer lattice, every automorphism of the lattice gives rise to a continuous automorphism of $T^{k}$. In fact, Aut $\left(\mathbf{Z}^{k}\right)=$ Aut $\left(T^{k}, 1\right)$. Thus $\phi$ also is a representation of $N$ into the (linear) automorphisms of $T^{k}$.

By an injective Seifert fiber space $\left(T^{k}, X, \phi\right)$ we shall mean a path connected space $X$ for which:

1. There exists an epimorphism $\lambda: \pi_{1}\left(X, x_{0}\right) \rightarrow N \rightarrow 1$.

2. On the covering space $X^{\prime}$, corresponding to the kernel of $\lambda$, there exists a structure of a principal $k$-torus bundle which is compatible with the group of covering transformations $N$ on $X^{\prime}$. That is we require

$$
\left(t x^{\prime}\right) \alpha=\left(\phi\left(\alpha^{-1}\right)(t)\right)\left(x^{\prime} \alpha\right)
$$

for all $t \in T^{k}, x^{\prime} \in X^{\prime}, \alpha \in N$.

3. The quotient space $T^{k} \backslash X^{\prime}=W$ is simply connected.

4. The kernel of $\lambda$ is isomorphic to $\mathbf{Z}^{k}$.

Note the condition $\left(t x^{\prime}\right) \alpha=\left(\phi\left(\alpha^{-1}\right)\right)(t)\left(x^{\prime} \alpha\right)$ of 2 on $X^{\prime}$ really is equivalent to defining a right action of the semidirect product $T^{k} \circ N$ on $X^{\prime}$ by

$$
\left(x^{\prime}\right)(t, \alpha)=\left(t x^{\prime}\right) \alpha .
$$

The group law is

$$
(t, \alpha)(h, \beta)=(t \phi(\alpha)(h), \alpha \beta)
$$

which comes from 


$$
\begin{aligned}
\left(\left(x^{\prime}\right)(t, \alpha)\right) & (h, \beta)=\left(\left(t x^{\prime}\right) \alpha\right)(h, \beta) \\
& =\left(\phi\left(\alpha^{-1}\right)(t)\left(x^{\prime} \alpha\right)\right)(h, \beta)=\left(h\left(\left(\phi\left(\alpha^{-1}\right)(t)\right)\left(x^{\prime} \alpha\right)\right)\right) \beta \\
& =\left(\left(\phi\left(\alpha^{-1}\right)\right)(\phi(\alpha)(h))\left(\left(\phi\left(\alpha^{-1}\right)(t)\right)\left(x^{\prime} \alpha\right)\right)\right) \beta \\
& =\left(\phi\left(\alpha^{-1}\right)((\phi(\alpha)(h))(t))\left(x^{\prime} \alpha\right)\right) \beta=\left(t(\phi(\alpha)(h)) x^{\prime}\right)(\alpha \beta) .
\end{aligned}
$$

On $X^{\prime}$ we may factor out first the action of $T^{k}$. Put $W=T^{k} \backslash X^{\prime}$. Induced on $W$ is an action $(W, N)$ so that the corresponding natural maps:

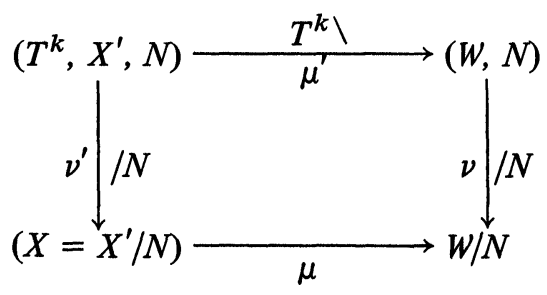

commute. We claim that the action $(W, N)$ must be properly discontinuous. Let $w \in W$. Then let $N_{w}$ be the stabilizer of $w$ and let $x^{\prime} \in X^{\prime}$ so that $\mu^{\prime}\left(x^{\prime}\right)=w$. The action of $N_{w}$ on $X^{\prime}$ leaves $\left(\mu^{\prime}\right)^{-1}(w)$ invariant. In fact, if $\nu^{\prime}$ is to be the orbit map of covering transformations the action of $N_{w}$ on the $T^{k}$ orbit through $x^{\prime}$ must be free. Thus we may characterize $\mu^{-1}(\nu(w))$ as $T^{k}\left(x^{\prime}\right) / N_{w}$, the orbit space of $T^{k}\left(x^{\prime}\right)$ under the free action.

It can be checked that the action $\left(T^{k}, N_{w}\right)$ is equivalent to an action determined by a crossed homomorphism $\chi: N_{w} \rightarrow T^{k}$ which, in turn, determines an element of $H_{\phi}^{1}\left(N_{w} ; T^{k}\right)$. Each crossed homomorphism induces an action given by $t * \alpha=\phi\left(\alpha^{-1}(t)\right) \chi\left(\alpha^{-1}\right)$. Cohomologous crossed homomorphisms yield equivalent actions and conversely. See [14, p. 133] for a very similar argument. The coboundary homomorphism $\delta: H_{\phi}^{1}\left(N_{w} ; T^{k}\right) \rightarrow H_{\phi}^{2}\left(N_{w} ; \mathbf{Z}^{k}\right)$ is an isomorphism. The element $\delta$ represents an extension $\pi_{w}$ of $\mathbf{Z}^{k}$ by $N_{w}$. This group $\pi_{w}$ is torsion free, if and only if, the action $\left(T^{k}, N_{w}\right)$ is free. See $[14, \S \S 3$ and 4$]$ and 5.3 for more details.

Let us call the mapping $\mu: X \rightarrow W / N$ arising from $\left(T^{k}, X, \phi\right)$ an injective Seifert fibering. We have seen that $W / N$ parametrizes both a class of immersions of the $k$-torus $T^{k}$ in $X$ and the family of group extensions corresponding to the fundamental group of the "fibers", $\mu^{-1}(\nu(w))$. In fact, $\mu^{-1}(\nu(w))$ is the quotient $T^{k} / N_{w}$ of the torus $T^{k}$ by a free action $\left(T^{k}, N_{w}\right)$ described by an explicit element $\chi \in H_{\phi}^{1}\left(N_{w} ; T^{k}\right)$. In turn, under the coboundary isomorphism $\delta \chi \in H_{\phi}^{2}\left(N_{w} ; \mathbf{Z}^{k}\right)$ defines an extension $1 \rightarrow \mathbf{Z}^{k} \rightarrow \pi_{w}$ $\rightarrow N_{w} \rightarrow 1$ which is the group extension given by the covering projection $T^{k} \rightarrow T^{k} / N_{w}$. Therefore each $T^{k} / N_{w}=\mu^{-1}(\nu(w))$ is a flat manifold and $\pi_{w}$ is a classical (torsion free) Bieberbach group.

5.3. Bieberbach ClasSES. As can be seen, Seifert fiberings, even of the injective type, are more general than fiber bundle projections since the topological type of the "fibers", $\mu^{-1}(\nu(w))$, may vary from point to point. To give a classification of Seifert fiberings over a space $Y$, say in the spirit of fiber bundles, one must carefully take into account all possible types of singularities. We do this now by investigating the converse of the construction we have just given. 
Let us begin with a discrete group $N$ and a homomorphism $\phi: N$ $\rightarrow \mathrm{GL}(k, \mathbf{Z})$. We choose a properly discontinuous (not necessarily free of course) action of $N$ on a simply connected space $W$ to be denoted by $(W, N)$. We are going to describe all possible injective Seifert fiberings $\mu: X \rightarrow W / N$. On the product space $T^{k} \times W=X^{\prime}$ we impose an action of $N$ on $X^{\prime}$ which is compatible with the obvious toral action. In fact, we seek $N$-actions so that

$$
\left(t x^{\prime}\right) \alpha=\left(\left(\phi\left(\alpha^{-1}\right)\right)(t)\right)\left(x^{\prime} \alpha\right) .
$$

In [11, §8], [12] and [14, §§3.9 and 4] we show that (the equivariant equivalence classes of) such $T^{k} \circ N$ actions are naturally in 1-1 correspondence with elements of the 2nd-cohomology group $H_{\phi}^{2}\left(N ; \mathbf{Z}^{k}\right)$. We shall describe this correspondence more carefully in $\$ 7$ where the mechanism is needed explicitly. For each $\left(X^{\prime}, T^{k} \circ N\right)$ we may now form $X=X^{\prime} / N$. The induced mapping $\mu: X \rightarrow W / N=\left(T^{k} \backslash X^{\prime}\right) / N$ is our desired Seifert fibering. There is but one flaw so far and that is the action $\left(X^{\prime}, N\right)$, while properly discontinuous, may not be free. If $\left(X^{\prime}, N\right)$ is not free, the mapping $\nu^{\prime}: X^{\prime} \rightarrow X^{\prime} / N$ is a regular branched covering map with nontrivial branching. While this offers no difficulties which cannot be easily overcome for building a theory (and corresponds to a modification of condition 2 in our definition of injective Seifert fibering), we need here only to single out those actions which give rise to (unbranched) covering transformations.

Let $B_{\phi}$ be the set of actions (or, equivalently, cohomology classes) for which the action of $N$ on $X^{\prime}$ is free. We call an element of $B_{\phi}$ a Bieberbach class and $B_{\phi}$ the set of Bieberbach classes. In general, $B_{\phi}$ is not a subgroup of $H_{\phi}^{2}\left(N ; \mathbf{Z}^{k}\right)$.

We shall now describe how one may characterize $B_{\phi}$ cohomologically. Let $a \in H_{\phi}^{2}\left(N ; \mathbf{Z}^{k}\right)$ determine our action $\left(X^{\prime}, N\right)$. For each $w \in W$, take the stabilizer $N_{w} \subseteq N$. $N_{w}$ is finite, since $(W, N)$ is properly discontinuous. On $\mu^{\prime-1}(w)=T^{k}$, there is induced an action $\left(T^{k}, N_{w}\right)$ from $\left(X^{\prime}, N\right)$. (The group $N_{w}$ leaves $\mu^{\prime-1}(w)$ invariant and any other element $\beta \in N$ sends $\mu^{\prime-1}(w)$ onto $\mu^{\prime-1}(w \cdot \beta)$.) Thus the stabilizer of $x^{\prime} \in T^{k}=\mu^{\prime-1}(w)$ is contained in $N_{w}$. In order that $N$ act freely on $X^{\prime}, N_{w}$ must act freely on $T^{k}$. This induced action corresponds to the cohomology class $i^{*}(a)$ induced from the homomorphism

$$
i^{*}: H_{\phi}^{2}\left(N ; \mathbf{Z}^{k}\right) \rightarrow H_{\phi}^{2}\left(N_{w} ; \mathbf{Z}^{k}\right)
$$

which arises from the inclusion $i: N_{w} \rightarrow N$. As mentioned earlier, the correspondence between actions and cohomology classes is natural. To each class in $H_{\phi}^{2}\left(N_{w} ; \mathbf{Z}^{k}\right)$ there corresponds the Baer equivalence class of extensions $1 \rightarrow \mathbf{Z}^{k} \rightarrow \pi \rightarrow N_{w} \rightarrow 1$. The action $\left(T^{k}, N_{w}\right)$ induces an action on the universal covering of $T^{k}$ by the extended group $\pi_{w}$ with $\mathbf{Z}^{k}$ corresponding to the group of covering transformations. Thus, $\pi_{w}=\pi_{1}\left(T^{k} / N_{w}\right)$ if the action $\left(T^{k}, N_{w}\right)$ is free. To be a free action is equivalent to $\pi_{w}$ being torsion free. Thus, the induced extension $\pi_{w}$ must be a classical Bieberbach group (a torsion free extension of $\mathbf{Z}^{k}$ by a finite group) whence our choice for the name of " $B_{\phi}$ ". To summarize our characterization of $B_{\phi}$ we have

5.4. Proposition $[14,3.1]$. The action $\left(X^{\prime}, N\right)$ is free, if and only if, the induced extension $i^{*}(a): 1 \rightarrow \mathbf{Z}^{k} \rightarrow \pi_{w} \rightarrow N_{w} \rightarrow 1$ is torsion free, for each $w \in W$.

Furthermore, for each Bieberbach class a (that is, when the action $\left(T^{k} \times W, N\right)$ 
is free) the group extension, as an element of $H_{\phi}^{2}\left(N ; \mathbf{Z}^{k}\right)$, is the same as the extension induced from the covering projection $\nu^{\prime}: X^{\prime} \rightarrow X$, namely, $1 \rightarrow \pi_{1}\left(T^{k} \times W\right) \rightarrow \pi_{1}(X) \rightarrow N \rightarrow 1$.

(We should remark that the condition 4, in conjunction with the first 3 conditions, is equivalent to the principal toral fiber bundle $X^{\prime} \rightarrow W$ being a trivial product bundle. If $a$ fails to be a Bieberbach class then $\pi$ maps onto $\pi_{1}(X)$ with nontrivial kernel and the regular covering $\nu^{\prime}$ will be branched.)

Thus to describe all the possible injective Seifert fiber spaces $\left(T^{k}, X, \phi\right)$ we

(i) take any properly discontinuous action $(W, N)$ of a discrete group $N$ on a simply connected space $W$;

(ii) form, on $T^{k} \times W$, all possible $T^{k} \circ N$ actions; these correspond to the elements of $H_{\phi}^{2}\left(N ; \mathbf{Z}^{k}\right)$;

(iii) find the subset $B_{\phi} \subset H_{\phi}^{2}\left(N ; \mathbf{Z}^{k}\right)$ of Bieberbach classes; these correspond to the $\left(T^{k} \times W, N\right)$ actions which are free.

The spaces $X=\left(T^{k} \times W\right) / N$ with the $N$ action satisfying (i), (ii), (iii) are all the possible injective Seifert fiber spaces satisfying 1, 2, 3, 4 of 5.2 with $\mu: X \rightarrow W / N$ the associated injective Seifert fibering.

5.5. ASPHERICAL MODELS. We may now characterize those Bieberbach classes that give rise to $K(\pi, 1)$ 's. Suppose $(W, N)$ is a properly discontinuous action and $W$ is contractible. Then for each Bieberbach class $a \in H_{\phi}^{2}\left(N ; \mathbf{Z}^{k}\right)$ corresponding to an extension $a: 1 \rightarrow \mathbf{Z}^{k} \rightarrow \pi \rightarrow N \rightarrow 1$ the space

$$
X=\left(T^{k} \times W\right) / N
$$

is a $K(\pi, 1)$ and the map $X \rightarrow W / N$ is a Seifert fibering. For example, suppose $W$ is contractible on which $N$ acts freely (as covering transformations), then $W / N$ is a $K(N, 1)$. Now each class $a \in H_{\phi}^{2}\left(N ; \mathbf{Z}^{k}\right)$ must be a Bieberbach class since $N_{w}=1$, for each $w \in W$. It is this particular construction that plays a crucial role in [30].

The example cited is not really immediately germane to our Problems 6 and 7. For, in general, $W$ will likely be infinite dimensional if $N$ is to act freely. Since we want finite dimensional $K(\pi, 1)$ 's (corresponding to groups $\pi$ with finite cohomological dimension) we assume that $W$ is a finite dimensional contractible space and that $N$ acts properly discontinuously on $W$. Since every $p$-subgroup of $N$ must fix some point of $W$, by the Smith theorems, one can easily prove that the extension $a: 1 \rightarrow \mathbf{Z}^{k} \rightarrow \pi \rightarrow N \rightarrow 1$ is torsion free, if and only if, $a \in H_{\phi}^{2}\left(N ; \mathbf{Z}^{k}\right)$ is a Bieberbach class $([14,3.9]$ and/or $[11,8.1])$.

We are led to the following important

THEOREM 6 ([11] AND [14]). Let $(W, N)$ be a properly discontinuous action of a discrete group of $N$ on a contractible manifold $W^{s}$ so that $W / N$ is compact. Then for each torsion free extension a: $1 \rightarrow \mathbf{Z}^{k} \rightarrow \pi \rightarrow N \rightarrow 1$ there exists a "model" aspherical manifold $M^{m}(\pi)$ and a Seifert fibering $\mu: M^{m}(\pi) \rightarrow W / N$. Furthermore $m=k+s$.

It is to be remarked that although the explicit models $M^{m}(\pi)$ appear to depend upon $W$ and the explicit action of $N$ the group $\pi$ does not. Any contractible manifold $W$ with any properly discontinuous action will suffice. Moreover, if $\pi$ is to be the fundamental group of a finite dimensional $K(\pi, 1)$ 
it must be torsion free. Thus, again, the weakest possible necessary condition on $\pi$ to be the fundamental group of an aspherical manifold is also a sufficient condition. Furthermore, our models for $M^{m}(\pi)$ assume a very nice form. For example, if $(W, N)$ is smooth, then $M^{m}(\pi)$ will be a smooth manifold and the immersions of $T^{k}$ will be smooth.

Unfortunately we do not know if each aspherical manifold $M^{m}(\pi)$ with $\pi$ containing a finitely generated normal abelian group has a model which arises this way. This is due, of course, to our not knowing whether the quotient group $\pi / \mathbf{Z}^{k}=N$ acts properly discontinuously on some contractible manifold $W$ so that the quotient space is compact. More generally let us pose

Problem 9. If $M^{m}$ is aspherical and $\Gamma$ is a normal subgroup of $\pi_{1}(M)$ so that $\Gamma$ is the fundamental group of some aspherical manifold $Y^{k}$, then does $\pi_{1}(M) / \Gamma=N$ act properly discontinuously on some contractible manifold $W^{m-k}$ so that $W / N$ is compact?

There seems to be no procedure for attacking this problem in general. However, there is certainly no shortage of examples of properly discontinuous actions on contractible manifolds. Moreover, if given a $(W, N)$ and one does find a Bieberbach class $a$ in $H_{\phi}^{2}\left(N ; \mathbf{Z}^{k}\right)$, then, in general, $B_{\phi}$ contains many more elements; see [11, 9.3] and [14, 3.3].

5.6. General SeIfert fiberings. The reader will have noticed that conditions $1-4$ of 5.2 are stronger than needed for much of the previous analysis to be carried out. To construct a workable theory of Seifert fiberings it only suffices to have 1 and

$2^{\prime}$. There exists a regularly branched covering (with $N$ as the group of regular branched covering transformations) on which the $k$-torus $T^{k}$ acts (effectively) so that $\left(t x^{\prime}\right) \alpha=\left(\phi\left(\alpha^{-1}\right)(t)\right)\left(x^{\prime} \alpha\right)$.

Once again this condition is equivalent to defining a right action of $T^{k} \circ N$ on $X^{\prime}$. We may form $X^{\prime} / N=X$ and still obtain a mapping $\mu: X \rightarrow W / N(W$ $\left.=X^{\prime} / T^{k}\right)$, with $\mu^{-1}(\nu(w))$ being the quotient space of the toral orbit $T^{k}\left(x^{\prime}\right)\left(=\mu^{\prime-1}(w)\right)$ by the induced action of the group $N_{w}$. There are really techniques sufficiently developed in [11] and [14] to systematically treat such fiberings. However, a great technical simplification occurs if we replace $2^{\prime}$ by $2^{\prime \prime} .2^{\prime \prime}=2^{\prime}$ with the added provisor that the toral action on $X^{\prime}$ be free. It is spaces $X$ which satisfy 1 and $2^{\prime \prime}$ that we called Seifert fiber spaces in [14]. The theory in [14] is completely worked out for what we call locally injective Seifert fiber spaces. This means that $\left(T^{k}, X, \phi\right)$ satisfies $1,2\left(=2^{\prime \prime}\right.$ and the regular covering transformations are unbranched) and 3. In particular, the theory becomes especially rich when one assumes that $(W, N)$ is a properly discontinuous group of holomorphic transformations. One obtains then what we called holomorphic Seifert fiber spaces.

As it turns out, condition 3 is simply a technical convenience and has no bearing whatsoever in a development of a theory. So we may assume condition 3 without any loss of generality.

Condition 4, on the other hand, is quite a different matter. In light of conditions (2) and (3),

Condition 4 is equivalent to the principal bundle $\left(T^{k}, X^{\prime}\right)$ being a product bundle, $\left(T^{k}, T^{k} \times W\right)$.

It is precisely the failure of $X^{\prime}$ to be a product bundle that causes 
complications in [14] and necessitates the use of sheaves for a complete analysis of the general setting of Seifert fiber spaces. (Also, in the complex analytic case condition 4 does not imply holomorphic triviality.)

To describe all the possible locally injective Seifert fiberings one need only replace the cohomology classes $H_{\phi}^{2}\left(N ; \mathbf{Z}^{k}\right)$ by the cohomology classes $H^{2}\left(N ; \mathscr{Z}^{k}\right)$ where $\mathscr{Z}^{k}$ is a sheaf associated to $(W, N)$ and $\phi$. The Bieberbach classes for locally injective actions are defined analogously as above. We refer the reader to [14] for details.

5.7. EXAMPLES. We have exhaustively treated many examples in our earlier papers. It may be of value to indicate now some of the more important sources for actions of a desired type $(W, N)$ and the Seifert fiber spaces associated to them

(a) A good way to create $(W, N)$ with $W$ a contractible manifold is to begin with an aspherical manifold $V$ and a finite group $F$ acting on $V$. This action on $V$ together with the covering transformations on the universal covering $\tilde{V}=W$ defines a properly discontinuous action $(W, N)$ of a group extension of $\pi_{1}(V)$ by $F, 1 \rightarrow \pi_{1}(V) \rightarrow N \rightarrow F \rightarrow 1$. This construction, as mentioned before, is explained in $[17, \S 2]$ and $[14,3.10]$. For example, if $F$ has a fixed point $v \in V$, then $N$ is isomorphic to a semidirect product $\pi_{1}(V, v) \circ F$. Each Bieberbach class $a \in H_{\phi}^{2}\left(N ; \mathbf{Z}^{k}\right)$ gives rise to an aspherical manifold $M$ $=\left(T^{k} \times W\right) / N$. The extension class determined by $a$ is $1 \rightarrow \mathbf{Z}^{k} \rightarrow \pi_{1}(M)$ $\rightarrow N \rightarrow 1$, and the normal subgroup $\mathbf{Z}^{k}$ is the image of the fundamental group of the covering space $\left(T^{k} \times W\right)$ of $M$.

How do we know that there are any torsion free extensions for some $k$ ? Let $1 \rightarrow \mathbf{Z}^{k} \rightarrow \pi_{F} \rightarrow F \rightarrow 1$ be any torsion free extension of $\mathbf{Z}^{k}$ by $F$. Such always exist for some $k$. Let the extension be denoted by $a \in H_{\theta}^{2}\left(F ; \mathbf{Z}^{k}\right)$, for some $\theta: F \rightarrow \mathrm{GL}(k, \mathbf{Z})$. If $\nu: N \rightarrow F$ denotes the epimorphism defining $N$ then $\nu^{*}(a)=b \in H_{\theta v}^{2}\left(N ; \mathbf{Z}^{k}\right)$. This extension must be torsion free since $\pi_{F}, \pi_{1}(V)$, and $\mathbf{Z}^{k}$ are torsion free.

The argument can be modified and $V$ replaced by any space $Y$. We still require that $\pi_{F}$ be torsion free but we do not need that $\pi_{1}\left(\left(T^{k} \times \tilde{Y}\right) / N\right)$ be torsion free. What is required is that for each extension $1 \rightarrow \mathbf{Z}^{k} \rightarrow \pi_{N_{\tilde{y}}} \rightarrow N_{\tilde{y}}$ $\rightarrow 1, \pi_{N_{\tilde{y}}}$ be torsion free. But under $\nu: N \rightarrow F, N_{\tilde{y}} \rightarrow F$ is a monomorphism where $\nu\left(N_{y}\right)=F_{y}$ is the stabilizer of $y \in Y$ and $\tilde{y}$ projects onto $y$. Since we require $\pi_{F}$ in $1 \rightarrow \mathbf{Z}^{k} \rightarrow \pi_{F} \rightarrow F \rightarrow 1$ to be torsion free, $\pi_{F_{y}}$ induced from $F_{y} \subset F$ is torsion free and consequently $\pi_{N_{j}}$ is also torsion free. Of course the extension

$$
1 \rightarrow \pi_{1}\left(T^{k} \times \tilde{Y}\right) \rightarrow \pi_{1}\left(\left(T^{k} \times \tilde{Y}\right) / N\right) \rightarrow N \rightarrow 1
$$

is still determined by the constructed Bieberbach class $\nu^{*}(a) \in H_{\theta \nu}^{2}\left(N ; \mathbf{Z}^{k}\right)$.

Other techniques for creating $(W, N)$ in a controlled way can be found in [16] and [15].

We point out that the closed flat manifolds are obtained by taking $V$ to be $a$ point.

(b) Toral actions. Let us observe that when $\phi: N \rightarrow \mathrm{GL}(k, \mathbf{Z})$ is the trivial homomorphism, then to any Bieberbach class in $B_{\phi}$ the associated Seifert fibering $\mu: X \rightarrow W / N$ is actually the orbit map of an induced toral action on $X$. 
(Recall that the semidirect product $T^{k} \circ N$ is $T^{k} \times N$ when and only when $\phi$ is trivial.) We may therefore ask which type of toral actions give rise to which type of Seifert fiberings?

Let $\left(T^{k}, X\right)$ be an arbitrary toral action. We define for each stability group, $T_{x}$, a canonical homomorphism $[11, \S 4]$

$$
\eta_{x}: T_{x} \rightarrow \pi_{1}(X, x) / \mathrm{im}\left(\mathrm{ev}_{*}^{x}\right)
$$

This is done as follows. If $t$ leaves $x$ fixed, choose any path $p(s)$ in $T$ with $p(0)=1, p(1)=t$. Then $p(s)(x)$ is a closed loop in $X$, based at $x$. In the quotient group $\pi_{1}(X, x) / \operatorname{im}\left(\operatorname{ev}_{*}^{x}\right)$ it represents $\eta_{x}(t)$. (Recall that im $\left(\operatorname{ev}_{*}^{y}\right)$ for any $y \in X$ is equal to im $\left(\mathrm{ev}_{*}^{x}\right)$ under any path isomorphism from $\pi_{1}(X, y)$ to $\pi_{1}(X, x)$.) By choosing any base point $x \in X$ we define

$$
N=\pi_{1}(X, x) / \operatorname{im}\left(\mathrm{ev}_{*}^{x}\right) .
$$

We say that the action $\left(T^{k}, X\right)$ is locally injective if the homomorphism $\eta_{x}$ is a monomorphism, for each $x \in X$. To be locally injective, each stability group $T_{x}$ must, of course, be finite. A more restrictive condition occurs where $\mathrm{ev}_{*}^{x}$ : $\pi_{1}\left(T_{1}^{k}\right) \rightarrow \pi_{1}(X, x)$ is a monomorphism, for some $x$ (and hence for all $x$ ) in $X$. If this condition holds we say the action $(T, X)$ is injective. Now for an arbitrary $\left(T^{k}, X\right)$ let $X^{\prime}$ denote the covering space associated with the central subgroup, image $\left(\mathrm{ev}_{*}^{x}\right)$. This action $\left(T^{k}, X\right)$ may be lifted to $\left(T^{k}, X^{\prime}\right)$ so as to commute with the covering transformations $N$ (see $[11, \S 4])$. Hence $N$ operates on the orbit space $W=T^{k} \backslash X^{\prime}$ and clearly $W / N$ may be identified with $X / T^{k}$ $=T^{k} \backslash X^{\prime} / N$. Hence the orbit mapping $\mu: X \rightarrow X / T^{k}$ is a Seifert mapping in the most general sense (but with $\phi$ trivial). The following answers the question posed above. See [11, 7.3], [12] and [14, 3.1].

THEOREM 7. The lifted action $\left(T^{k}, X^{\prime}\right)$ is free (respectively, $X^{\prime}$ splits into $T^{k} \times W$ and the action is the product action) and hence the Seifert fibering $\mu$ is locally injective (respectively, injective) if and only if the $\left(T^{k}, X\right)$ action is locally injective (respectively, injective).

The reader may wish to formulate the appropriate analogous conditions to $1,2^{\prime \prime \prime}$ and 3 for characterizing the nonlocally injective actions.

We have as an immediate corollary to Theorem 2 the

Proposition. If $\left(T^{k}, M\right)$ is an effective action on an aspherical manifold then the action is injective.

(c) Another example arising in algebraic geometry is the following. Suppose $M$ is a rational homology manifold and also a homological Kähler manifold. (For example $M$ could be the quotient of a nonsingular (complex) algebraic variety by a finite group of automorphisms.) Suppose $\left(T^{k}, M\right)$ is a continuous action with only finite isotropy subgroups. Then $\left(T^{k}, M\right)$ is homologically injective [14, $\S \S 7,8,10,12],[12],[6$, Chapter XII, §6]. (That is,

$$
\mathrm{ev}_{\#}^{x}: \pi_{1}\left(T^{k}, 1\right) \rightarrow \pi_{1}(M, x) \rightarrow H_{1}(M)
$$


is a monomorphism.) This is stronger than injective and is equivalent to each of the following:

(i) $a \in H^{2}\left(N ; \mathbf{Z}^{k}\right)$ is of finite order,

(ii) $M$ can be written as $T^{k} \times{ }_{F} Y$, that is, as an equivariant fiber bundle over $T^{k} / F$ with fiber $Y$ and finite structure group $F \subseteq T^{k}$.

Notice that this will tell us that any compact connected group of holomorphic automorphisms of a Kähler manifold must decompose the manifold as a holomorphic fibering over a torus. See also [8].

(d) This example is $(K / G, \Gamma)=(W, N)$ and was already mentioned in $\$ 2$. A special case is worth mentioning here. If $G$ is a connected nilpotent Lie group, then the center $C$ of $G$ is also a connected subgroup. Put the quotient group $C \backslash G=W$. If $\Gamma$ is a uniform discrete subgroup of $G$, then $\Gamma \cap C$ is the center of $\Gamma$ and on $C /(\Gamma \cap C) \times C \backslash G=G / \Gamma \cap C$, the group $\Gamma /(\Gamma \cap C)$ acts as a group of covering transformations. On the quotient nil-manifold, $G / \Gamma$, the torus group $C /(\Gamma \cap C)$ acts freely and injectively. We thank David Wigner for his help with this example.

(e) The classical 3-dimensional fiberings of Seifert [39] are all locally injective Seifert fiberings except for those that occur on the lens spaces, nonorientable handle and $\mathbf{R} P_{3} \# \mathbf{R} P_{3}$. If we assume the fundamental group is infinite and not $Z \circ \mathbf{Z}_{2}$ nor cyclic then they are all injective fiberings. However, our definition, even for injective Seifert fibering, is more general than Seifert's and we have, in dimension 3, injective Seifert fiberings which do not appear in Seifert's list.

(f) If $M$ is a closed manifold with nonpositive sectional curvature, then Lawson and Yau have shown [29] that the connected component of the group of isometries is a $k$-torus where $k=$ rank of the center of $\pi_{1}(M)$. Moreover, it can be seen that this action is homologically injective.

6. Computation of Out $\pi$. Our goal, which is purely group theoretic, is the computation of the automorphism groups and outer automorphism groups of the class of groups of the form

$$
1 \rightarrow \mathbf{Z}^{k} \stackrel{i}{\rightarrow} \pi \stackrel{j}{\rightarrow} N \rightarrow 1
$$

with

the subgroup $i\left(\mathbf{Z}^{k}\right)$ a characteristic subgroup of $\pi$.

The method of computation is related to our earlier procedures for the computation of Aut $\pi$ and Out $\pi$ where $\pi$ was of the form $\pi \simeq G \circ Z$ (see [17, $\S \S 4$ and 5]). These earlier computations led to our results on aspherical manifolds with no periodic maps. We shall digress for a moment to mention two common situations under which assumption (6.2) holds. In 6.14 we explain how one bypasses this assumption.

6.3. LeMma. In the extension (6.1), the subgroup $\mathbf{Z}^{k}$ is characteristic if either

(a) the extension is a central extension and $N$ is centerless, or

(b) $N$ has no nontrivial finitely generated normal abelian subgroups.

Proof of (b). Let $B \subset \pi$ be a finitely generated, normal, abelian subgroup. 
Then $j(B) \subset N$ is trivial which implies that $B \subset i\left(\mathbf{Z}^{k}\right)$. Thus, the image of $\mathbf{Z}^{k}$ is the unique, maximal, finitely generated, normal, abelian, subgroup of $\pi$.

6.4. An example of a type of group satisfying condition (b) is any discrete subgroup $N$ of a semisimple Lie group $G$, with trivial center, no compact factors and such that $G / N$ has finite volume. Professor David Wigner has kindly shown us the following proof of this assertion.

Let $G$ be a semisimple Lie group without compact factors. Let $N$ be a discrete subgroup such that $G / N$ has finite invariant measure. Let $A$ be an abelian normal subgroup of $N$. If $A$ acts on the Lie algebra $g$ of $G$ by conjugation (adjoint representation), then the elements of $A$ must have a common eigenvector $v$. Let $V$ be the linear span of all such common eigenvectors and let $v_{1}, \ldots, v_{k}$ be a basis of $V$ consisting of such eigenvectors. Then $v=\alpha_{1} v_{1}+\cdots+\alpha_{k} v_{k}$. If $a \in A$, let $a v_{i}=\beta_{i} v_{i}$. Then $a v$ $=\sum \alpha_{i} \beta_{i} v_{i}$ and $a v=\lambda v=\sum \alpha_{i} \lambda v_{i}$. Hence, $\alpha_{i} \lambda=\alpha_{i} \beta_{i}$, for all $i$, and consequently $\alpha_{i}=0$ or $\lambda=\beta_{i}$. Hence $v$ is a linear combination of those $v_{i}$ 's on which each $a \in A$ has the same eigenvalues as $v$. Let $V=V_{1} \oplus V_{2} \oplus \cdots \oplus V_{l}$ be a decomposition of $V$ into eigenspaces on which each element of $A$ acts by scalar multiplication. Each $V_{i}$ is generated by those $v_{i}$ 's on which the elements of $A$ act by multiplication by the same scalars.

Since $A$ is normal in $N, N$ permutes the $V_{i}^{\prime}$ s and some subgroup $N^{\prime}$ of finite index in $N$ stabilizes all $V_{i}$. Applying the density theorem of Borel to $N^{\prime}$ (which has finite measure since it is of finite index in $N$ ), $N^{\prime}$ has property $S$ by [36, Lemma 5.4, p. 79]. Moreover, the linear span of the matrices representing $N^{\prime}$ is equal to the linear span of $\mathrm{Ad} G$ in $\mathrm{g}$. Hence each $V_{i}$ is an ideal of $\mathrm{g}$ and $A$ acts by scalar multiplication on this ideal. Therefore $V$ has a $G$ invariant complement $W$ since $G$ is semisimple. If $W \neq 0$ then $A$ has a simultaneous eigenvector in $W$ which is a contradiction. Hence $W=0$ and $\mathfrak{g}=V$.

Now $A$ acts by scalar multiplication on the ideals $V_{i}$ which span g. Hence the adjoint action of $A$ commutes with the adjoint action of $G$ and $A \subset$ center of $G$, since $G /$ center $G$ is centerless.

6.5. To formulate the main result of this section we fix a group $N$, and a homomorphism $\phi: N \rightarrow$ Aut $\left(\mathbf{Z}^{k}\right)=\operatorname{GL}(k, \mathbf{Z})$. To any extension of the form (6.1) is associated a cohomology class $a \in H_{\phi}^{2}\left(N ; \mathbf{Z}^{k}\right)$. Since $\operatorname{GL}(k, \mathbf{Z})$ is the automorphism group of $\mathbf{Z}^{k}$, then by the action of $\operatorname{GL}(k, \mathbf{Z})$ on the coefficient group, we may assign to each matrix $g \in \mathrm{GL}(k, \mathbf{Z})$ an isomorphism $g_{*}$ : $H_{\phi}^{2}\left(N ; \mathbf{Z}^{k}\right) \rightarrow H_{g \phi g^{-1}}^{2}\left(N ; \mathbf{Z}^{k}\right)$. If $g \phi=\phi g$ then $g_{*}$ is an automorphism of $H_{\phi}^{2}\left(N ; \mathbf{Z}^{k}\right)$. Let $\Gamma_{1}^{\phi}(a) \subset \mathrm{GL}(k, \mathbf{Z})$ be the subgroup of all $g \in \mathrm{GL}(k, \mathbf{Z})$ so that

$$
\phi g=g \phi \text { and } g_{*}(a)=a .
$$

Let $\Gamma_{0}^{\phi}(a)$ be the subgroup of $\operatorname{GL}(k, \mathbf{Z})$ whose elements are of the form $\phi(\beta)$ for $\beta \in \mathscr{Z}(N)$, the center of $N$. We shall see that $\Gamma_{0}^{\phi}(a)$ is a normal subgroup of $\Gamma_{1}^{\phi}(a)$. Define $\Gamma^{\phi}(a)$ to be the quotient $\Gamma_{1}^{\phi}(a) / \Gamma_{0}^{\phi}(a)$.

We also know to each $\Phi \in$ Aut $N$ there is associated an isomorphism

$$
\Phi^{*}: H_{\phi}^{2}\left(N ; \mathbf{Z}^{k}\right) \rightarrow H_{\phi \circ \Phi}^{2}\left(N ; \mathbf{Z}^{k}\right) .
$$

We define a subgroup Aut $(a) \subset$ Aut $N$ by $\Phi \in$ Aut $(a)$ if and only if there is 
$g \in \mathrm{GL}(k, \mathbf{Z})$ with $g_{*}(a)=\Phi^{*}(a)$. In order to make sense we must have the compatibility relation on the operators:

$$
g \phi g^{-1}=\phi \circ \Phi \quad \text { or equivalently }(\phi \circ \Phi) g=g \phi .
$$

(This just says that $g: \mathbf{Z}^{k} \rightarrow \mathbf{Z}^{k}$ must be an $N$-module isomorphism where the action of $N$ on the image group is given by $\phi \circ \Phi$.) Since $\Phi^{*} \circ g_{*}=g_{*} \circ \Phi^{*}$, Aut $(a)$ is a subgroup of Aut $N$. (For, if

$$
\begin{array}{ll}
g_{1_{*}}(a)=\Phi_{1}^{*}(a), & g_{1} \phi g_{1}^{-1}=\phi \circ \Phi_{1}, \\
g_{2_{*}}(a)=\Phi_{2}^{*}(a), & g_{2} \phi g_{2}^{-1}=\phi \circ \Phi_{2},
\end{array}
$$

then

$$
\begin{aligned}
& \left(g_{2} \circ g_{1}\right) \phi(x)\left(g_{2} \circ g_{1}\right)^{-1}(\alpha)=g_{2}\left(g_{1}\left(\phi(x) g_{1}^{-1}\left(g_{2}^{-1}(\alpha)\right)\right)\right) \\
& =g_{2}\left(\phi\left(\Phi_{1}(x)\right)\left(g_{2}^{-1}(\alpha)\right)\right)=\phi\left(\Phi_{2} \circ \Phi_{1}\right)(x)(\alpha), \quad \alpha \in \mathbf{Z}^{k},
\end{aligned}
$$

and

$$
\begin{aligned}
\left(g_{2} \circ g_{1}\right)_{*}(a) & =\left(g_{2}\right)_{*} \circ \Phi_{1}^{*}(a)=\Phi_{1}^{*}\left(g_{2}(a)\right) \\
& \left.=\left(\Phi_{1}^{*} \circ \Phi_{2}^{*}\right)(a)=\left(\Phi_{2} \circ \Phi_{1}\right)^{*}(a) .\right)
\end{aligned}
$$

Since $\pi \rightarrow N$ is an epimorphism it follows that Inn $N$, the inner automorphisms of $N$, is a subgroup of Aut $(a)$. Hence we define Out $(a)$ $=\operatorname{Aut}(a) / \operatorname{Inn}(N)$ as a subgroup of Out $(N)$.

6.6. THEOREM 8. There are two short exact sequences:

$$
1 \rightarrow K \rightarrow \text { Out }(\pi) \rightarrow \text { Out }(a) \rightarrow 1,
$$

and

$$
1 \rightarrow \bar{H}_{\phi}^{1}\left(N ; \mathbf{Z}^{k}\right) \rightarrow K \rightarrow \Gamma^{\phi}(a) \rightarrow 1,
$$

where $\bar{H}_{\phi}^{1}\left(N ; \mathbf{Z}^{k}\right)$ is a well-defined quotient group of $H_{\phi}^{1}\left(N ; \mathbf{Z}^{k}\right)$. Furthermore, if either $N$ is centerless or if $a=0, \bar{H}_{\phi}^{1}$ is the full group $H_{\phi}^{1}\left(N ; \mathbf{Z}^{k}\right)$.

Proof. To aid in following the proof, which is somewhat complicated, we shall show there are two commutative diagrams:

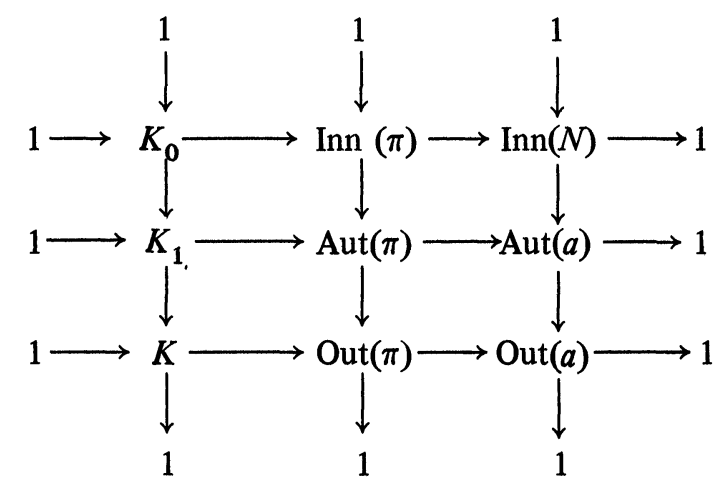


and,

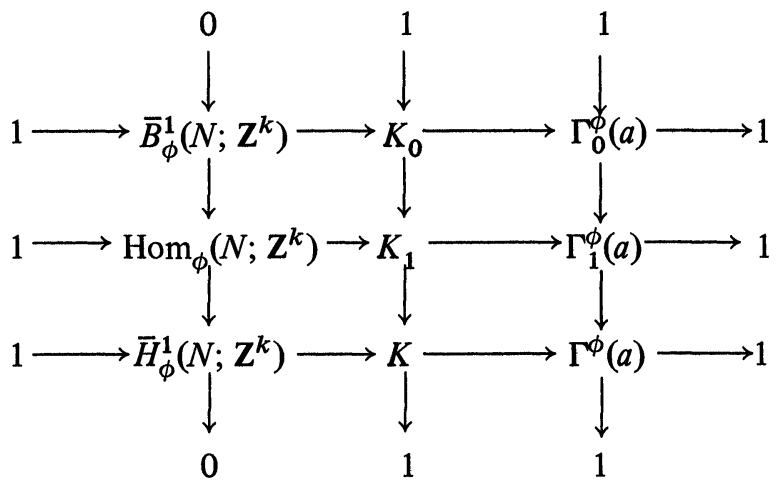

where all horizontal rows and vertical columns are exact. The bottom rows of each diagram will yield the theorem.

Let $f: N \times N \rightarrow \mathbf{Z}^{k}$ be a normalized cocycle representing $a$, then

$$
\pi=\mathbf{Z}^{k} \times N \text { with product }(n, \alpha)(m, \beta)=(n+\phi(\alpha) m+f(\alpha, \beta), \alpha \beta) .
$$

Recall that $\mathbf{Z}^{k}$ is assumed to be a characteristic subgroup. Thus, if $\sigma \in$ Aut $(\pi)$ is an automorphism of $\pi$, then we can write $\sigma(n, e)=(g(n), e)$ for some $g \in \mathrm{GL}(k, \mathbf{Z})$. Also, there exists a unique $\Phi \in \operatorname{Aut}(N)$ and a unique function $\lambda: N \rightarrow \mathbf{Z}^{k}$ so that $\sigma(0, \alpha)=(\lambda(\alpha), \Phi(\alpha))$. Since $(n, \alpha)=(n, e)(0, \alpha)$ we have

$$
\sigma(n, \alpha)=(g(n), e)(\lambda(\alpha), \Phi(\alpha))=(g(n)+\lambda(\alpha), \Phi(\alpha)) .
$$

Since $\sigma$ is an automorphism,

$$
\begin{aligned}
(\lambda(\alpha), \Phi(\alpha))(\lambda(\beta), \Phi(\beta))= & (\lambda(\alpha)+\phi(\Phi(\alpha))(\lambda(\beta)) \\
& +f(\Phi(\alpha), \Phi(\beta)), \Phi(\alpha) \Phi(\beta)) \\
= & (\sigma(0, \alpha))(\sigma(0, \beta))=\sigma(f(\alpha, \beta), \alpha \beta) \\
= & (g(f(\alpha, \beta))+\lambda(\alpha \beta), \Phi(\alpha) \Phi(\beta)) .
\end{aligned}
$$

Hence we obtain an identity

$$
g(f(\alpha, \beta))-f(\Phi(\alpha), \Phi(\beta))=\lambda(\alpha)-\lambda(\alpha \beta)+\phi(\Phi(\alpha))(\lambda(\beta)),
$$

from which we may conclude that $g_{*}(a)=\Phi^{*}(a)$, as an element

$$
H_{\phi \circ \Phi}^{2}\left(N ; \mathbf{Z}^{k}\right) \text {. }
$$

Thus an automorphism yields a triple $(g, \lambda, \Phi)$ for which (6.7) holds.

Conversely, given any triple $(g, \lambda, \Phi)$ for which $g \phi g^{-1}=\phi \circ \Phi$ and (6.7) holds define an automorphism $\sigma \in$ Aut $(\pi)$ by

$$
(n, \alpha) \rightarrow(g(n)+\lambda(\alpha), \Phi(\alpha)) .
$$

Recall Aut $(a) \subset$ Aut $(N)$ are those automorphisms $\Phi$ of $N$ so that there 
exists $g \in \mathrm{GL}(k, \mathbf{Z})$ with $g_{*}(a)=\Phi^{*}(a)$. Since this condition implies that (6.7) holds, for some choice $\lambda: N \rightarrow \mathbf{Z}^{k}$, we have a short exact sequence $1 \rightarrow K_{1} \rightarrow$ Aut $(\pi) \rightarrow$ Aut $(a) \rightarrow 1$. Note that Inn $(N) \subset$ Aut $(a)$. Now let $K_{0}$ be the subgroup of Inn $\pi$ which maps onto the trivial element in Aut $(a)$. This subgroup is normal in $K_{1}$, and in Inn $\pi$. Let $K=K_{1} / K_{0}$. Then we get the short exact sequence

$$
1 \rightarrow K \rightarrow \text { Out }(\pi) \rightarrow \text { Out }(a) \rightarrow 1,
$$

where Out $(a)=$ Aut $(a) / \operatorname{Inn}(N)$.

The group $K_{1}$ may be regarded as the group of all pairs $(g, \lambda$, id $)=(g, \lambda)$ where $\lambda: N \rightarrow \mathbf{Z}^{k}$ satisfies

$$
g(f(\alpha, \beta))-f(\alpha, \beta)=\lambda(\alpha)-\lambda(\alpha \beta)+\phi(\alpha) \lambda(\beta) .
$$

The product rule is

$$
(g, \lambda)\left(g_{1}, \lambda_{1}\right)=\left(g g_{1}, g \circ \lambda_{1}+\lambda\right) .
$$

Recall that $\Gamma_{1}^{\phi}(a)=\left\{g \mid g \phi=\phi g\right.$ and $\left.g_{*}(a)=a\right\}$. Therefore, there exists $\lambda: N \rightarrow \mathbf{Z}^{k}$ so that (6.7) holds with $\Phi=$ id. Consequently, there is an automorphism $(g, \lambda$ id $)=(g, \lambda)$, hence an epimorphism $K_{1} \rightarrow \Gamma_{1}^{\phi}(a)$. The kernel is the subgroup of pairs $(I, \lambda)$. For such a pair

$$
0=\lambda(\alpha)-\lambda(\alpha \beta)+\phi(\alpha) \lambda(\beta) .
$$

That is, $\lambda \in \operatorname{Hom}_{\phi}\left(N, \mathbf{Z}^{k}\right)$, the group of crossed homomorphisms. Hence we have shown that the sequence $1 \rightarrow \operatorname{Hom}_{\phi}\left(N, \mathbf{Z}^{k}\right) \rightarrow K_{1} \rightarrow \Gamma_{1}^{\phi}(a) \rightarrow 1$ is exact.

6.8. INNER AUTOMORPHISMS OF $\pi$. We shall characterize those automorphisms in $K_{0}$. To be in $K_{0}$ the automorphism $(g, \lambda)$ must be an inner automorphism of $\pi$. That is $(g, \lambda)(n, \alpha)=(g(n)+\lambda(\alpha), \alpha)$ must be equal to $(m, \beta)\left((n, \alpha)(m, \beta)^{-1}\right)$ for all $(n, \alpha)$ and some $(m, \beta)$. Now

$$
(m, \beta)^{-1}=\left(-f\left(\beta^{-1}, \beta\right)-\phi\left(\beta^{-1}\right)(m), \beta^{-1}\right),
$$

so for an arbitrary inner automorphism we have

$$
\begin{array}{r}
(m, \beta)\left(n+\phi(\alpha)\left(-f\left(\beta^{-1}, \beta\right)-\phi\left(\beta^{-1}\right)(m)\right)+f\left(\alpha, \beta^{-1}\right), \alpha \beta^{-1}\right) \\
=\left(m+\phi(\beta)\left[n+\phi(\alpha)\left(-f\left(\beta^{-1}, \beta\right)-\phi\left(\beta^{-1}\right)(m)\right)+f\left(\alpha, \beta^{-1}\right)\right]\right. \\
\left.+f\left(\beta, \alpha \beta^{-1}\right), \beta \alpha \beta^{-1}\right) .
\end{array}
$$

If we put $\alpha=e$, then we have

$$
\left(m+\phi(\beta)(n)-\phi(\beta) f\left(\beta^{-1}, \beta\right)-m+f\left(\beta, \beta^{-1}\right), e\right) .
$$

But from the general transitivity relation $[32$, p. $111,(4.5)]$,

$$
\phi(x) f(y, z)+f(x, y z)=f(x, y)+f(x y, z),
$$

we have that $-\phi(\beta) f\left(\beta^{-1}, \beta\right)+f\left(\beta, \beta^{-1}\right)=0$. Consequently, for conjugation by $(m, \beta), g=\phi(\beta)$, and $\Phi$ is conjugation by $\beta$.

Let us now consider $(n, \alpha)=(0, \alpha)$. We obtain 


$$
\begin{array}{r}
(\lambda(\alpha), \Phi(\alpha))=\left(m+\phi(\beta \alpha)\left(-f\left(\beta^{-1}, \beta\right)-\phi\left(\beta^{-1}\right)(m)\right)\right. \\
\left.+\phi(\beta) f\left(\alpha, \beta^{-1}\right)+f\left(\beta, \alpha \beta^{-1}\right), \beta \alpha \beta^{-1}\right) \\
=\left(m-\phi\left(\beta \alpha \beta^{-1}\right)(m)+\phi(\beta \alpha)\left(-f\left(\beta^{-1}, \beta\right)\right)\right. \\
\left.+f(\beta, \alpha)+f\left(\beta \alpha, \beta^{-1}\right), \beta \alpha \beta^{-1}\right) .
\end{array}
$$

This comes from

$$
\phi(\beta) f\left(\alpha, \beta^{-1}\right)=f(\beta, \alpha)+f\left(\beta \alpha, \beta^{-1}\right)-f\left(\beta, \alpha \beta^{-1}\right) .
$$

We may collect terms further and obtain

$$
m-\phi\left(\beta \alpha \beta^{-1}\right)(m)+f(\beta, \alpha)-f\left(\beta \alpha \beta^{-1}, \beta\right)=\lambda(\alpha) .
$$

In summary, if $(g, \lambda, \Phi)$, in $\operatorname{Inn}(\pi)$, represents conjugation by $(m, \beta)$, then $(g, \lambda(\alpha), \Phi)$ is

$$
\left(\phi(\beta), m-\phi\left(\beta \alpha \beta^{-1}\right)(m)+f(\beta, \alpha)-f\left(\beta \alpha \beta^{-1}, \beta\right), \beta \alpha \beta^{-1}\right) .
$$

In order for $(g, \lambda, \Phi)$ to be in $K_{0}$, then $\beta \alpha \beta^{-1}=\alpha$, for all $\alpha$, hence $\beta \in \mathscr{Z}(N)$. Then $\lambda(\alpha)$ simplifies to

$$
\lambda(\alpha)=m-\phi(\alpha)(m)+f(\beta, \alpha)-f(\alpha, \beta) .
$$

Recall that $\Gamma_{0}^{\phi}(a)=\{g \mid g \in \mathrm{GL}(k, \mathbf{Z})$ so that $g=\phi(\beta)$, for some $\beta$ $\in \mathscr{Z}(N)\}$. Certainly under $K_{1} \rightarrow \Gamma_{1}^{\phi}(a), K_{0}$ is carried into $\Gamma_{0}^{\phi}(a)$. We claim the image is also $\Gamma_{0}^{\phi}(a)$. If we choose $\beta \in \mathscr{Z}(N)$ arbitrarily, then conjugation by $(m, \beta)$, for some $m \in \mathbf{Z}^{k}$, yields $\left(\phi(\beta), \lambda_{\beta}\right.$, id), an element in $K_{0}$, whose image in $\Gamma_{0}^{\phi}(a)$ is $\phi(\beta)$. Thus, the image of $K_{0}$ is $\Gamma_{0}^{\phi}(a)$.

Let us check whether or not $\lambda_{\beta}$ is a crossed homomorphism.

$$
\begin{aligned}
\lambda_{\beta}(\alpha)-\lambda_{\beta}(\alpha \gamma)+\phi(\alpha) \lambda_{\beta}(\gamma)= & f(\beta, \alpha)-f(\alpha, \beta)-f(\beta, \alpha \gamma)+f(\alpha \gamma, \beta) \\
& +\phi(\alpha) f(\beta, \gamma)-\phi(\alpha) f(\gamma, \beta) .
\end{aligned}
$$

Since,

$$
\phi(\alpha) f(\beta, \gamma)-f(\alpha, \beta)=f(\alpha \beta, \gamma)-f(\alpha, \beta \gamma),
$$

and

$$
f(\alpha \gamma, \beta)-\phi(\alpha) f(\gamma, \beta)=f(\alpha, \beta \gamma)-f(\alpha, \gamma),
$$

we have the expression equal to

$$
\begin{aligned}
f(\beta, \alpha)+f(\alpha \beta, \gamma)-f & (\alpha, \beta \gamma)-f(\beta, \alpha \gamma)+f(\alpha, \beta \gamma)-f(\alpha, \gamma) \\
& =f(\beta, \alpha)+(f(\beta \alpha, \gamma)-f(\beta, \alpha \gamma))-f(\alpha, \gamma) \\
& =f(\beta, \alpha)+(\phi(\beta) f(\alpha, \gamma)-f(\beta, \alpha))-f(\alpha, \gamma) \\
& =\phi(\beta) f(\alpha, \gamma)-f(\alpha, \gamma)=\lambda_{\beta}(\alpha)-\lambda_{\beta}(\alpha \gamma)+\phi(\alpha) \lambda_{\beta}(\gamma) .
\end{aligned}
$$

Now $\lambda_{\beta}$ will be a crossed homomorphism if 
(a) $\phi(\beta)$ is trivial for $\beta \in \mathscr{Z}(N)$ or, more generally,

(b) $\phi(\beta) f(\alpha, \gamma)-f(\alpha, \gamma)=0$, for all $\alpha, \gamma \in N$.

Let us point out that if $\phi$ is trivial on $\mathscr{Z}(N)$ then every element of $K_{0}$ is a crossed homomorphism and $\Gamma_{0}^{\phi}(a)$ is trivial. If, on the other hand, we were to assume $f$ is trivial, then the elements of $K_{0}$ are pairs $(g, \lambda)$, where $g$ $=\phi(\beta)$ and $\lambda(\alpha)=m-\phi(\alpha) m$, a principal crossed homomorphism. $K_{0}$ then maps onto $\phi(\mathscr{Z}(N))$ with kernel the set of principal crossed homomorphisms $B^{1}\left(N ; \mathbf{Z}^{k}\right)$.

In general, $K_{0}$ is the group of inner automorphisms of $\pi$ which induce the identity on $N . K_{0}$ also maps onto $\Gamma_{0}^{\phi}=\phi(\mathscr{Z}(N))$ with kernel the group of inner automorphisms of $\pi$ which induce the identity on both $\mathbf{Z}^{k}$ and $N$. As such this kernel, $\bar{B}_{\phi}^{1}\left(N ; \mathbf{Z}^{k}\right)$, consists of the crossed homomorphisms corresponding to conjugation by elements $(m, \beta)$ with $\beta \in \mathscr{Z}(N)$ and $\phi(\beta)$ trivial. $\bar{B}_{\phi}^{1}\left(N ; \mathbf{Z}^{k}\right)$ contains all principal crossed homomorphisms, $B_{\phi}^{1}\left(N ; \mathbf{Z}^{k}\right)$, corresponding to conjugation by all elements of the form $(m, e)$. This group $B_{\phi}^{l}\left(N ; \mathbf{Z}^{k}\right)$ is naturally isomorphic to $\mathbf{Z}^{k} / \mathbf{Z}^{k} \cap \mathscr{Z}(\pi)$. Now $K_{1}$ is the group of automorphisms of $\pi$ which induce the trivial automorphism on $N$. It has a normal subgroup (the kernel of $K_{1} \rightarrow \Gamma_{1}^{\phi}(a)$ ) $\operatorname{Hom}_{\phi}\left(N ; \mathbf{Z}^{k}\right.$ ), the group of crossed homomorphisms, which as automorphisms induce the identity on $\mathbf{Z}^{k}$ and on $N$. We define

$$
\bar{H}_{\phi}^{1}\left(N ; \mathbf{Z}^{k}\right)=\operatorname{Hom}_{\phi}\left(N ; \mathbf{Z}^{k}\right) / \bar{B}_{\phi}^{1}\left(N ; \mathbf{Z}^{k}\right) .
$$

Of course we have the following exact sequence:

$$
0 \rightarrow \bar{B}_{\phi}^{1}\left(N ; \mathbf{Z}^{k}\right) / B_{\phi}^{1}\left(N ; \mathbf{Z}^{k}\right) \rightarrow H_{\phi}^{1}\left(N ; \mathbf{Z}^{k}\right) \rightarrow \bar{H}_{\phi}^{1}\left(N ; \mathbf{Z}^{k}\right) \rightarrow 0 .
$$

$\bar{H}^{1}$ is to be interpreted as the group of outer automorphisms of $\pi$ which induces both the identity on $\mathbf{Z}^{k}$ and on $N$. It is a normal subgroup of $K$, those outer automorphisms of $\pi$ which induce the identity on $N$. We may interpret $H^{1}\left(N ; \mathbf{Z}^{k}\right)$ as the group of automorphisms of $\pi$ which induce the identity on $\mathbf{Z}^{k}$ and $N$ modulo those inner automorphisms arising from conjugation by elements of $\mathbf{Z}^{k}$.

We have obtained the commutative diagrams mentioned just after the statement of the theorems and have checked vertical exactness and horizontal exactness everywhere except for the last line of the second diagram. This last sequence is exact by either diagram chasing or direct examination. This completes the proof of Theorem 8 .

6.9. Computations AND EXAMPLES. Let us try to get a feeling for the two exact sequences of the theorem. We begin with

$$
\Gamma^{\phi}(a)=(\text { centralizer of } \phi(N)) \cap\left\{g \mid g_{*}(a)=a\right\} / \phi(\mathscr{Z}(N)) .
$$

(i) $a=0$. Then (6.1) is congruent to a semidirect product and $f$ can be taken to be 0 . Now any pair $(g, \Phi)$ for which $g \phi=\phi \circ \Phi g$ implies that $g_{*}(a)$ $=\Phi^{*}(a)=0$. Hence one can form the triple $(g, \Phi, \lambda)$ for some function $\lambda$ satisfying (6.7) and obtain an automorphism of $\pi$. Therefore,

$$
\Gamma^{\phi}(a)=\text { centralizer of } \phi(N) \text { in GL }(k, \mathbf{Z}) / \phi(\mathscr{Z}(N)) .
$$

Similarly, 


$$
\text { Aut }(a)=\left\{\Phi \mid \text { there exists } g \text { so that } g \phi g^{-1}=\phi \circ \Phi\right\}
$$

and,

$$
\text { Out }(a)=\operatorname{Aut}(a) / \operatorname{Inn}(N) \text {. }
$$

Note also, since $f$ is trivial, $\bar{B}_{\phi}^{1}=B_{\phi}^{1}$ and $\bar{H}_{\phi}^{1}\left(N ; \mathbf{Z}^{k}\right)=H^{1}\left(N ; \mathbf{Z}^{k}\right)$.

(ii) $\phi(\mathscr{Z}(N))=I$. Then $\Gamma_{0}^{\phi}(a)=0$ and $\Gamma_{1}^{\phi}(a)=\Gamma^{\phi}(a)$, and $K_{0}=\bar{B}_{\phi}^{1}$. If, in addition, $N$ is centerless, then $\bar{B}_{\phi}^{1}=B_{\phi}^{1}$ and $\bar{H}^{1}=H^{1}$.

(iii) $N$ is abelian. Then $\operatorname{Inn}(N)=0$, Aut $(a)=$ Out $(a)$, and $\Gamma_{0}^{\phi}(a)$ $=\phi(N)$.

(iv) $N$ is $\mathbf{Z}$. This is an important example. We may obtain the results of [17, $\S 6]$ and the results for the fundamental groups of the manifolds in [17, §8.2]. First we point out that $a=0=H_{\phi}^{2}\left(\mathbf{Z} ; \mathbf{Z}^{k}\right)$, and consequently we may take $f=0$, and $\pi=\mathbf{Z}^{k} \circ_{\phi} \mathbf{Z}$. Also we may use the results of (i) and (iii).

The automorphism $\phi$ is completely determined by its value

$$
\phi(1) \in \mathrm{GL}(k, \mathbf{Z}) .
$$

Now, $\mathbf{Z}^{k}$ is characteristic in $\pi$ if it is the kernel of $\pi \rightarrow \pi /[\pi, \pi] \otimes \mathbf{Q}$. It is shown in $[17,4.1]$ that this is actually the case if $\operatorname{det}(I-\phi(1)) \neq 0$.

Actually when we are dealing with $a=0$, that is $\pi=\mathbf{Z}^{k} \circ N$, one can show in a similar manner that $\mathbf{Z}^{k}$ is characteristic if

$$
H_{0}^{\phi}\left(N ; H_{1}\left(\mathbf{Z}^{k} ; \mathbf{Q}\right)\right) \simeq H_{0}^{\phi}\left(N ; \mathbf{Q}^{k}\right)=0 .
$$

This would be implied if there exists some $\alpha \in N$ so that $\operatorname{det}(I-\phi(\alpha)) \neq 0$. A sequence is that $H_{1}(\pi ; \mathbf{Q})$ (trivial action of $\pi$ on $\mathbf{Q}$ ) is isomorphic to $H_{1}(N ; \mathbf{Q})$.

Now Aut $(a)=$ Out $(a) \subset$ Aut $(\mathbf{Z}) \simeq \mathbf{Z}_{2}$, which is, in terms of the compatibility relations,

$$
g \phi(1) g^{-1}=\phi(1) \quad \text { or } \quad g \phi(1) g^{-1}=(\phi(1))^{-1} .
$$

Consequently, we have the two exact sequences:

$$
\begin{aligned}
& 0 \rightarrow H_{\phi}^{1}\left(\mathbf{Z} ; \mathbf{Z}^{k}\right) \rightarrow K \rightrightarrows C(\phi(1)) / \phi(\mathbf{Z}) \rightarrow 1, \\
& 1 \rightarrow K \rightarrow \text { Out }(\pi) \rightarrow \text { Out }(a)=\{0\} \text { or } \mathbf{Z}_{2} \rightarrow 1 .
\end{aligned}
$$

Here $C(\phi(1))$ denotes the centralizer of $\phi(1)$ in GL $(k, \mathbf{Z})$. (Compare with [17, 4.5 and 4.6]. It remains to check the asserted splitting. Now an element in $K_{1}$ can be represented by $\left(g, \lambda\right.$, id). For each $g \in \Gamma_{1}^{\phi}(a)$, there is a $\lambda$ so that $(g, \lambda$, id $) \in K_{1}$. Now if $a=0$ and we take $f=0$, then $\lambda$ is a crossed homomorphism. Consequently, $g \rightarrow(g, 0, \mathrm{id})$ is a splitting homomorphism. Note that only $a=0$ is used for this argument and not that $N=\mathbf{Z}$.

The reader might wish to examine some very specific computations for Out $\left(Z^{k}{ }_{\phi} Z\right)$. Many computations can be found in $[17, \S 6]$ and $[18, \S 2]$ where the chief interest lay in getting Torsion (Out $(\pi)$ ) as small as possible and $\pi$ centerless.

In computing Out $\pi$ from Theorem 8 the difficult parts are 
Out $(a)$ and $\Gamma^{\phi}(a)$. For each $\alpha \in N, \phi(\alpha)$ generates a cyclic group in GL $(k, \mathbf{Z})$, and $\Gamma_{1}^{\phi}(a)$ is the intersection of all the centralizers $g$ of $\phi(\beta)$, for all $\beta \in N$, and such that $g_{*}(a)=a$. We shall sketch the method employed in [17], [18] and [13] which enables one to closely estimate Out $\pi$ for $\pi=\mathbf{Z}^{k} \circ \mathbf{Z}$.

Let $\phi(1) \in \mathrm{GL}(k, \mathbf{Z})$, and $f(x)=\operatorname{det}(I x-\phi(1))$ be its characteristic polynomial with $\lambda_{1}, \ldots, \lambda_{k}$ its roots.

6.11. Corollary [18]. If

(1) $\lambda_{1}, \ldots, \lambda_{k}$ are all distinct and $\neq \pm 1$,

(2) $f(1) \neq 0$,

(3) $f(x) \not \equiv x^{k} f(1 / x) / f(0)$, then,

$$
\text { Out } \pi \simeq\left(\mathbf{Z}^{k} /(I-\phi(1)) \mathbf{Z}^{k}\right) \circ(C(\phi(1)) / \phi(\mathbf{Z}))
$$

where $H_{\phi}^{1}\left(\mathbf{Z}, \mathbf{Z}^{k}\right) \simeq\left(\mathbf{Z}^{k} /(I-\phi(1)) \mathbf{Z}^{k}\right)$ is a finite abelian group with order equal to $|f(1)|$, and $C(\phi(1)) /(\phi(\mathbf{Z}))$ is a finitely generated abelian group. Furthermore, if

(4) no $\lambda_{1}, \ldots, \lambda_{k}$ is a complex root of unity, then, $\pi=\mathbf{Z}^{k} \circ \mathbf{Z}$ is centerless.

Moreover, if

(5) $f(x)$ is irreducible over $\mathbf{Q}$, then, $C(\phi(1))$ is a subgroup of $\mathbf{Z}^{r} \times F$, where $r$ is one less than the sum of the real zeros and one half the complex zeros of $f(x)$ and $F$ is a finite group $(F= \pm I$, if $k$ is odd or $f(0)<0)$, and if

(6) $f\left( \pm x^{n}\right)$ is irreducible, all $n>1$, then, $\phi(\mathbf{Z})$ is a direct summand of $C(\phi(1))$.

Proof. It is well known that $f(1)=\operatorname{det}(I-\phi(1))$. If $f(1) \neq 0, \mathbf{Z}^{k}$ is characterized by (iv) and $(I-\phi(1)): \mathbf{Z}^{k} \rightarrow \mathbf{Z}^{k}$ is a monomorphism with cokernel $H_{0}^{\phi}\left(\mathbf{Z} ; \mathbf{Z}^{k}\right)$, a finite group whose order is $|f(1)|$.

Now Aut $(a) \subset \mathbf{Z}_{2}$ and is actually 0 because there is no matrix $g$ $\in \mathrm{GL}(k, \mathbf{Z})$ so that $(\phi(1))^{-1}=g \phi(1) g^{-1}$. For, the right-hand side of (3) is the characteristic polynomial of $(\phi(1))^{-1}$ and the characteristic polynomial of $g \phi g^{-1}$ is still $f(x)$. So no possible solutions exist.

It only remains to compute $\Gamma^{\phi}(a)$. We need to compute $C(\phi(1))$ and understand how $\phi(\mathbf{Z})$ imbeds in $C(\phi(1))$. From linear algebra we know, since all eigenvalues are distinct, that all rational $k \times k$ matrices which commute with $\phi(1)$ are the polynomials in $\phi(1)$ with rational coefficients. The intersection with the matrices in GL $(k, \mathbf{Z})$ gives a finitely generated abelian group.

Condition 5 also tells us that the commuting rational matrices may be identified with the finite extension field $\mathbf{Q}(\lambda)$, for any root $\lambda$ of $f(x) . C(\phi(1))$ then becomes $\mathrm{GL}(k, \mathbf{Z}) \cap \mathbf{Q}(\lambda)$ and is a subgroup of the group of units of the algebraic integers in $\mathbf{Q}(\lambda)$. This group is isomorphic, by Dirichlet's unit theorem, to $\mathbf{Z}^{r} \oplus F$, where $F$ are the roots of unity in $\mathbf{Q}(\lambda)$. Condition 6 implies that there is no matrix $g$ so that $\phi(1)= \pm g^{n}$, for all $n>1$, and so $\phi(1)$ would have to be a direct summand of $C(\phi(1))$.

In order that $\phi(1)$ has finite order the eigenvalues would all have to be roots of unity. It is shown in $[17,4.7]$ by a straightforward computation that the center of $\pi$ is isomorphic to $\mathbf{Z} / n \mathbf{Z}$ if $\phi(1)$ has order $n$ and is trivial if the order of $\phi(1)$ is infinite provided that $f(1) \neq 0$. This completes the sketch. Further details can be found in $[\mathbf{1 8}, \S 2]$. 
6.12. We shall now sketch a proof of the first part of Theorem 3 of $\$ 3$. Notice that if $f(x)$ satisfies all 6 conditions, $f(1)= \pm 1$, and $F= \pm I(F= \pm I$ is guaranteed if $f(0)<0$ or if $k$ is odd or more generally if there are no roots of unity in $\mathbf{Q}(\lambda)$ other than \pm 1$)$, then

$$
\text { Out }(\pi) \simeq \mathbf{Z} / 2 \mathbf{Z} \oplus \mathbf{Z}^{s}, \text { some } s<k,
$$

and $\pi$ is centerless. That such polynomials exist is verified in [18, §2]. The aspherical manifolds $M$ that may be constructed from the polynomials fiber over the circle with fiber a $k$-torus $T^{k}$ (see $\left.[18, \S 8]\right)$. Consequently, by Theorem 2(iii), the only finite subgroups of $\mathcal{H}(M)$ are isomorphic to $\mathbf{Z} / 2 \mathbf{Z}$ and we obtain the first part of Theorem 3.

6.13. Central eXtensions. An important special case of Theorem 8 occurs when we assume that $\phi$ is trivial. Then the extension (6.1) is a central extension of $\mathbf{Z}^{k}$ by $N$. Automatically we have the compatibility condition $(\phi \circ \Phi) g=g \phi$ since $\phi$ is trivial. Furthermore, $\Gamma_{0}^{\phi}(a)=0$, and

$$
\begin{aligned}
\Gamma_{1}^{\phi}(a) & =\Gamma^{\phi}(a) \\
& =\left\{g \mid g \in \mathrm{GL}(k, \mathbf{Z}) \text { with } g_{*}(a)=a\right\}=\Gamma(a) .
\end{aligned}
$$

Also, $K_{0}=\bar{B}^{1}$ and $B^{1}\left(N ; \mathbf{Z}^{k}\right)=0$. Thus $\bar{H}^{1}\left(N ; \mathbf{Z}^{k}\right)=\operatorname{Hom}\left(N, \mathbf{Z}^{k}\right) / K_{0}$. Moreover, if $N$ is centerless, then $\operatorname{Inn}(N)=\operatorname{Inn}(\pi)=N$ and consequently $K_{0}=0$.

COROLlaRY 1. If $N /[N, N]=H_{1}(N ; \mathbf{Z})$ is a torsion group then there are the exact sequences:

$$
\begin{aligned}
& 1 \rightarrow \Gamma(a) \rightarrow \text { Aut }(\pi) \rightarrow \text { Aut }(a) \rightarrow 1, \\
& 1 \rightarrow \Gamma(a) \rightarrow \text { Out }(\pi) \rightarrow \text { Out }(a) \rightarrow 1
\end{aligned}
$$

Note simply that under the hypothesis, $\operatorname{Hom}\left(N, \mathbf{Z}^{k}\right)$ is trivial.

COROLlaRy 2. If $k=1$, and $2 a \neq 0$, then there are short exact sequences:

$$
\begin{gathered}
1 \rightarrow H^{1}(N ; \mathbf{Z}) \rightarrow \text { Aut }(\pi) \rightarrow \text { Aut }(a) \rightarrow 1, \\
1 \rightarrow \bar{H}^{1}(N ; \mathbf{Z}) \rightarrow \text { Out }(\pi) \rightarrow \text { Out }(a) \rightarrow 1 .
\end{gathered}
$$

Proof. Since GL $(1, \mathbf{Z}) \simeq \mathbf{Z} / 2 \mathbf{Z}$, and $2 a \neq 0$, it follows that $\Gamma_{1}^{\phi}(a)$ is trivial and $K_{1}=\operatorname{Hom}(N, \mathbf{Z})=H^{1}(N, \mathbf{Z})$. Also observe that if $\phi$ is not trivial when $k=1$, a similar result holds if we replace $H^{1}(N ; \mathbf{Z})$ by $\operatorname{Hom}_{\phi}(N ; \mathbf{Z})$.

We have described earlier how each automorphism of Aut $\pi$ can be written as a triple $(g, \lambda, \Phi)$ with $g, \lambda$, and $\Phi$ satisfying 6.7 . It is easily seen that the group multiplication (for $\phi$ not necessarily trivial) is given by

$$
\left(g_{2}, \lambda_{2}, \Phi_{2}\right)\left(g_{1}, \lambda_{1}, \Phi_{1}\right)=\left(g_{2} \circ g_{1}, g_{2} \lambda_{1}+\lambda_{2} \Phi_{1}, \Phi_{2} \circ \Phi_{1}\right) .
$$

If we now choose $\pi=\mathrm{Z}^{k} \times N$, where $N$ is centerless (hence $\phi$ is trivial), we see that Aut $\pi$ and Out $\pi$ have the following very nice form.

COROllary 3. For $\pi=\mathrm{Z}^{k} \times N$, we have: 


$$
\begin{aligned}
& 1 \rightarrow \operatorname{Hom}\left(N, \mathbf{Z}^{k}\right) \rightarrow \text { Aut } \pi \rightarrow \operatorname{GL}(k, \mathbf{Z}) \times \text { Aut } N \rightarrow 1, \\
& 1 \rightarrow \operatorname{Hom}\left(N, \mathbf{Z}^{k}\right) \rightarrow \text { Out } \pi \rightarrow \operatorname{GL}(k, \mathbf{Z}) \times \text { Out } N \rightarrow 1 .
\end{aligned}
$$

In fact, the groups Aut $(\pi)$ and Out $(\pi)$ are iterated semidirect products.

$$
\begin{aligned}
& \text { Aut } \pi=\left(\operatorname{Hom}\left(N, \mathbf{Z}^{k}\right) \circ \mathrm{GL}(k, \mathbf{Z})\right) \circ \text { Aut } N, \\
& \text { Out } \pi=\left(\operatorname{Hom}\left(N, \mathbf{Z}^{k}\right) \circ \mathrm{GL}(k, \mathbf{Z})\right) \circ \text { Out } N .
\end{aligned}
$$

Proof. By first choosing our representative cocycle $f$ to be trivial we may choose any $g \in \operatorname{GL}(k, \mathbf{Z}), \lambda \in \operatorname{Hom}\left(N, \mathbf{Z}^{k}\right), \Phi \in$ Aut $N$ to represent an automorphism $(g, \lambda, \Phi)$. Since $N$ is centerless $B^{0}=\Gamma_{0}=0$, and $\operatorname{Hom}\left(N, \mathbf{Z}^{k}\right)$ $=H^{1}\left(N ; \mathbf{Z}^{k}\right)=\bar{H}^{1}\left(N ; \mathbf{Z}^{k}\right)$. An examination of the group multiplication yields the semidirect product structure.

This corollary corrects an imprecise and overly enthusiastic remark [17, 4.13] concerning the structure of Aut $\pi$ and Out $\pi$. This remark was used in $[15, \S 11]$ to extend constructions made in the earlier part of [15] for manifolds of dimensions 3 and 4 to constructions in all dimensions greater than 2. It is shown there that for any $n>2$, there exist (closed) aspherical manifolds, $M_{1}^{n}$ and $M_{2}^{n}$, so that $M_{1}^{n} \times S^{1}$ is diffeomorphic to $M_{2}^{n} \times S^{1}$ but $\pi_{1}\left(M_{1}^{n}\right)$ fails to be isomorphic to $\pi_{1}\left(M_{2}^{n}\right)$. The splitting of Out $\pi \leftrightarrows$ Out $N$, is the only portion of the imprecise remark used in verifying the nonisomorphism of $\pi_{1}\left(M_{1}\right)$ and $\pi_{1}\left(M_{2}\right)$. Since we have just demonstrated this splitting, our claims, in $[15, \S 11]$, still remain valid.

6.14. $\mathbf{Z}^{k}$ NOT CHARACTERISTIC. As a final remark we mention that assumption (6.2) that $\mathbf{Z}^{k}$ be a characteristic subgroup of $\pi$ is used only in showing that every automorphism $\sigma$ of $\pi$ induces an automorphism $g$ of $\mathbf{Z}^{k}$ and $\Phi$ of $N$. If $\mathbf{Z}^{k}$ is only assumed to be normal, then replace Aut $(\pi)$ by

$$
\text { Aut }\left(\pi ; \mathbf{Z}^{k}\right)=\{\boldsymbol{\sigma} \mid \boldsymbol{\sigma} \in \text { Aut } \pi \text { and } \boldsymbol{\sigma} \text { induces }
$$

$$
\text { an automorphism of } \mathbf{Z}^{k} \text {. }
$$

Notice that $\operatorname{Inn}(\pi)=\operatorname{Inn}\left(\pi ; \mathbf{Z}^{k}\right)$, and we may define Out $\left(\pi ; \mathbf{Z}^{k}\right)$ as the quotient Aut $\left(\pi ; \mathbf{Z}^{k}\right) / \operatorname{Inn}(\pi)$. To calculate Aut $\left(\pi ; \mathbf{Z}^{k}\right)$ and Out $\left(\pi ; \mathbf{Z}^{k}\right)$ we do not need to change any of the other definitions to obtain the analogous formulae of Theorem 6. In either case it is interesting to note that $\operatorname{Hom}_{\phi}\left(N, \mathbf{Z}^{k}\right)$ are those automorphisms of $\pi$ which induce the identity on both $\mathbf{Z}^{k}$ and $N$ and $B_{\phi}^{1}\left(N, \mathbf{Z}^{k}\right)$ is identifiable with those inner automorphisms of $\pi$ arising from conjugating by elements of $\mathbf{Z}^{k}$. The cohomology group $H_{\phi}^{1}\left(N ; \mathbf{Z}^{k}\right)$ and its quotient $\bar{H}_{\phi}^{1}\left(N ; \mathbf{Z}^{k}\right)$ are particularly vulnerable to geometric realization as we shall see in $\$ 7$.

\section{Fiber preserving homeomorphisms and geometrically realizing the subgroup $K$ of Out $\pi$.}

7.1. In $\$ 6$ we obtained formulae for Out $\left(\pi ; \mathbf{Z}^{k}\right)$ in terms of automorphisms of $\mathbf{Z}^{k}$ and automorphisms of $N$. There is for any space $X$ a natural map $\Psi: \mathcal{E}(X) \rightarrow$ Out $(\pi)$. We will show that injective Seifert fiberings $\left(T^{k}, X, \phi\right)$ will furnish a class of spaces for which the composition $\Psi \circ i$ : $\mathcal{H}(X)$ $\rightarrow$ Out $\left(\pi_{1}(X)\right)$ is always onto $K \subset$ Out $\left(\pi_{1}(X)\right)$, where $K$ is the kernel of the 
epimorphism Out $\left(\pi ; \mathbf{Z}^{k}\right) \rightarrow$ Out $\left(a ; \mathbf{Z}^{k}\right)$ as defined in the preceding section. Furthermore this realization of outer automorphisms of the fundamental group by self-homeomorphisms of $X$ will have the striking property that they move each point only along its fiber.

Let $\left(T^{k}, X, \phi\right)$ be an injective Seifert fiber space in the sense of $\S 5$. Let us choose a base point $x_{0} \in X$. Passing through $x_{0}$ is an immersion of the torus $\mathrm{ev}^{x_{0}}: T^{k} \rightarrow T^{k} / N_{w_{0}}$ where $\mu\left(x_{0}\right)=\nu\left(w_{0}\right)$, and $\nu$ is the map $X^{\prime} / T^{k}=W$ $\rightarrow W / N=\mu(X)$. The "evaluation" monomorphism ev $\mathrm{v}_{*}^{x_{0}}: \pi_{1}\left(T^{k}\right) \rightarrow \pi_{1}\left(X, x_{0}\right)$ has cokernel $N$. The covering space $X^{\prime}$ of $X$, corresponding to the image of $\mathrm{ev}_{*}^{x_{0}}$, is a principal toral bundle over $X^{\prime} / T^{k}=W$. On $X^{\prime}$ we have the left-right action satisfying

$$
\left(t x^{\prime}\right) \alpha=\left(\phi\left(\alpha^{-1}\right)(t)\right)\left(x^{\prime} \alpha\right),
$$

for all $x^{\prime} \in X^{\prime}, t \in T^{k}, \alpha \in N$. Furthermore, this bundle is trivial and $X^{\prime}$ splits into $T^{k} \times W$.

Let us be explicit and choose a cross-section $W \rightarrow X^{\prime}$ to write as $T^{k} \times W$. Choose $x_{0}^{\prime}=\left(e, w_{0}\right)$, with $\nu^{\prime}\left(x_{0}^{\prime}\right)=x_{0}$, as base points and represent $x^{\prime} \in X^{\prime}$ by $(t, w) \in T^{k} \times W$. Then

$$
\begin{aligned}
\left(t^{\prime} x^{\prime}\right) \alpha & =\left(t^{\prime} t, w\right) \alpha=\left(\phi\left(\alpha^{-1}\right)\left(t^{\prime} t\right) m\left(w \alpha, \alpha^{-1}\right), w \alpha\right) \\
& =\phi\left(\alpha^{-1}\right)\left(t^{\prime}\right)\left(\phi\left(\alpha^{-1}\right)(t) m\left(w \alpha, \alpha^{-1}\right), w \alpha\right)=\phi\left(\alpha^{-1}\right)\left(t^{\prime}\right)\left(x^{\prime} \alpha\right) .
\end{aligned}
$$

Here $m$ is a function $N \times W \rightarrow T^{k}$ which, when regarded as a function from $N$ into the $\mathbf{Z}-N$ module MAPS $\left(W, T^{k}\right)$, satisfies the 1-cocycle condition. The function is obtained by considering $(e, w) \alpha=(\bar{t}, w \alpha)$ for some $\bar{t}$, which depends upon $w$ and $\alpha$. The 1-cocycle condition

$$
m(w, \alpha \beta)=m(w, \alpha) \phi(\alpha)(m(w \alpha, \beta))
$$

is obtained from the fact that $\mu^{\prime}$ is $N$-equivariant and the $\left(X^{\prime}, N\right)$ action is compatible with the toral action.

Let us represent the image of a point $x^{\prime}=(t, w)$ in $X$ under the orbit map $\nu^{\prime}$ by $\nu^{\prime}(t, w)=\langle t, w\rangle$. Thus $\langle t, w\rangle$ can be regarded as the equivalence class of pairs $(t, w)$ under the relation

$$
\langle t, w\rangle=\langle(t, w) \alpha\rangle=\left\langle\phi\left(\alpha^{-1}\right)(t) m\left(w \alpha, \alpha^{-1}\right), w \alpha\right\rangle .
$$

See $[17, \S 4]$ and $[11, \S 8]$ for further details.

We want to define a group of homeomorphisms on $X^{\prime}$ which will induce a group of homeomorphisms on $X$. In order to motivate this definition we shall consider the central case first. This is more natural and also has the desirable feature that it can be defined on $X$ directly.

7.2. The CenTral CASE. For the present, we assume that $\phi$ is trivial, and consequently, the Seifert fiber space $\left(T^{k}, X, \phi\right)$ has a toral action $\left(T^{k}, X\right)$ and the fiber map $\mu$ is simply the orbit map. Let $G\left(T^{k}, X\right)$ be the set of pairs $(g, H), g \in \mathrm{GL}(k, \mathbf{Z})=\operatorname{Aut}\left(T^{k}\right), H \in \mathscr{H}(X)$ so that $H(t x)=g(t) H(x)$, for all $x \in X, t \in T^{k}$.

Observe that this set forms a group under the multiplication $(g, H)\left(g_{1}, H_{1}\right)$ $=\left(g g_{1}, H H_{1}\right)$. Furthermore, this group can be considered a subgroup of $\mathcal{T C}(X)$ 
since $(g, H)$ and $\left(g_{1}, H\right)$, as elements of $G\left(T^{k}, X\right)$, imply $g=g_{1}$. For, $g(t) H(x)=H(t x)=g_{1}(t) H(x)$ for all $t \in T^{k}, x \in X$ implies that $g=g_{1}$ provided that the toral action $X$ is effective (which we may assume without loss of any generality).

Choose any path $\tau:(I, 0,1) \rightarrow\left(X, x_{0}, H\left(x_{0}\right)\right)$. Then $H$ induces an automorphism $H_{*}$ of $\pi_{1}\left(X, x_{0}\right)$ via the path $\tau$. The automorphism depends upon the choice $\tau$ and another choice changes $H_{*}$ by the usual inner automorphism of $\pi_{1}\left(X, x_{0}\right)$. We now wish to lift $(g, H)$ to $\left(T^{k}, X^{\prime}\right)$ so that the lifted pair $(g, h)$ satisfies

$$
h\left(x^{\prime} \alpha\right)=h\left(x^{\prime}\right) H_{*}(\alpha) \text { and } h\left(t x^{\prime}\right)=g(t) h\left(x^{\prime}\right) .
$$

Recall that $\operatorname{ev}_{*}^{x}: \pi_{1}\left(T^{k}, 1\right) \rightarrow \pi_{1}(X, x)$ does not depend upon $x$. That is, if $\sigma \in \pi_{1}\left(T^{k}, 1\right)$ then $\mathrm{ev}_{*}^{x_{0}}(\sigma)=\gamma^{-1} \mathrm{ev}_{*}^{x}(\sigma) \gamma$, for any choice $\gamma:(I, 0,1) \rightarrow(X$, $\left.x_{0}, x\right)$. Therefore, we see that $H_{*}(\sigma)=g(\sigma)$. If we consider the mapping $H \circ \nu^{\prime}:\left(X^{\prime}\right) \rightarrow X$, the image $\left(\pi_{1}\left(X^{\prime}, x_{0}^{\prime}\right)\right)$ in $\pi_{1}\left(X, H\left(x_{0}\right)\right)$ is precisely $\operatorname{im}\left(\operatorname{ev}_{*}^{x} g\left(\pi_{1}\left(T^{k}, 1\right)\right)\right)$ and there is a mapping $h:\left(X^{\prime}, x_{0}^{\prime}\right) \rightarrow\left(X^{\prime}, y^{\prime}\right)$ covering $H$. Now $\nu^{\prime}\left(h\left(t x^{\prime}\right)\right)=H\left(\nu^{\prime}\left(t x^{\prime}\right)\right)=H(t x)=g(t) H(x)$. Since $\nu^{\prime}\left(g(t) h\left(x^{\prime}\right)\right)$ $=g(t) \nu^{\prime}(h(x))=g(t) H(x)$, we have that $h\left(t x^{\prime}\right)$ and $g(t) h\left(x^{\prime}\right)$ both cover $H(t x)$. Uniqueness of lifting with specified base points and the connectedness of $T^{k}$ yields the formula

$$
g(t) h\left(x^{\prime}\right)=h\left(t x^{\prime}\right) .
$$

The automorphism $H_{*}$, which reduces to $g$ on $\operatorname{im}\left(\mathrm{ev}_{*}^{x_{0}}\right)$, induces an

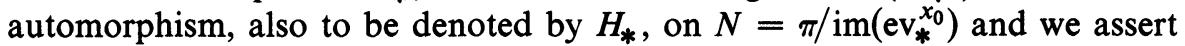
$h\left(x^{\prime} \alpha\right)=h\left(x^{\prime}\right) H_{*}(\alpha)$, for all $\alpha \in N$. Since $h\left(x^{\prime}\right) \rightarrow h\left(x^{\prime} \alpha\right)$ is a covering transformation it is only necessary to check this at $x^{\prime}=x_{0}^{\prime}$, and there it is straightforward. We may also inquire what other possible lifts are there? The different lifts are in 1-1 correspondence with the elements of $N$, or equivalently with $\left(\nu^{\prime}\right)^{-1}\left(H\left(x_{0}\right)\right)$. In fact, if we let $h^{\tau}$ denote the lift of $H$ corresponding to the path class of $\tau$, and $H_{*}^{\tau}$ the induced automorphisms on $\pi$ and $N$, then

$$
H_{*}^{\tau}(\alpha)=\gamma H_{*}^{\tau}(\alpha) \gamma^{-1},
$$

where $\gamma$ is the class of the loop $\left(\tau_{1}\right)^{-1} \tau$. Thus

$$
h^{\tau_{1}}\left(x^{\prime} \cdot \alpha\right)=h^{\tau_{1}}\left(x^{\prime}\right) H_{*}^{\tau^{\prime}}(\alpha)=h^{\tau_{1}}\left(x^{\prime}\right)\left(\gamma H_{*}^{\tau}(\alpha) \gamma^{-1}\right),
$$

and $H_{*}(\alpha)$ is determined up to inner automorphisms of $\pi / \operatorname{im}\left(\mathrm{ev}_{*}^{x_{0}}\right)=N$.

Now on $X^{\prime} / T^{k}=W$, there is induced a homeomorphism $\hat{h}: W \rightarrow W$ with the property $\hat{h}(w \alpha)=\hat{h}(w) H_{*}(\alpha)$, for all $w \in W$ and $\alpha \in N$. This says that the action of $N$ induced by the automorphism $H_{*}$ is equivalent (equivariantly homeomorphic) to the original action of $N$ on $W$.

7.3. THE GENERAL CASE: THE GROUP OF FIBER PRESERVING HOMEOMORPHISMS. With the foregoing in mind we now wish to examine the general case where $\phi$ is not necessarily trivial. Instead of beginning with $X$, we begin with $\left(X^{\prime}, x_{0}^{\prime}\right)$. We consider now pairs $(g, h), g \in \mathrm{GL}(k, \mathbf{Z})=$ Aut $\left(T^{k}\right), h: X^{\prime} \rightarrow X^{\prime}=$ $T^{k} \times W$ a homeomorphism satisfying:

1. $h\left(t x^{\prime}\right)=g(t) h\left(x^{\prime}\right)$, 
2. $h\left(x^{\prime} \alpha\right)=h\left(x^{\prime}\right) \cdot H_{*}(\alpha)$ for some automorphism $H_{*}$ of $N$.

We need to explain the automorphism $H_{*}$ of $N$. Since $h\left(t x^{\prime}\right)=g(t) h\left(x^{\prime}\right)$, there is induced on $W=X^{\prime} / T^{k}$ a homeomorphism $\hat{h}$, defined by $\hat{h}\left(\mu^{\prime}\left(x^{\prime}\right)\right)$ $=\mu^{\prime}\left(h\left(x^{\prime}\right)\right)$. Condition 2 asserts that $h$ induces a homeomorphism $H$ on $X$. The two conditions together with $\left(t x^{\prime}\right) \alpha=\phi\left(\alpha^{-1}\right)(t)\left(x^{\prime} \cdot \alpha\right)$ imply

3. $g\left(\phi\left(\alpha^{-1}\right)(t)\right)=\phi\left(H_{*}\left(\alpha^{-1}\right)\right) g(t)$.

The sets of pairs $(g, h)$ satisfying conditions 1 and 2 form a group under the law of composition $(g, h)\left(g_{1}, h_{1}\right)=\left(g \circ g_{1}, h \circ h_{1}\right)$. To check the group law condition 3 is useful. Once again, given pairs $(g, h)$ and $\left(g_{1}, h\right), g=g_{1}$ and this group can be regarded as a subgroup of $\mathcal{H}\left(X^{\prime}\right)$. We denote this group by $G\left(T^{k}, X^{\prime}, \phi\right)$ and its image in $\mathcal{H}(X)$ by $G\left(T^{k}, X, \phi\right)$. We call the latter the group of fiber preserving homeomorphisms of the Seifert fibering $\left(T^{k}, X, \phi\right)$.

Let us also use $\nu^{\prime}$ to denote the projection $G\left(T^{k}, X^{\prime} \phi\right) \rightarrow G\left(T^{k}, X, \phi\right)$ under the homomorphism $(g, h) \rightarrow(g, H)$. We wish to study $\left(\nu^{\prime}(g, h)\right)_{*}: \pi_{1}\left(X, x_{0}\right)$ $\rightarrow \pi_{1}\left(X, H\left(x_{0}\right)\right)$. Choose a path $\tau^{\prime}:(I, 0,1) \rightarrow\left(X^{\prime}, x_{0}^{\prime}, h\left(x_{0}^{\prime}\right)\right)$ and let $\tau=\nu^{\prime}\left(\tau^{\prime}\right)$. The homeomorphism $H$ induces an automorphism $\bar{H}_{*}$ of $\pi_{1}\left(X, x_{0}\right)$ via the path $\tau$. On the image $\nu_{*}^{\prime}\left(\pi_{1}\left(T^{k}, 1\right) \approx \mathbf{Z}^{k}, \bar{H}_{*}\right.$ is invariant and is given by $g$ from (1). Therefore on $N$ there is induced an automorphism which we shall also denote by $\bar{H}_{*}$. If we choose a different path $\tau_{1}^{\prime}$ but with the same endpoints, then $\bar{H}_{*}$ via the new path $\tau_{1}$ is altered by an inner automorphism obtained by conjugating by $\tau_{1}^{-1} \tau$. Thus $\bar{H}_{*}$ restricted to $\mathbf{Z}^{k}$ is unchanged, and $\bar{H}_{*}$ induced on $N$ is also unchanged. In any case, the unique lifting of $H$ to $X^{\prime}$ so that the image of $x_{0}^{\prime}$ is $h\left(x_{0}^{\prime}\right)$ is, of course, $h$. Now $h\left(x^{\prime} \cdot \alpha\right)=h\left(x^{\prime}\right) \cdot H_{*}(\alpha)$ means that $h\left(x^{\prime}\right) \rightarrow h\left(x^{\prime} \cdot \alpha\right)$ is a covering transformation and so is determined by what it does on the base point $x_{0}^{\prime}$. Let $\sigma:(I, 0,1) \rightarrow\left(X, x_{0}, x_{0}\right)$ represent a class $\alpha \in \pi_{1}\left(X, x_{0}\right) / \nu_{*}^{\prime}\left(\pi_{1}\left(X^{\prime}, x_{0}{ }^{\prime}\right)\right)=N$. To find $h\left(x_{0}^{\prime} \alpha\right)$ we just need to consider the lift of the path $H(\sigma)$ at $H\left(x_{0}\right)$ to the endpoint of $\tau^{\prime}$. This is the lift of the path $H(\sigma) \cdot \tau$. To find $h\left(x_{0}^{\prime}\right) \cdot \bar{H}_{*}(\alpha)$ this is the lift of the path $\tau\left(\tau^{-1} H(\sigma) \tau\right)$ which of course has the same endpoint. Thus $h\left(x_{0}^{\prime}\right) \cdot \bar{H}_{*}(\alpha)=h\left(x_{0}^{\prime}\right) H_{*}(\alpha)$ $=h\left(x_{0}^{\prime} \alpha\right)$. That is $H_{*}$ on $N$ is precisely $\bar{H}_{*}$. Thus the pair $(g, h) \in G\left(T^{k}, X^{\prime}, \phi\right)$ determines an automorphism $\bar{H}_{*}$ of $\pi$ (modulo conjugation by elements in $\mathbf{Z}^{k}$ ) and restricts to $g$ on $\mathbf{Z}^{k}$ and determines a unique $H_{*} \in$ Aut $(N)$. We shall now drop the bar over the $H$. On the other hand, given $H \in G\left(T^{k}, X, \phi\right), H_{*}$ induces automorphisms on $\mathbf{Z}^{k}$ and $N$ which are uniquely determined up to conjugation by elements of $\pi$. An explicit formula will be obtained in the lemma of 7.6. In any case $H_{*}$ determines a unique element of Out $\pi$.

Our Seifert fibering $\mu$ with the extension

$$
1 \rightarrow \pi_{1}\left(X^{\prime}, x_{0}^{\prime}\right) \rightarrow \pi_{1}\left(X, x_{0}\right) \rightarrow N \rightarrow 1
$$

is given by its Bieberbach class $a \in H_{\phi}^{2}\left(N ; \mathbf{Z}^{k}\right)$. It will be convenient to have an explicit formula stating that

7.4. LEMMA. $H^{*}(a)=g_{*}(a)$.

That is, $H_{*}$ in $\operatorname{Aut}(N)$ determines uniquely an element in $\operatorname{Out}(a)$ $\subseteq \operatorname{Out}(N)$. We simplify notation by denoting the isomorphism on cohomology by $H^{*}$ instead of $\left(H_{*}\right)^{*}$. In order to show that $H^{*}(a)=g_{*}(a)$ we recall $[17, \S \S 3,4]$ and $[11, \S 8]$ that

$$
\delta: H_{\phi}^{1}\left(N ; \operatorname{MAPS}\left(W, T^{k}\right)\right) \cong H_{\phi}^{2}\left(N ; \mathbf{Z}^{k}\right)
$$


and corresponding to $a$ there is a map $m: W \times N \rightarrow T^{k}$ satisfying

$$
m(w, \alpha) \phi(\alpha) m(w \alpha, \beta)=m(w, \alpha \beta)
$$

so that $\left(T^{k}, X^{\prime}, N\right)$ can be represented up to $T^{k}-N$ equivalence by

$$
(t, w) \alpha=\left(\phi\left(\alpha^{-1}(t) m\left(w \alpha, \alpha^{-1}\right), w \alpha\right)\right)
$$

To recover $(g, h)$ explicitly on $X^{\prime}$ we note that there is a function $\lambda: W$ $\rightarrow T^{k}$ with $h(e, w)=(\lambda(w), \hat{h}(w))$ and thus

$$
h(t, w)=g(t) h(e, w)=(g(t) \lambda(w), \hat{h}(w)) .
$$

We know $h\left(x^{\prime} \cdot \alpha^{-1}\right)=h\left(x^{\prime}\right) H_{*}\left(\alpha^{-1}\right)$. Put $x^{\prime}=(t, w \alpha)$. We have

$$
\begin{aligned}
h\left((t, w \alpha) \alpha^{-1}\right) & =h(\phi(\alpha)(t) m(w, \alpha), w) \\
& =(g(\phi(\alpha)(t)) g(m(w, \alpha)) \lambda(w), \hat{h}(w)) .
\end{aligned}
$$

This is equal to

$$
\begin{aligned}
& (h(t, w \alpha)) H_{*}\left(\alpha^{-1}\right)=(g(t) \lambda(w \alpha), \hat{h}(w \alpha)) H_{*}\left(\alpha^{-1}\right) \\
& \quad=\left(\phi\left(H_{*}(\alpha)\right)(g(t) \lambda(w \alpha)) m\left(\hat{h}(w \alpha) H_{*}\left(\alpha^{-1}\right), H_{*}(\alpha)\right), \hat{h}(w \alpha) H_{*}\left(\alpha^{-1}\right)\right) \\
& \quad=\left(\phi\left(H_{*}(\alpha)\right)(g(t)) \phi\left(H_{*}(\alpha)\right)(\lambda(w \alpha)) m\left(\hat{h}(w), H_{*}(\alpha)\right), \hat{h}(w)\right) .
\end{aligned}
$$

Since $g(\phi(\alpha)(t))=\phi\left(H_{*}(\alpha)\right) g(t)$, we have

$$
g(m(w, \alpha))=\phi\left(H_{*}(\alpha)\right)(\lambda(w \alpha)) m\left(\hat{h}(w), H_{*}(\alpha)\right) \lambda(w)^{-1} .
$$

But the operators on $H^{*}(a)$ are given by $\phi \circ H_{*}$. Thus this condition says precisely that $g_{*}(m)=\Phi^{*}(m)$. Under

$$
\delta: H_{\phi}^{1}\left(N ; \operatorname{MAPS}\left(w, T^{k}\right)\right) \stackrel{\simeq}{\longrightarrow} H_{\phi}^{2}\left(N ; \mathbf{Z}^{k}\right),
$$

$g(m(w, \alpha))$ corresponds to $g_{*}(a)$ while $m\left(\hat{h}(w), H_{*}(\alpha)\right)$ corresponds to $H^{*}(a)$. Consequently, $g_{*}(a)=H^{*}(a)$, which completes the proof of the lemma.

Thus, if we choose to represent the injective Seifert fiber space $\left(T^{k}, X, \phi\right)$ explicitly by choosing a cross-section $\chi: X^{\prime} / T^{k}=W \rightarrow X^{\prime}$ and a 1-cocycle $m \in Z^{1}\left(N\right.$; MAPS $\left.\left(W, T^{k}\right)\right)$, we may represent each element of $G\left(T^{k}, X^{\prime}, \phi\right)$ explicitly by a triple $(g, \lambda, \hat{h})$ so that $(7.5)$ is satisfied. Note that composition is given by

$$
\left(g_{1}, \lambda_{1}, \hat{h}_{1}\right) \circ\left(g_{2}, \lambda_{2}, \hat{h}_{2}\right)=\left(g_{1} \circ g_{2},\left(g_{1} \lambda_{2}\right)\left(\lambda_{1} \circ \hat{h}_{2}\right), \hat{h}_{1} \circ \hat{h}_{2}\right)
$$

which also gives an explicit representation of the homomorphism

$$
\tilde{\Psi}=\Psi \circ i \circ \nu^{\prime}: G\left(T^{k}, X^{\prime}, \phi\right) \rightarrow \text { Out } \pi_{1}\left(X, x_{0}\right) .
$$

Recall that $K$ is the subgroup of Aut $\pi$ which induces the identity on $N$ reduced by those arising from conjugation in $\pi$.

7.6. TheOREM 9. $G\left(T^{k}, X^{\prime}, \phi\right)$ contains a subgroup $G_{1}\left(T^{k}, X^{\prime}, \phi\right)$ which is mapped by $\tilde{\Psi}$ homomorphically onto $K \subset$ Out $\pi$. 
Of course, this says that every outer automorphism of $\pi_{1}(X)$, which is in $K$, can be represented by a fiber preserving homeomorphism.

Proof. We define $G_{1}\left(T^{k}, X^{\prime}, \phi\right)$ as the subgroup of triples $(g, \lambda, \hat{h})$ so that $\hat{h}=\mathrm{id}: W \rightarrow W$. In $G_{1}$, we take the subgroup

$$
G_{2}=\{(g, \lambda, \hat{h})=(I, \lambda, \mathrm{id})\} .
$$

Note $G_{2}$ is the set of functions $\lambda: W \rightarrow T^{k}$ so that $\phi(\alpha) \lambda(w \alpha)=\lambda(w)$ by (7.5), for all $\alpha \in N, w \in W$. Such a function is an element of

$$
H_{\phi}^{0}\left(N ; \operatorname{MAPS}\left(W, T^{k}\right)\right) .
$$

The action on $X^{\prime}$ is given by

$$
(I, \lambda, \mathrm{id})(t, w)=(t \lambda(w), w)=h(t, w)
$$

and on $X$ by

$$
\nu^{\prime}(I, \lambda, \mathrm{id})\langle t, w\rangle=\langle t \lambda(w), w\rangle .
$$

We shall check that every $\lambda \in H_{\phi}^{0}\left(N ; \operatorname{MAPS}\left(W, T^{k}\right)\right)$ satisfies conditions 1 and 2 and is therefore an element of $G\left(T^{k}, X^{\prime}, \phi\right)$.

1. $h\left(t^{\prime}(t, w)\right)=h\left(t^{\prime} t, w\right)=t^{\prime}(t \lambda(w), w)$,

2. $h((t, w) \alpha)=h\left(\phi\left(\alpha^{-1}\right)(t) m\left(w \alpha, \alpha^{-1}\right), w \alpha\right)$

$$
\begin{aligned}
& =\left(\phi\left(\alpha^{-1}\right)(t) m\left(w \alpha, \alpha^{-1}\right) \lambda(w), w \alpha\right) \\
& =\left(\phi\left(\alpha^{-1}\right)((t) \lambda(w)) m\left(w \alpha, \alpha^{-1}\right), w \alpha\right)=(h(t, w)) \alpha .
\end{aligned}
$$

Thus,

$$
\nu^{\prime}(h)\langle t, w\rangle=H\langle t, w\rangle=\langle t \lambda(w), w\rangle .
$$

As a pair $(I, H)=\nu^{\prime}(I, \lambda$, id $)$ induces an automorphism $H_{*}$ of $\pi$ which is the identity on $\mathbf{Z}^{k}$ and the identity on $N . H_{*}$ as an automorphism belongs to $\operatorname{Hom}_{\phi}\left(N, \mathbf{Z}^{k}\right)$. However in defining $H_{*}$, a choice of path $\tau^{\prime}:(I, 0,1) \rightarrow\left(X^{\prime}, x_{0}^{\prime}\right.$, $\left.h\left(x_{0}^{\prime}\right)\right)$ has to be made and we use $\nu^{\prime}\left(\tau^{\prime}\right)=\tau$. This can alter our choice of $H_{*}$ by conjugation by an element of $\mathbf{Z}^{k}$. But this conjugation corresponds to an element of $B_{\phi}^{1}\left(N ; \mathbf{Z}^{k}\right)$ according to the proof of Theorem 8 . Thus each choice of $\lambda \in H_{\phi}^{0}\left(N\right.$; MAPS $\left.\left(W, T^{k}\right)\right)$ gives rise to a uniquely defined homeomorphism $H \in \nu^{\prime}\left(G_{2}\right) \subset G\left(T^{k}, X, \phi\right)$ so that $H_{*}$ is uniquely determined as an element of $H_{\phi}^{1}\left(N ; \mathbf{Z}^{k}\right)$. It remains to check that $\delta \lambda=H_{*}$.

The coboundary $\delta: H_{\phi}^{0}\left(N ; \operatorname{MAPS}\left(W, T^{k}\right)\right) \rightarrow H_{\phi}^{1}\left(N ; \mathbf{Z}^{k}\right) \rightarrow 0$ is induced from the cohomology exact sequence which itself is induced by the exact coefficient sequence

$$
0 \rightarrow \operatorname{MAPS}\left(W, \mathbf{Z}^{k}\right) \rightarrow \operatorname{MAPS}\left(W, \mathbf{R}^{k}\right) \rightarrow \operatorname{MAPS}\left(W, T^{k}\right) \rightarrow 0 .
$$

The module structure on MAPS $\left(W, T^{k}\right)$ is given by

$$
\left(\alpha_{\sharp} f\right)(w)=\phi(\alpha) f(w \alpha) .
$$

Since $H_{\phi}^{i}\left(N\right.$; MAPS $\left.\left(W, \mathbf{R}^{k}\right)\right)=0$, for $i>0[17,12.1], \delta H^{0}$ is surjective and 
$\delta H^{i}$ is an isomorphism, $i>0$. Now we observe that by taking the coboundary of (7.5) we get (6.7), which verifies our assertion $\delta \lambda=H_{*}$. However, for the skeptical, it won't hurt to point out that $\delta \lambda_{1}=\delta \lambda_{2}$ if and only if $\lambda_{1} \lambda_{2}^{-1}$ $=\exp (2 \pi i \Lambda)$, where $\Lambda \in H_{\phi}^{0}\left(N ; \operatorname{MAPS}\left(W, \mathbf{R}^{k}\right)\right)$. Note

$$
\Lambda_{s}=s \Lambda \in H_{\phi}^{0}\left(N ; \operatorname{MAPS}\left(W, \mathbf{R}^{k}\right)\right), \quad 0 \leqslant s \leqslant 1 .
$$

Thus, $\exp \left(2 \pi i \Lambda_{s}\right) \lambda_{2}$ is a homotopy between $\lambda_{1}$ and $\lambda_{2}$ so that at each stage $\exp \left(2 \pi i \Lambda_{s}\right) \Lambda_{2} \in H_{\phi}^{0}\left(N ; \operatorname{MAPS}\left(W, T^{k}\right)\right)$. Thus $\left(I, \lambda_{1}, h_{1}\right)$ and $\left(I, \lambda_{2}, h_{2}\right)$ are equivariantly isotopic and induce an isotopy between $\nu^{\prime}\left(I, \lambda_{1}, h_{1}\right)=\left(I, H_{1}\right)$ and $\nu^{\prime}\left(I, \lambda_{2}, h_{2}\right)=\left(I, H_{2}\right)$. The "base" $W / N$ is left fixed and so the isotopy moves just in each fiber.

We still need to explain $H_{\phi}^{1}\left(N ; \mathbf{Z}^{k}\right) \rightarrow \bar{H}_{\phi}^{1}\left(N ; \mathbf{Z}^{k}\right)$. This arises because $\nu^{\prime}: G\left(T^{k}, X^{\prime}, \phi\right) \rightarrow G\left(T^{k}, X, \phi\right)$ has kernel precisely the covering transformations $N$. Recall that we choose a base point $x_{0}^{\prime}$ over $x_{0}$. If $h \in G\left(T^{k}, X^{\prime}, \phi\right)$, then define

$$
(h \times \alpha)\left(x^{\prime}\right)=h\left(x^{\prime}\right) H_{*}(\alpha),
$$

where $h\left(x^{\prime} \alpha\right)=h\left(x^{\prime}\right) H_{*}(\alpha)$, for all $\alpha \in N . h \times \alpha$ also has the same image as $h$ in $G\left(T^{k}, X, \phi\right)$. That is, $\nu^{\prime}(h)=\nu^{\prime}(h \times \alpha)$. To make precise which elements, as pairs, correspond to $h \times \alpha$ we have

Lemma. If $h \in G\left(T^{k}, X^{\prime}, \phi\right)$ then $h \times \alpha \in G\left(T^{k}, X^{\prime}, \phi\right)$ and

1. $(h \times \alpha)\left(t x^{\prime}\right)=\left(\phi\left(H_{*}\left(\alpha^{-1}\right)\right)(g(t))(h \times \alpha)\left(x^{\prime}\right)\right)$,

2. $(h \times \alpha)\left(x^{\prime} \beta\right)=(h \times \alpha)\left(x^{\prime}\right) H_{*}\left(\alpha^{-1} \beta \alpha\right)$. Furthermore,

$$
(h \times \alpha) \times \beta=h \times \alpha \beta .
$$

From these formulae, we see that $\nu^{\prime}(h \times \alpha)=\nu^{\prime}(h)$. The induced homomorphism $(h \times \alpha)_{*}$ is a conjugation by $\alpha$ composed with $H_{*}$. This corresponds to taking either different base points in $X^{\prime}$ (all projecting to $x_{0}$ ) or equivalently taking the different possible lifts for $H$.

Proof. Since $h\left(x^{\prime} \alpha\right)=h\left(x^{\prime}\right) H_{*}(\alpha)$, for all $\alpha \in N$, then

$$
\nu^{\prime}\left(h\left(x^{\prime} \alpha\right)\right)=\nu^{\prime}(h \times \alpha)\left(x^{\prime}\right)=\nu^{\prime}\left(h\left(x^{\prime}\right)\right) .
$$

Thus, $\nu^{\prime}(h \times \alpha)=\nu^{\prime}(h)=H$, and $h \times \alpha$ is a lift of $H$. Now,

$$
\begin{aligned}
(h \times \alpha)\left(t x^{\prime}\right) & =\left(h\left(t x^{\prime}\right)\right) H_{*}(\alpha)=\left(g(t) h\left(x^{\prime}\right)\right) H_{*}(\alpha) \\
& =\left(\phi\left(H_{*}\left(\alpha^{-1}\right)\right)\right)(g(t))\left(h\left(x^{\prime}\right)\right) \cdot\left(H_{*}(\alpha)\right) \\
& =\phi\left(H_{*}\left(\alpha^{-1}\right)\right)(g(t))(h \times \alpha)\left(x^{\prime}\right),
\end{aligned}
$$

and 1 holds. For 2,

$$
\begin{aligned}
(h \times \alpha)\left(x^{\prime} \beta\right) & =h\left(x^{\prime} \beta\right) H_{*}(\alpha)=h\left(x^{\prime} \beta \alpha\right)=h\left(x^{\prime} \alpha \alpha^{-1} \beta \alpha\right)=h\left(x^{\prime} \alpha\right) H_{*}\left(\alpha^{-1} \beta \alpha\right) \\
& =(h \times \alpha)\left(x^{\prime}\right) H_{*}\left(\alpha^{-1} \beta \alpha\right) .
\end{aligned}
$$

For $h \in G_{2}$ we observe that $(h(t, w)) \alpha=h((t, w) \alpha)$. Thus $G_{2} \times N$ is con- 
tained in the homeomorphisms of $X^{\prime}$ with $(h \times \alpha)(t, w)=(t \lambda(w), w) \cdot \alpha$. But $G_{2} \times N$ is not necessarily in $G_{1}\left(T^{k}, X^{\prime}, \phi\right)$. However, we see that the subgroup of $G_{2} \times N$ with $\alpha \in \mathscr{Z}(N)$ and $\phi(\alpha)$ trivial is a subgroup of $G_{1}\left(T^{k}, X, \phi\right)$ and maps onto $H_{\phi}^{1}\left(N ; \mathbf{Z}^{k}\right)$. Notice that $\nu^{\prime}(h)=\nu^{\prime}(h \times \alpha)$ and $\left(\nu^{\prime}(h)\right)_{*}$ differs from $\left(\nu^{\prime}(h \times \alpha)\right)_{*}$, as an automorphism of $\pi$, by an element of $\bar{B}_{\phi}^{l}\left(N ; \mathbf{Z}^{k}\right)$ modulo elements of $B^{1}\left(N ; \mathbf{Z}^{k}\right)$.

COROLlaRY 1. The group $G_{2}\left(T^{k}, X^{\prime}, \phi\right)$ is mapped homomorphically onto $\bar{H}_{\phi}^{1}\left(N ; \mathbf{Z}^{k}\right)$ by $\bar{\Psi}$. If $\bar{\Psi}\left(h_{1}\right)=\bar{\Psi}\left(h_{2}\right)$, then $\left(I, H_{1}\right)$ is isotopic to $\left(I, H_{2}\right)$ with the points moving only along the fibers.

We must now investigate the image of elements in $G_{1}\left(T^{k}, X^{\prime}, \phi\right)$ not in $G_{2}\left(T^{k}, X^{\prime}, \phi\right)$. Consider $g \in \Gamma_{1}^{\phi}(a)$. Then $g_{*}(a)=a$. If we represent $\tilde{m}$ $\in Z_{\phi}^{1}\left(N ; \operatorname{MAPS}\left(W, T^{k}\right)\right)$, where $\tilde{m}(\alpha)(w)=m(w, \alpha)$, a 1-cocycle, so that $\delta(\tilde{m})=a$, then $g_{*}(a)=a$. In terms of $\tilde{m}$ this is

$$
g \tilde{m}(\alpha)(\tilde{m}(\alpha))^{-1}=\left(\alpha_{\sharp} \lambda\right)(\lambda)^{-1},
$$

for some function $\lambda: W \rightarrow T^{k}$. In terms of its values on $W$ this says

$$
g(m(w, \alpha))(m(w, \alpha))^{-1}=\phi(\alpha)(\lambda(w \alpha))(\lambda(w))^{-1} .
$$

Let us define $h_{g}(t, w)=(g(t) \lambda(w), w)$. We check conditions 1 and 2:

1.

$$
\begin{aligned}
h_{g}\left(t^{\prime}(t, w)\right) & =h_{g}\left(t^{\prime} t, w\right)=\left(g\left(t^{\prime} t\right) \lambda(w), w\right) \\
& =g\left(t^{\prime}\right)(g(t) \lambda(w), w)=g\left(t^{\prime}\right) h_{g}(t, w), \\
h_{g}((t, w) \alpha) & =h_{g}\left(\phi\left(\alpha^{-1}\right)(t) m\left(w \alpha, \alpha^{-1}\right), w \cdot \alpha\right) \\
& =\left(g\left(\phi\left(\alpha^{-1}\right)(t)\right) g\left(m\left(w \alpha, \alpha^{-1}\right)\right) \lambda(w \alpha), w \cdot \alpha\right) .
\end{aligned}
$$

But, $g \phi=\phi g$ if $g_{*}(a)=a$, and

$$
\begin{aligned}
& \left(g \tilde{m}\left(\alpha^{-1}\right)(w \alpha)\right)\left(\tilde{m}\left(\alpha^{-1}\right)(w \alpha)\right)^{-1} \\
& \quad=g m\left(w \alpha, \alpha^{-1}\right)\left(m\left(w \alpha, \alpha^{-1}\right)\right)^{-1}=\phi\left(\alpha^{-1}\right)(\lambda(w))(\lambda(w \alpha))^{-1} .
\end{aligned}
$$

Thus

$$
\begin{aligned}
h_{g}((t, w) \alpha) & =\left(\phi\left(\alpha^{-1}\right)(g(t) \lambda(w)) m\left(w \alpha, \alpha^{-1}\right), w \cdot \alpha\right) \\
& =(g(t) \lambda(w), w) \cdot \alpha=\left(h_{g}(t, w)\right) \cdot \alpha .
\end{aligned}
$$

Therefore, we may define $\hat{h}=$ id and

$$
H_{g}\langle t, w\rangle=\left\langle h_{g}(t, w)\right\rangle=\langle g(t) \lambda(w), w\rangle .
$$

Notice that $\left(H_{g}\right)_{*}$ on $\mathbf{Z}^{k}$ is $g$, and on $N$ is identity. Our formula for $g_{*}(a)=a$ when substituted in (7.5) with $H_{*}=$ identity, $\hat{h}=$ identity, represents an element of $K_{1}^{\phi}(a)$, by $(g, \lambda$, id $)$ which maps onto $g$ by $K_{1}^{\phi}(a) \rightarrow \Gamma_{1}^{\phi}(a)$. Now we may alter $\left(g, \lambda\right.$, id) by any $\lambda_{1} \in H_{\phi}^{0}\left(N\right.$; MAPS $\left(W, T^{k}\right)$ ) (recall $\alpha_{\sharp}\left(\lambda_{1}\right) \lambda_{1}^{-1}$ $=1)$ to get $\left(g, \lambda \lambda_{1}\right.$, id). Thus every element of $K_{1}^{\phi}(a)$ is represented. Of course 
$h_{\left(g, \lambda \lambda_{1}, \text { id }\right)}$ and $h_{\left(g, \lambda \lambda_{2}, \text { id }\right)}$ are related by an equivariant isotopy keeping $W$ fixed, provided that $\delta \lambda_{1}$ is equal to $\delta \lambda_{2}$. This projects onto an isotopy of $H_{\left(g, \lambda \lambda_{1}, \text { id }\right)}$ to $H_{\left(g, \lambda \lambda_{2}, \text { id }\right)}$ which just moves each point along its fiber. We may also show, as above, that the image of $\tilde{\Psi}: G_{1}\left(T^{k}, X, \phi\right) \rightarrow K$ is obtained by dividing out $K_{0}$.

7.7. [It should be pointed out that for our definitions of $h$ and $H$ we have fixed a 1-cocycle $m(w, \alpha)$ representative so that $\delta(\tilde{m})=a \in H_{\phi}^{2}\left(N ; \mathbf{Z}^{k}\right)$, as well as having fixed a particular cross-section $\chi: W \rightarrow X^{\prime}$, that is a representation of $X^{\prime}$ as $T^{k} \times W$. We may inquire as to how our constructions depend upon these choices. We describe the $N$ action given by $m_{1}$ and $\chi_{1}$ in terms of an $m_{2}$ and $\chi_{2}$. If $\chi_{2}(w)=\lambda(w) \chi_{1}(w)$ then

$$
m_{2}\left(w \alpha, \alpha^{-1}\right) \lambda(w \alpha)=\phi\left(\alpha^{-1}\right)(\lambda(w)) m_{1}\left(w \alpha, \alpha^{-1}\right),
$$

or equivalently,

$$
m_{2}(w, \alpha) \lambda(w)=\phi(\alpha)(\lambda(w \alpha)) m_{1}(w, \alpha) .
$$

That is, $m_{2}$ is cohomologous to $m_{1}$. Similarly, given $m_{1}$ and $m_{2}$, cohomologous 1-cocycles, and $N_{1}$ and $N_{2}$, the induced $N$ actions on $X^{\prime}$ given in terms of a cross-section $\chi_{1}$, then there is a $T_{k} \circ N$ equivariant homeomorphism $(t, w)$ $\rightarrow(t \lambda(w), w)$. Thus the second action given by $m_{2}$ can also be thought of describing the first action given by $m_{1}$ but determined by the cross-section $\chi_{2}(w)=(\lambda(w), w)$. Compare $[17,4.3]$ and $[11, \S 8]$. Thus we have lost no generality in picking a fixed cocycle representative for

$$
\delta^{-1}(a) \in H_{\phi}^{1}\left(N ; \operatorname{MAPS}\left(W, T^{k}\right)\right)
$$

and a fixed cross-section of $X^{\prime}$.] This completes the proof of Theorem 9.

7.8. IsoTору. To summarize the isotopy statements that we made during the course of the proof let $H_{1}$ and $H_{2}$ be two elements of $G_{2}\left(T^{k}, X, \phi\right)$.

Corollary 2. $\left(H_{1}\right)_{*}=\left(H_{2}\right)_{*}$ in Out $\pi$, if and only if, $H_{1}$ and $H_{2}$ are homotopic if and only if, they are isotopic moving just along fibers.

Corollary 3. If, in addition, $\left(T^{k}, X, \phi\right)$ is a $K(\pi, 1)$-space then every homotopy equivalence $f: X \rightarrow X$ so that $f_{*}: \pi_{1}(X) \rightarrow \pi_{1}(X)$ represents an element of $K$ in Out $\pi$, implies $f$ is homotopic to an automorphism of the Seifert fibering so that the induced homeomorphism on $W / N$ is trivial.

7.9. ExAMPLE. This is the geometric analogue of 6.11 . Let $Y$ be a fiber bundle over the circle with fiber a $k$-torus. $Y$ can be regarded as the "mapping torus" of a homeomorphism $k: T^{k} \rightarrow T^{k} . k$ is homotopic to an element $\gamma \in \mathrm{GL}(k, \mathbf{Z})$ and so $Y$ is fiber homotopically equivalent to $\left(T^{k}, X, \gamma\right)$. If $k$ is isotopic to $\gamma$ then $Y$ is bundle equivalent to the fiber $\left(T^{k}, X, \gamma\right)$. Let $f(t)$ $=\operatorname{det}(I t-\gamma)$ be the characteristic polynomial of $\gamma$.

Proposition. If

1. $f(1) \neq 0$, and

2. $f(t) \not \equiv t^{k} f(1 / t) / f(0)$,

then every homotopy equivalence $f: X \rightarrow X$ can be deformed to a diffeomorphism which moves points only along the fibers. 
Proof. In 6.11 we observed that (2) above implies that Out $(a)=0$. Here $a$ is the extension $1 \rightarrow \mathbf{Z}^{k} \rightarrow \mathbf{Z}^{k}{ }_{\gamma} \mathbf{Z} \rightarrow \mathbf{Z} \rightarrow 1$. Thus $K=$ Out $\left(\pi ; \mathbf{Z}^{k}\right)$. To insure Out $\left(\pi ; \mathbf{Z}^{k}\right)=$ Out $(\pi)$ it suffices to have $\mathbf{Z}^{k}$ a characteristic subgroup of $\pi=\mathbf{Z}^{k}{ }_{\gamma} \mathbf{Z}$. This is implied by $f(1)=\operatorname{det}(I-\gamma) \neq 0$ which completes the proof.

We have shown that the homomorphism $G_{1}\left(T^{k}, X, \phi\right) \rightarrow$ Out $\pi$ is an epimorphism.

In [13, Theorem 2] we proved by a more sophisticated analysis that this epimorphism splits. By additional hypotheses such as no eigenvalues equal to roots of unity one can prove that $\pi$ has trivial center. Then, $i: G_{1}\left(T^{k}, X, \phi\right)$ $\rightarrow \mathcal{E}(X)$ is a homotopy equivalence.

\section{Deforming homotopy equivalences to fiber preserving homeomorphisms.}

8.1. In this section, we examine the realization problem for the full outer automorphism group, Out $\pi$. We have defined a significant subgroup $G\left(T^{k}, X, \phi\right)$ of the group of homeomorphisms $\mathcal{H}(X)$ of a Seifert fibering $\left(T^{k}, X, \phi\right)$. We shall characterize when this group of fiber preserving homeomorphisms, $G\left(T^{k}, X, \phi\right)$, is mapped, under $\Psi \circ i$, homomorphically onto Out $(\pi)$. We shall then list numerous examples $\left(T^{k}, X, \phi\right)$ for which $\Psi \circ i$ : $G\left(T^{k}, X, \phi\right) \rightarrow$ Out $(\pi)$ is a surjection.

Let us now consider $(W, N)$. For the particular $a \in H_{\phi}^{2}\left(N ; \mathbf{Z}^{k}\right)$ take all pairs $(\hat{h}, \Phi)$, where $\Phi \in$ Aut $N$ and $\hat{h}: W \rightarrow W$ is a homeomorphism so that

(i) $\hat{h}(w \alpha)=\hat{h}(w) \Phi(\alpha)$,

(ii) $\Phi \in$ Out $(a) \subset$ Out $(N)$.

This set of pairs form a group under $\left(\hat{h}_{1}, \Phi_{1}\right)\left(\hat{h}_{2}, \Phi_{2}\right)=\left(\hat{h}_{1} \circ \hat{h}_{2}, \Phi_{1} \circ \Phi_{2}\right)$. For any such pair $(\hat{h}, \Phi)$, since $\Phi \in$ Aut $(a)$, there exists $g \in \mathrm{GL}(k, \mathbf{Z})$ so that $g(\phi(\alpha))(t)=(\phi \circ \Phi(\alpha)) g(t)$ and $g_{*}(a)=\Phi^{*}(a)$. Hence, choose $\lambda: W \rightarrow T^{k}$ so that

$$
g(m(w, \alpha))=(\phi(\Phi(\alpha)))(\lambda(w \alpha)) m(\hat{h}(w), \Phi(\alpha))(\lambda(w))^{-1} .
$$

Define $(g, h) \in G\left(T^{k}, X^{\prime}, \phi\right)$ by $(t, w) \rightarrow(g(t) \lambda(w), \hat{h}(w))$. One checks that 1 and 2 hold and so define $\nu^{\prime}(g, h) \in G\left(T^{k}, X, \phi\right)$ by $\langle(t, w)\rangle \rightarrow\langle g(t) \lambda(w), \hat{h}(w)\rangle$. Thus, combining the above with our Lemma 7.4 we have the following.

Theorem 10. Let $\bar{\Phi} \in$ Out $(a) \subset$ Out $(N)$; then $\bar{\Phi}$ lies in the image of the composite $G\left(T^{k}, X, \phi\right) \rightarrow$ Out $(\pi) \rightarrow$ Out $(a)$, if and only if, for some representative automorphism $\Phi$ there is a homeomorphism $\hat{h}: W \rightarrow W$ with $\hat{h}(w \alpha)$ $=\hat{h}(w) \Phi(\alpha)$, for all $w \in W$ and $\alpha \in N$.

We observe that if the homeomorphism $\hat{h}$ exists for some one representative $\Phi$ of $\bar{\Phi} \in$ Out $(a)$ then, in fact, it exists for all representatives. For if $\Phi^{\prime}(\alpha)=\beta \Phi(\alpha) \beta^{-1}$ for some $\beta \in N$, then define $\hat{h}^{\prime}(w)=h(w) \beta^{-1}$. Now

$$
\begin{aligned}
\hat{h}^{\prime}(w \alpha) & =\hat{h}(w \alpha) \beta^{-1}=\hat{h}(w) \Phi(\alpha) \beta^{-1} \\
& =\left(\hat{h}(w) \beta^{-1}\right)\left(\beta \Phi(\alpha) \beta^{-1}\right)=\hat{h}^{\prime}(w) \Phi^{\prime}(\alpha) .
\end{aligned}
$$

Combining this with Theorem 9 we have

8.2. COROLLARY. The homomorphism of the group of fiber preserving homeo- 
morphisms $\Psi \circ i: G\left(T^{k}, X, \phi\right) \rightarrow$ Out $\left(\pi ; \mathbf{Z}^{k}\right)$ is surjective, if and only if, for each $\Phi \in$ Aut $(N)$ representing an element of Out $(a)$ there is a homeomorphism $\hat{h}: W \rightarrow W$ with $\hat{h}(w \alpha)=\hat{h}(w) \Phi(\alpha)$.

If $\mathbf{Z}^{k}$ is a characteristic subgroup of $\pi$ then Out $\left(\pi ; \mathbf{Z}^{k}\right)$ can be replaced by Out $(\pi)$; cf. (6.3 and 6.14).

8.3. VERIFYING THE HYPOTHESIS OF THE COROLLARY. We now wish to describe certain situations for which the hypothesis of the corollary is satisfied.

(a) A trivial, but significant, case occurs when $N$ is finite and the action $W \times N \rightarrow W$ is trivial. Then for any $\Phi \in$ Aut $N$, define $\hat{h}=\mathrm{id}$ on $W$. If $\phi$ is faithful, then $\mathbf{Z}^{k}$ will be characteristic and all of Out $(\pi)$ can be geometrically realized. In particular, if $W=$ a point, then $X=T^{k} / N$ is a closed flat manifold and every homotopy equivalence of $X$ into itself can be deformed to a diffeomorphism. Actually we see that given an automorphism of $\pi$ we obtain $g \in \mathrm{GL}(k, \mathbf{Z}), \Phi \in$ Aut $(a) \subset$ Aut $N$ and a $\lambda \in T^{k}$ so that the homeomorphism $t \rightarrow g(t) \lambda$ represents an element of $G\left(T^{k}, X^{\prime}, \phi\right)=G\left(T^{k}, T^{k}, \phi\right)$. This is clearly an affine diffeomorphism of the $k$-torus with respect to the usual Riemannian connection. This yields, by projection, $\langle t\rangle \rightarrow\langle g(t) \lambda\rangle$ an element of $G\left(T^{k}, T^{k} / N, \phi\right)=G\left(T^{k}, X, \phi\right)$. Such a representative diffeomorphism on $X=T^{k} / N$ is an affine diffeomorphism of $X$. Thus the Lie group of affine diffeomorphisms of $(X)$, Aff $(X)$, is mapped epimorphically onto Out $(\pi)$. We shall see in (9.9) that the kernel is precisely the connected component of the identity. This yields our version of Charlap's and Vasquez' Theorem 1 [9].

The reader may also wish to examine the conclusion of our Theorem 8 in case $\pi$ is a torsion free extension arising from taking $W=$ a point, $N$ finite and $\phi: N \rightarrow \mathrm{GL}(k, \mathbf{Z})$. The group $\pi$ is, of course, the fundamental group of a flat manifold $T^{k} / N$. With a little effort the reader should be able to see that the conclusion of Theorem 8 , in this special case, is the same as (in fact, a refinement of), Charlap's and Vasquez' Theorem 2 in [9].

(b) Topologically RIGID ACTIONS. Let us say that an action $(W, N)$ is topologically rigid if given $\Phi: N \rightarrow N$ an automorphism, then the action $(W, N ; \Phi)$ defined by

$$
(w * \alpha)=(w) \Phi(\alpha)
$$

is equivariantly homeomorphic to $(W, N)$. That is, there exists a homeomorphism $\hat{h}: W \rightarrow W$ so that $\hat{h}(w \alpha)=\hat{h}(w) \Phi(\alpha)(=\hat{h}(w) * \alpha)$.

If $W$ is a smooth manifold and $(W, N)$ is smooth, then we may call $(W, N)$ smoothly rigid if $\hat{h}$ may be chosen to be a diffeomorphism.

We shall now list some well-known examples of topologically and smoothly rigid actions and we shall describe the corresponding Seifert fiber spaces.

(b)-1. Let $E(n)$ be the Euclidean group. This is the group of Euclidean motions on $\mathbf{R}^{n}$ with the usual Euclidean metric. It can be represented by the semidirect product of the vector group of translations, $V^{n}$, by the orthogonal group, $O(n)$. Let $N$ be a uniform discrete subgroup of $E(n)$, that is, $E(n) / N$ is compact. Such a group is called a crystallographic group. The group $N$ acts as isometries properly discontinuously on the homogeneous space $W$ $=O(n) \backslash E(n)$, isometric to $\mathbf{R}^{n}$ (with the usual metric). This action is free, if and only if, $N$ is torsion free, of course. A theorem of Bieberbach says that $N$ 
and $N_{1}$, two crystallographic subgroups of $E(n)$, are isomorphic if and only if they are conjugate by an element of the affine group $A(n)=V^{n} \circ \mathrm{GL}(n, \mathbf{R})$ (see $[45,3.2 .2])$. Thus, in particular, $(W, N)$ is smoothly rigid.

Each crystallographic group, $N$, which gives rise to Bieberbach classes gives rise to many Seifert fiberings other than just flat manifolds. For example, suppose $N=(\mathbf{Z} \times \mathbf{Z}) \circ \mathbf{Z} / 2 \mathbf{Z}$, where $\mathbf{Z} / 2 \mathbf{Z}$ acts on $\mathbf{Z} \oplus \mathbf{Z}$ by $(m, n) \rightarrow(-m$, $-n)$. Then

$$
H^{2}(N ; \mathbf{Z}) \simeq \mathbf{Z} \oplus(\mathbf{Z} / 2 \mathbf{Z} \oplus \mathbf{Z} / 2 \mathbf{Z} \oplus \mathbf{Z} / 2 \mathbf{Z}),
$$

when $\phi$ is trivial. There are an infinite number of closed aspherical 3-manifolds with different fundamental groups representing the different Bieberbach classes. Here it can easily be shown that only one of the Bieberbach classes gives rise to a manifold which possesses a flat structure. Thus knowing $N$ is smoothly rigid tells us that $G\left(S^{1}, X, \phi\right) \rightarrow$ Out $\left(\pi_{1},(X)\right)$ is surjective for an infinite number of Seifert fiber spaces, $X$, constructible from $N$. (To see that Out $\pi_{1}(X)=$ Out $\left(\pi_{1}(X) ; \mathbf{Z}\right)$ we need only observe that $N$ is centerless.) Moreover, observe that the smooth rigidity of this particular $N$ also guarantees the surjectivity of $G\left(T^{k}, X, \phi\right) \rightarrow$ Out $\left(\pi_{1}(X) ; \mathbf{Z}^{k}\right)$ for the myriad of other examples when we let $\phi$ and $k$ be arbitrary.

(b)-2. Let $N$ and $N^{\prime}$ be uniform discrete subgroups of a semisimple analytic Lie group $G$ which has no compact factors nor any 3-dimensional factors and trivial center (more generally we can assume that the closed discrete group $N$ is such that $G / N$ has finite volume and there exists no (analytic) homomorphism of $G$ onto PSL $(2, \mathbf{R})$ with the image of $N$ discrete in PSL $(2, \mathbf{R}))$. Then, $K / G=W$ is a Riemannian symmetric space, where $K$ is a maximal compact subgroup. A theorem of $D$. Mostow (and extensions by G. Prasad) says that if $\Phi: N \rightarrow N^{\prime}$ is an isomorphism then there exists an automorphism $\bar{\Phi}: G \rightarrow G$ so that $\left.\bar{\Phi}\right|_{N}=\Phi$ (see [34, 24.1 and 24.2]). This strong rigidity theorem can be used to show that $(W, N)$ is smoothly rigid as follows. Let $\Phi: N \rightarrow N$ be an automorphism. The extension $\bar{\Phi}$ may not be an inner automorphism of $G$ (which would immediately yield smooth rigidity) so we observe that Aut $(G)$ is a finite extension of $G=\operatorname{Inn}(G)$ by Out $(G)$, from the classification theory.

Let Aut $(G)=G_{1}$ be the corresponding Lie group. Let $K_{1}$ be a maximal compact subgroup of $G_{1}$ projecting onto $K$ under the homomorphism $G_{1} \rightarrow G$. The space $K_{1} \backslash G_{1}$ is naturally isomorphic to $K \backslash G=W$ and $G_{1}$ acts on $K_{1} \backslash G_{1}=K \backslash G$. Now the right actions $(K \backslash G, N),(K \backslash G, \Phi(N))$ are equivalent by a diffeomorphism lying in $G_{1}$. That is, $\bar{\Phi}: G \rightarrow G$ is an element of $G_{1}$, and the diffeomorphism is given by $\bar{h}(g)=\bar{\Phi} g \bar{\Phi}^{-1}$ in $G_{1}$. Since $G$ is normal in $G_{1}, \bar{\Phi} g \bar{\Phi}^{-1} \in G$. If we define $\hat{h}\left(K_{1} g_{1}\right)=K_{1} g_{1} \bar{\Phi}^{-1}$, then

$$
\begin{aligned}
\hat{h}\left(K_{1} g_{1} \gamma\right) & =K_{1} g_{1} \gamma \bar{\Phi}^{-1}=\left(K_{1} g_{1} \bar{\Phi}^{-1}\right)\left(\bar{\Phi} \gamma \bar{\Phi}^{-1}\right) \\
& =\hat{h}\left(K_{1} g_{1}\right) \bar{\Phi}(\gamma)=\hat{h}\left(K_{1} g_{1}\right) \Phi(\gamma) .
\end{aligned}
$$

Thus $(W, N)=(K \backslash G, N)=\left(K_{1} \backslash G_{1}, \Phi(N)\right)$ are conjugate in the slightly larger group $G_{1}$ of diffeomorphisms of the homogeneous space $K \backslash G$.

(b)-3. Let $G=W$ be a simply connected solvable Lie group, and $N$ a uniform discrete subgroup (more generally, let $N$ be a lattice in $G$ ). $G$ is diffeomorphic to $\mathbf{R}^{n}$. A famous theorem of Mostow states that $(W, N)$ is smoothly rigid. Note 
that in this case $N$ is necessarily torsion free. A special case concerns $N$ being a torsion free, finite generated, nilpotent group. Then a theorem of $\mathrm{Mal}^{\prime} \mathrm{cev}$ states that $N$ is a uniform discrete subgroup of some unique (up to isomorphism) analytic, simply connected, nilpotent Lie group. Furthermore, any two isomorphic copies of $N$ in $G$ are conjugate.

(b)-4. Let $W=\mathbf{R}^{2}$ and $N$ a properly discontinuous effective group of orientation preserving homeomorphisms of $\mathbf{R}^{2}$. Then $N$ is topologically equivalent to a properly discontinuous action of a discrete subgroup of the Euclidean group $E(2)$ on the Euclidean plane or a discrete subgroup of the linear fractional transformations PSL $(2, \mathbf{R})$ of the hyperbolic plane. Thus, up to topological equivalence, any action $(W, N)$ with $W / N$ compact can be regarded as a uniform action (SO (2) $\backslash E(2), N)$ or (SO (2) $\backslash$ PSL $(2, \mathbf{R}), N$ ) with $N$ crystallographic or Fuchsian. It is known that $N$ is differentiably rigid when $N$ is Fuchsian [31] and we have already observed this for $N$ crystallographic. Therefore $\left(\mathbf{R}^{2}, N\right)$ is topologically rigid.

A similar result also holds if some elements of $N$ reverse orientation.

8.4. The corollary of 8.2 and 8.3 yields a great deal of information concerning Problem 1-C. In the examples cited, all Bieberbach classes give rise to aspherical manifolds. In fact, $\pi_{0}(\mathcal{E}(X))=$ Out $\left(\pi_{1}(X)\right)$ and in cases (a), (b)-2, and (b)-4 it is easy to see that Out $\left(\pi ; \mathbf{Z}^{k}\right)=$ Out $\pi$ by using 6.3. We have, in particular,

THEOREM 11. Let $X$ be a closed aspherical manifold arising as a Bieberbach class from a rigid action ( $W, N$ ) of type (a), (b)-2 or (b)-4; then every selfhomotopy equivalence may be deformed to a fiber preserving diffeomorphism.

For $X$ of type (b)-1 and (b)-3 every self-homotopy equivalence which projects to Out $\left(\pi ; \mathbf{Z}^{k}\right)$ may also be deformed to a fiber preserving homeomorphism.

The manifolds of type (b)-4 have been exhaustingly treated in $[17, \S 12]$. When $k=1$, recall $(5.8(\mathrm{e}))$ that they are the well-known classical closed Seifert 3-manifolds [39] (except for the cases where a classical Seifert manifold has fundamental group finite, infinite cyclic or $\mathbf{Z} \circ \mathbf{Z} / 2 \mathbf{Z}$, which correspond to those whose universal covering is the 3 -sphere, or $S^{2} \times \mathbf{R}^{1}$. For all other cases the classical Seifert manifold is aspherical. If orientation reversing $\left(\mathbf{R}^{2}, N\right)$ are considered our fiberings are more general than the classical Seifert fiberings. F. Waldhausen and, independently, P. Orlik, E. Vogt and H. Zieschang had proved earlier that every homotopy equivalence of a classical Seifert 3manifold deforms to a fiber preserving diffeomorphism by quite different methods. By extending these methods, $H$. Zieschang has announced a similar result for the class (b)-4 [46]. When $k=2$ in (b)-4 the manifolds include all the elliptic surfaces $S$ of Kodaira [28] for which the defining holomorphic map $S$ onto the algebraic curve has no exceptional fibers and which have no covering of the type $S^{1} \times S^{3}$ or $T^{2} \times S^{2}$. That is, they correspond to elliptic surfaces which are aspherical. Such an elliptic surface must occur exactly when $N$ is orientation preserving and $\phi: N \rightarrow \mathrm{GL}(2, \mathbf{Z})$ lies in GL $(1, \mathbf{C})$. The elliptic surfaces diffeomorphic to algebraic elliptic surfaces corresponding exactly to Bieberbach classes of finite order. Furthermore, the arguments used to obtain the corollary can be refined to show that all the manifolds constructed in examples (a), (b)-2, and the Fuchsian case of (b)-4 are 
diffeomorphic if and only if, they have isomorphic fundamental groups. While we will not go into this point at this time, it does settle Problem 1-B within the class of manifolds constructed in the examples. Unfortunately, this does not say much about whether there exist other manifolds of the same homotopy type which are not Seifert manifolds. As mentioned earlier, the answer to this question in case (b)-4 with $k=1$ is negative, if and only if, the Poincare conjecture, in dimension 3 , is true.

9. The domination of $\mathcal{E}(M)$ by $\mathcal{F}(M)$ and the geometric realization of finite subgroups of Out $\pi$.

9.1. In $\$ 7$ we cited that for almost all $k$-torus bundles $M$ over the circle the epimorphism $G_{1}\left(T^{k}, M, \phi\right) \rightarrow$ Out $\left(\pi_{1}(M)\right)$ splits and that $i: G_{1}\left(T^{k}, M, \phi\right)$ $\rightarrow \mathcal{E}(M)$ is a homotopy equivalence. In general, for any space $X$, if we can find a subgroup $G_{1}$ of $\mathcal{H}(X)$ so that under the inclusion $i$, restricted to $G_{1}$, the (restricted) $i$ is a homotopy equivalence between $G_{1}$ and $\mathcal{E}(X)$, then $\mathcal{H}(X)$ $\stackrel{i}{\rightarrow} \mathcal{E}(X)$ is a domination since the composition $\mathscr{E}(X) 1 \leftrightarrows i\left(G_{1}\right) \rightarrow \mathcal{H}(X)$ $\stackrel{i}{\rightarrow} \mathcal{E}(X)$ is homotopic to the identity. In particular,

$$
\pi_{i}\left(\mathcal{F}(X), 1_{X}\right) \stackrel{i_{*}}{\longrightarrow} \pi_{i}\left(\mathcal{E}(X), 1_{X}\right)
$$

is an epimorphism, for all $i \geqslant 0$.

In this section we will establish the domination for the examples at the end of the last section. Our method is to re-examine $G\left(T^{k}, X, \phi\right)$ and embed in $G\left(T^{k}, X, \phi\right)$ a toral action $T^{s}$. We will show that the evaluation homorphism has image exactly the center of $\pi_{1}(X)$. However, the additional splitting of the epimorphism $\mathcal{H}(X) \rightarrow$ Out $\pi_{1}(X)$, is only partially settled for these cases. In fact an affirmative solution to the splitting problem has many significant consequences. We have investigated in [13] a somewhat simpler problem:

Problem 10. If $F$ is a finite subgroup of Out $\pi_{1}(X)$, where $\left(T^{k}, X, \phi\right)$ is a Seifert fiber space, does there exist $F_{1}$, a finite subgroup of $\mathcal{H}(X)$, so that $\Psi \circ i: F_{1} \rightarrow F \subset$ Out $\left(\pi_{1}(X)\right)$ is an isomorphism?

This problem makes sense for any space and is especially meaningful when $\pi_{0}(\mathscr{E}(X))$ replaces Out $\left(\pi_{1}(X)\right)$. The significance of this problem, in terms of number theory, manifold theory and group theory, for an arbitrary aspherical manifold is discussed in numerous places. See, for example, Serre [40], Johnson and Wall [27], Conner and Raymond [17], and K. Brown [7].

For 2-manifolds, the answer is known to be affirmative if $F$ is solvable (see [47]), but unknown for arbitrary $F$. Recently, Raymond and Scott [37] have shown that there exist aspherical Seifert manifolds $\left(T^{k}, M^{n}, \phi\right)$ and cyclic $F$ so that $F$ cannot be lifted back into $\mathcal{H}(X)$, for all $n \geqslant 3$. The aspherical manifolds in [37] are actually nil-manifolds. Self-diffeomorphisms $H$ are constructed whose nth power are diffeotopic to the identity but there are no homeomorphisms $K$ homotopic to $H$ so that $K^{n}$ is the identity.

We shall, nevertheless, answer Problem 10 affirmatively for all finite subgroups $F \subseteq \bar{H}_{\phi}^{1}\left(N ; \mathbf{Z}^{k}\right) \subseteq$ Out $(\pi)$. This will follow from the proof that in injective Seifert fiber spaces $\left(T^{k}, X, \phi\right)$, there exists an injective toral action $\left(T^{s}, X\right)$ where $\operatorname{ev}_{*}^{x_{0}}: \pi_{1}\left(T^{s}, 1\right) \rightarrow \pi_{1}\left(X, x_{0}\right)$ has image $Z(\pi) \cap \mathbf{Z}^{k}$.

9.2. $H_{\phi}^{0}\left(N ; T^{k}\right)$ AS A SUBGRoup OF $\mathcal{H}(X)$. Let us take an injective Seifert fiber space $\left(T^{k}, X, \phi\right)$. Since $\phi: N \rightarrow \operatorname{GL}(k, Z)$, the group $N$ acts on $T^{k}$ by 
$(\alpha, t) \rightarrow \phi(\alpha) t$. The fixed point set, $G=H_{\phi}^{0}\left(N ; T^{k}\right)$, is a closed subgroup of $T^{k}$. Let $G_{0}$ be the connected component of the identity. It is a subtorus of dimension $s$. We can find a splitting of $T^{k}$ into $T^{s} \times T^{k-s}$ so that $G$ $=G_{0} \times G / G_{0}$ with $G_{0}=T^{s}$ and $G / G_{0} \subset T^{k-s}$. Let $N_{1}$ be the stabilizer of all $W$ and put $N_{0}=N_{1} \cap($ kernel $\phi)$.

THEOREM 12. The group $G$ acts on $X$ so that $\mathrm{ev}_{*}^{x_{0}}: \pi_{1}\left(T^{s}, 1\right) \rightarrow \pi_{1}\left(X, x_{0}\right)$ has image $\mathscr{Z}(\pi) \cap \mathbf{Z}^{k}$. The finite group $G / G_{0}$ maps isomorphically onto the torsion subgroup of $H_{\phi}^{1}\left(N ; \mathbf{Z}^{k}\right)$.

Moreover, $G_{0}$ acts injectively and acts effectively as the s-torus $G_{0} / N_{0} \cap G_{0}$; $G / G_{0}$ acts effectively as $\left(G / G_{0}\right) /\left(G / G_{0}\right) \cap N_{0}$ and, as such, represents the image of $G / G_{0}$ in $\bar{H}_{\phi}^{1}\left(N ; \mathbf{Z}^{k}\right)$.

Proof. We may choose $T^{s} \times T^{k-s}$ so that $i_{*}: \pi_{1}\left(T^{s}\right) \rightarrow \pi_{1}\left(T^{k}\right)$ corresponds to $H_{\phi}^{0}\left(N ; \mathbf{Z}^{k}\right)$. We can see this from the exact sequence

$$
0 \rightarrow H_{\phi}^{0}\left(N ; \mathbf{Z}^{k}\right) \rightarrow H_{\phi}^{0}\left(N ; \mathbf{R}^{k}\right) \rightarrow H_{\phi}^{0}\left(N ; T^{k}\right) \stackrel{\delta}{\rightarrow} H_{\phi}^{1}\left(N ; \mathbf{Z}^{k}\right) .
$$

Note that $G / G_{0}$ is mapped by $\delta$ isomorphically onto the torsion subgroup of $H_{\phi}^{1}\left(N ; \mathbf{Z}^{k}\right)$ and that $\delta$ maps $G_{0}$ trivially. Each element of $H_{\phi}^{0}\left(N ; T^{k}\right)$ can be regarded as a continuous function $\lambda: W \rightarrow T^{k}$ so that $\lambda$ is constant on $W$ with value an element of $T^{k}$ that is left fixed by the action of $N$. That is,

$$
\phi(\alpha)(\lambda(w \alpha))=\lambda(w), \text { for all } \alpha \in N .
$$

Thus $\lambda \in G$ is an element of $H_{\phi}^{0}\left(N\right.$; MAPS $\left.\left(W, T^{k}\right)\right)$. Therefore, the action of $G \subseteq T^{s} \times T^{k-s}=T^{k}$ commutes with the action of $N$ on $X^{\prime}$ and we obtain an action of $G$ on $X^{\prime} / N=X$. It is clear that $\mathrm{ev}_{*}^{x_{0}}: \pi_{1}\left(T^{s}, 1\right) \rightarrow \pi_{1}\left(X, x_{0}\right)$ is precisely $\mathbf{Z}^{s} \subseteq \mathbf{Z}^{k}$.

We shall now show that $G$ acts effectively as $G / G \cap N_{0}$. Suppose $\lambda\langle t, w\rangle$ $=\langle\lambda t, w\rangle=\langle t, w\rangle$, for all $\langle t, w\rangle$. Then for a fixed $(t, w)$, there exists $\alpha \in N_{1}$ so that $(\lambda t, w) \alpha=(t, w)$. Thus, as $\lambda$ itself must be a covering transformation, it must be $\alpha^{-1}$. In particular, we have $\lambda \phi(\alpha)(t) m(w, \alpha)=t$, for all $t$. Hence for $t^{\prime} \in G$, we have $\phi(\alpha)\left(t^{\prime}\right)=t^{\prime}$. Consequently, $\lambda=m(w, \alpha)^{-1}$. Thus for arbitrary $t, \phi(\alpha)(t)=(t)$. Thus $\alpha \in$ kernel $\phi$ and hence an element of $N_{0}$. Conversely, an element of $G \cap N_{0}$ stabilizes all of $X$.

We now claim $\mathscr{Z}(\pi) \cap \mathbf{Z}^{k}=\mathbf{Z}^{s} \subseteq \mathbf{Z}^{k}$. For $(m, e) \in \mathscr{Z}(\pi)$ implies $m=$ $\phi(\alpha)(m)$, for all $\alpha \in N$, hence $\mathscr{Z}(\pi) \cap \mathbf{Z}^{k} \subset\left(\mathbf{Z}^{k}\right)^{N}=\mathbf{Z}^{s}$. Conversely, $(m, e)$ in $\mathbf{Z}^{s}$ implies $(m, e) \in \mathscr{Z}(\pi)$.

9.3. We may strengthen this theorem when $\mathscr{Z}(N) \cap($ kernel $\phi)$ is trivial to answer part of Problem 10.

Corollary 1. Let $\left(T^{k}, X, \phi\right)$ be an injective Seifert fiber space with $\mathscr{Z}(N)$ $\cap$ (kernèl $\phi)=1$. For any finite $F \subseteq H_{\phi}^{1}\left(N ; \mathbf{Z}^{k}\right) \subseteq$ Out $\pi$, there exists finite $F_{1} \subset \mathcal{H C}(X)$ so that $\Psi \circ i: F_{1} \rightarrow F$ is an isomorphism.

Furthermore, $\mathscr{Z}(\pi)=\mathbf{Z}^{s} \subseteq \mathbf{Z}^{k}$ and there exists an injective toral action $\left(T^{s}, X\right)$ so that $\mathrm{ev}_{*}^{x_{0}}\left(\pi_{1}\left(T^{s}, 1\right)\right)$ is the whole center $\mathbf{Z}^{s}$.

Proof. Since $\mathscr{Z}(N) \cap$ kernel $\phi=1, B_{\phi}^{1}=\bar{B}_{\phi}^{1}$. Hence,

$$
\bar{H}_{\phi}^{1}\left(N ; \mathbf{Z}^{k}\right)=H_{\phi}^{1}\left(N ; \mathbf{Z}^{k}\right) \text {. }
$$


Furthermore, we proved that $G / G_{0}$ is mapped isomorphically onto the torsion subgroup of $H_{\phi}^{1}\left(N ; \mathbf{Z}^{k}\right)$. We claim that $G / G_{0}$ acts effectively on $X$. The reason is that $N_{0} \cap G$ must also be a central element of $N$ as the reader may wish to check. Since, $\mathscr{Z}(N) \cap($ kernel $\phi)=1, N_{0} \cap G=1$ and $G_{0}$ and $G / G_{0}$ acts effectively. Moreover $G / G_{0}$, which is abelian, is the full torsion subgroup of $H_{\phi}^{1}\left(N ; \mathbf{Z}^{k}\right)$. Of course, $\mathscr{Z}(\pi)=\mathbf{Z}^{s}=\mathscr{Z}(\pi) \cap \mathbf{Z}^{k}$ since $\mathscr{Z}(N) \cap($ kernel $\phi)$ is assumed trivial.

Notice that the action in the theorem and the corollary is just along fibers.

To improve this result is a very delicate matter.

9.4. Domination of $\mathcal{E}_{0}(X)$ AND $\mathscr{E}(X)$. The second major application of Theorem 12 is the domination of $\varepsilon_{0}(X)$, the connected component of the identity of $\mathscr{E}(X)$, by the connected component of the identity, $\mathcal{K}_{0}(X)$, of $\mathcal{H}(X)$. Let $X$ be a compact $K(\pi, 1)$ and a Seifert fiber space $\left(T^{k}, X, \phi\right)$ so that $\mathcal{Z}(N) \cap($ kernel $\phi)$ is trivial. Then

Corollary 2. $i: \mathcal{F}_{0}(X) \rightarrow \varepsilon_{0}(X)$ is a domination. Furthermore, if $\Psi \circ i$ : $\mathcal{H}(X) \rightarrow$ Out $(\pi)$ is surjective, for example as in (a), (b)-2 or (b)-4 of 8.3, then $i: \mathcal{H}(X) \rightarrow \mathcal{E}(X)$ is a domination.

Proof. Our group $G_{0} \subset \mathcal{K}_{0}(X)$ is a torus $T^{s}$ and gives an action $\left(T^{s}, X\right)$ so that $\mathrm{ev}_{*}^{x_{0}}: \pi_{1}\left(T^{s}, 1\right) \rightarrow \pi_{1}\left(X, x_{0}\right)$ is an isomorphism onto the center of $\pi_{1}\left(X, x_{0}\right)$. By 3.1, this means that the embedding $T^{s} \hookrightarrow \mathscr{E}_{0}(X)$ is a homotopy equivalence. By the principle annunciated at the beginning of 9.1 this makes the inclusions $G_{0}\left(T^{k}, X, \phi\right)$ and $\mathcal{H}_{0}(X)$ dominations. Moreover, if $\Psi \circ i$ is surjective, then the inclusions $G\left(T^{k}, X, \phi\right) \hookrightarrow \mathcal{E}(X)$ and $\mathscr{H}(X) \hookrightarrow \mathcal{E}(X)$ are dominations.

9.5. HitTing the CenTer. We may as well point out another fact that partially solves Problem 3 . Let $\left(T^{k}, X, \phi\right)$ be an injective Seifert fibering so that $\mathscr{Z}(N) \cap$ kernel $\phi$ is trivial. We have shown that the center $\mathscr{Z}(\pi)$ is a free abelian group of rank $s$, and necessarily, an injective action $\left(T^{s}, X\right)$ exists for which $\mathrm{ev}_{*}^{x_{0}}\left(\pi_{1}\left(T^{s}, X\right)\right)$ is precisely the center of $\pi$. This means that the largest possible injective action that can occur actually must occur.

We have noticed that the existence of a Bieberbach class $a \in H_{\phi}^{2}\left(N ; \mathbf{Z}^{k}\right)$ and a corresponding Seifert fibering $\left(T^{k}, X, \phi\right)$ usually entails the existence of many more such classes $a^{\prime} \in H_{\phi}^{2}\left(N ; \mathbf{Z}^{k}\right)$ by $[14,3.3,3.4,3.5]$. Thus in case we have one aspherical $X$ with $\mathscr{Z}(N) \cap$ kernel $\phi=1$, then every aspherical Seifert fibering $\left(T^{k}, M, \phi\right)$ arising from any Bieberbach class in $H_{\phi}^{2}\left(N ; \mathbf{Z}^{k}\right)$ (note no dependence upon an explicit $(W, N)$ ) must have center of rank exactly $s$ and a "maximal" $\left(T^{s}, M\right)$ action. Therefore the homotopy type of $\mathcal{E}_{0}(M)$ does not depend upon the explicit Bieberbach class but only upon $\phi: N \rightarrow \mathrm{GL}(k, \mathbf{Z})$.

9.6. AN APPLICATION TO GROUP THEORY. We would like to illustrate how some of the geometric ideas developed herein can be used to solve a problem in group theory. Let $F \subset H_{\phi}^{1}\left(N ; \mathbf{Z}^{k}\right) \subset$ Out $(\pi)$ where $\pi$ satisfies $1 \rightarrow \mathbf{Z}^{k} \rightarrow \pi$ $\rightarrow N \rightarrow 1$ with $\mathscr{Z}(N) \cap$ kernel $\phi=1$ as above. We may ask if there exists an extension of the form $1 \rightarrow \pi \rightarrow E \rightarrow F \rightarrow 1$ with operators coming from $F \subset$ Out $\pi$.

COROllary 3. All such extensions exist.

Proof. Choose any Seifert fiber space $\left(T^{k}, X, \phi\right)$ with Bieberbach class $a \in H_{\phi}^{2}\left(N ; \mathbf{Z}^{k}\right)$ representing the given extension $1 \rightarrow \mathbf{Z}^{k} \rightarrow \pi \rightarrow N \rightarrow 1$. 
Such always exist by choosing for $W$ the universal covering of a space whose fundamental group is $N$. Corollary 1 constructs on $X$ an action of $F_{1}$ so that $\Psi \circ i: F_{1} \rightarrow F$ is an isomorphism onto. Thus an extension $1 \rightarrow \pi \rightarrow E \rightarrow F$ $\rightarrow 1$ does exist with prescribed operators. See $[17, \S 2]$ for an explicit construction of the group extension from the group action. Now all extensions can be constructed from this extension by the usual methods, e.g. [32, p. 128].

9.7. Our next application solves a very special but interesting case of a wellknown problem about Poincaré duality groups and aspherical manifolds. It also may be used to solve the corresponding special case of Serre's problem concerning torsion free groups of type VFL (virtual finite length) [40, p. 101]. See also [7].

Let $\left(T^{k}, M, \phi\right)$ be an aspherical manifold and an injective Seifert fibering with Bieberbach class $a \in H_{\phi}^{2}\left(N ; \mathbf{Z}^{k}\right)$ corresponding to the extension $1 \rightarrow \mathbf{Z}^{k}$ $\rightarrow \pi \rightarrow N \rightarrow 1$. For any finite group $F \subset H_{\phi}^{1}\left(N ; \mathbf{Z}^{k}\right) \subset$ Out $(\pi)$ with $\mathscr{Z}(N)$ $\cap$ kernel $\phi=1$, we constructed an effective group action $(F, M)$. This gives rise to an explicit group extension $1 \rightarrow \pi \rightarrow E \rightarrow F \rightarrow 1$. We may ask when is this extension free?

The extension is torsion free if and only if $(F, M)$ is free.

This is shown in $[17,2.4]$. Moreover, any action $\left(F_{1}, M\right)$ giving rise to an equivalent extension must also be free. Therefore, we have

COROLlARY 4. There exists an aspherical manifold whose fundamental group is $E$ if and only if the group $E$ in the extension $1 \rightarrow \pi \rightarrow E \rightarrow F \rightarrow 1$ is torsion free.

9.8. Since $(F, M)$ only moves along fibers it is relatively easy to determine whether the extension $E$ is free or not. Let $\lambda \in F \subseteq H_{\phi}^{1}\left(N ; \mathbf{Z}^{k}\right)$ and suppose $\lambda\langle t, w\rangle=\langle\lambda t, w\rangle=\langle t, w\rangle$. Then there exists $\alpha^{-1} \in N$ so that

$$
(\lambda t, w)=(t, w) \alpha^{-1} \text {. }
$$

That is, $t \chi(\alpha)=\lambda t$, and $\alpha^{-1} \in N_{w}$. This means that $\lambda$ is a value of the crossed homomorphism $\chi: N_{w} \rightarrow T^{k}$ which determines the action on the fiber $\left(T^{k} \times w\right)$. Thus, if the order of $F$ were relatively prime to the orders of each of the $N_{w}$, then the action $(F, M)$ would be free. An important special case occurs when $(W, N)$ is free. Then, the action $(F, M)$ is always free.

(The following seems amusing. Recall that $G_{2}$ as defined in $\$ 7$ contains $F$ if $F \subset H_{\phi}^{1}\left(N ; Z^{k}\right)$. This part of $G_{2}$ actually commutes with the action of $N$ on $M^{\prime}$, and thus $M / F$, in this case, can be described by an element of $H_{\phi \times \varepsilon}^{2}\left(N \times F ; \mathbf{Z}^{k}\right)$, where $\varepsilon: F \rightarrow \operatorname{GL}(k, \mathbf{Z})$ is trivial. This "fibers" over $W / N$ for the action of $F$ on $W$ can be taken to be trivial. Thus $M / F$ is also an injective Seifert fibering where $E$ is torsion free. $M / F$ is probably homeomorphic to $M$.)

A class of specific examples has already been described in 6.11. These $k$ torus bundles over the circle have $H_{\phi}^{1}\left(\mathbf{Z} ; \mathbf{Z}^{k}\right)=\left(\mathbf{Z}^{k} /(I-\phi(1)) \mathbf{Z}^{k}\right)$, a finite subgroup of order equal to $|f(1)|$. Of course, this subgroup acts freely on $M$. In [13] we show that $M / F$ where $F \subset H_{\phi}^{1}\left(N ; \mathbf{Z}^{k}\right)$ is always diffeomorphic to $M$.

9.9. EXAMPLE. Closed flat manifolds. We shall obtain topologically some of the results of [9] (see $\$ \S 4.6 .3$ and 8.3). Let $N$ be a finite group and $\phi: N \rightarrow \mathrm{GL}(k, \mathbf{Z})$ a representation. If $a \in H_{\phi}^{2}\left(N ; \mathbf{Z}^{k}\right)$ is a Bieberbach class then the extension $1 \rightarrow \mathbf{Z}^{k} \rightarrow \pi \rightarrow N \rightarrow 1$ is torsion free. We can, without any 
loss of generality, assume that $\phi$ is a faithful representation. We form the associated Seifert fiber space by taking for $W$ a point, and the action of $N$ on $W$ to be trivial. We may choose a 1-cocycle $\tilde{m}$ representative in

$$
H_{\phi}^{1}\left(N ; \operatorname{MAPS}\left(\text { point, } T^{k}\right)\right)=H_{\phi}^{1}\left(N ; T^{k}\right)
$$

so that $\delta(\tilde{m})=a$, and define the free action $\left(T^{k}, N\right)$ with $\left(T^{k}, X, \phi\right)$ $=\left(T^{k}, T^{k} / N, \phi\right)$. The action of $N$ is a covering group of isometries and coincides, when the usual flat metric on $T^{k}$ is taken, with the holonomy group of the flat manifold $X$. In fact, clearly the Bieberbach classes of $H_{\phi}^{2}\left(N ; \mathbf{Z}^{k}\right)$ coincide with the closed flat manifolds with holonomy $N$.

Corollary 5. The finite subgroups $F$ of $H_{\phi}^{1}\left(N ; \mathbf{Z}^{k}\right)$ can be geometrically realized by finite groups of isometries. Moreover, $T^{s} \times G / G_{0}=G$ acts effectively as a group of isometries on $X$ so that $\Psi \circ i(G)=\bar{H}_{\phi}^{1}\left(N ; \mathbf{Z}^{k}\right)=H_{\phi}^{1}\left(N ; \mathbf{Z}^{k}\right)$ $=G / G_{0}$. Furthermore, the center $\mathscr{Z}(\pi)$ maps monomorphically into $H_{1}(X)$ with finite cokernel and the image of the evaluation homomorphism $\mathrm{ev}_{*}^{x_{0}}: \pi_{1}\left(T^{s}\right)$ $\rightarrow \pi_{1}\left(X, x_{0}\right)$ is precisely $\mathscr{Z}(\pi)$.

Proof. Since $\phi: N \rightarrow \mathrm{GL}(k, \mathbf{Z})$ is faithful Corollary 1 applies to realize $F \subset K$. Moreover, from the way we have made our realizations it is clear that they are isometries. Since $\mathbf{Z}^{k}$ is a maximal abelian subgroup of $\pi, \mathscr{Z}(\pi) \subset \mathbf{Z}^{k}$ and hence $\mathscr{Z}(\pi)=\mathbf{Z}^{s}=\left(\mathbf{Z}^{k}\right)^{N}=H_{\phi}^{0}\left(N ; \mathbf{Z}^{k}\right)$.

We check now that $H_{1}\left(T^{k} / N ; \mathbf{Z}\right)$ has rank $s$. We have that $\left(H_{1}\left(T^{k} ; Q\right)\right)^{N}$ $\rightarrow H_{1}\left(T^{k} / N ; Q\right)$ is an isomorphism. Consequently,

$$
\mathrm{ev}_{\#}^{1}: \pi_{1}\left(T^{s}, 1\right) \rightarrow \pi_{1}\left(T^{k}, 1\right) \rightarrow \pi_{1}\left(T^{k} / N\right) \rightarrow H_{1}\left(T^{k} / N ; \mathbf{Z}\right)
$$

is a monomorphism with finite cokernel. Thus the action $\left(T^{s}, X\right)$ is homologically injective and $s=\operatorname{rank} \mathscr{Z}(\pi)=\operatorname{dim} G=\operatorname{rank} H_{1}(X ; \mathbf{Z})$.

From the exact sequence

$$
0 \rightarrow\left(\mathbf{Z}^{k}\right)^{N} \rightarrow\left(\mathbf{R}^{k}\right)^{n} \rightarrow\left(T^{k}\right)^{N} \rightarrow H_{\phi}^{1}\left(N ; \mathbf{Z}^{k}\right) \rightarrow 0
$$

we see that $G / G_{0}=H_{\phi}^{1}\left(N ; \mathbf{Z}^{k}\right)$.

The fact $\left(T^{s}, X\right)$ is homologically injective will enable one to show that $\left(T^{s}, X\right)$ fibers equivariantly over $\left(T^{s}, T^{s} / \Delta\right)$ with fiber a flat closed manifold and finite abelian structure group $\Delta \subset T^{s}[12, \S 7],[45,3.63]$.

\section{REFERENCES}

1. Robert Bieri, Gruppen mit Poincaré-Dualitat, Comment. Math. Helv. 47 (1972), 373-396.

2. Robert Bieri and Beno Eckman, Groups with homological duality generalizing Poincaré duality, Invent. Math. 20 (1973), 103-124. MR 49 \#5204.

3. - Finiteness properties of duality groups, Comment. Math. Helv. 49 (1974), 74-83. MR 49 \#5205.

4. R. L. Bishop and B. O'Neill, Manifolds of negative curvature, Trans. Amer. Math. Soc. 145 (1969), 1-49. MR 40 \# 4891.

5. Edward M. Bloomberg, Manifolds with no periodic homeomorphisms, Trans. Amer. Math. Soc. 202 (1975), 67-78.

6. A. Borel, Seminar on transformation groups, Ann. of Math. Studies, no. 46, Princeton Univ. Press, Princeton, N.J., 1960. MR 22 \#7129. 
7. Kenneth S. Brown, Euler characteristics of discrete groups and G-spaces, Invent. Math. 27 (1974), 229-264.

8. J. B. Carrell, Holomorphically injective complex toral actions, Proc. Second Conference on Compact Transformation Groups, Part II, Lecture Notes in Math., vol. 299, Springer-Verlag, New York, 1972, pp. 205-236.

9. L. S. Charlap and A. T. Vasquez, Compact flat Riemannian manifolds. III: The group of affinities, Amer. J. Math. 95 (1973), 471-494.

10. P. E. Conner, Differentiable periodic maps, rev. ed., Ergebnisse der Mathematik und ihrer Grenzgebiete, Springer-Verlag, Berlin and New York (in preparation).

11. P. E. Conner and Frank Raymond, Actions of compact Lie groups on aspherical manifolds, Topology of Manifolds (Proc. Inst., Univ. of Georgia, Athens, Ga., 1969), Markham, Chicago, Ill., 1970, pp. 227-264. MR 42 \#6839.

12. - Injective actions of toral groups, Topology 10 (1971), 283-296. MR 43 \#6937.

13. - Realizing finite groups of homeomorphisms from homotopy classes of self-homotopyequivalences, Manifolds-Tokyo 1973, edited by Akio Hattori, Univ. of Tokyo Press, Tokyo, 1975, pp. 231-238.

14. - Holomorphic Seifert fiberings, Proc. Second Conference on Compact Transformation Groups, Part II, Lecture Notes in Math., vol. 299, Springer-Verlag, New York, 1972, pp. 124-204.

15. - Derived actions, Proc. Second Conference on Compact Transformation Groups, Part II, Lecture Notes in Math., vol. 299, Springer-Verlag, New York, 1972, pp. 237-310.

16. - Injective actions of the toral groups. II, Proc. Second Conference on Compact Transformation Groups, Part II, Lecture Notes in Math., vol. 299, Springer-Verlag, New York, 1972, pp. 109-123.

17. - Manifolds with few periodic homeomorphisms, Proc. Second Conference on Compact Transformation Groups, Part II, Lecture Notes in Math., vol. 299, Springer-Verlag, New York, 1972, pp. 1-75.

18. P. E. Conner, Frank Raymond and Peter Weinberger, Manifolds with no periodic maps, Proc. Second Conference on Compact Transformation Groups, Part II, Lecture Notes in Math., vol. 299, Springer-Verlag, New York, 1972, pp. 81-108.

19. Samuel Eilenberg and Tudor Ganea, On the Lusternik-Schnirelmann category of abstract groups, Ann. of Math. (2) 65 (1957), 517-518. MR 19, 52.

20. D. H. Gottlieb, A certain subgroup of the fundamental group, Amer. J. Math. 87 (1965), 840-856. MR 32 \#6454.

21. Olof Hanner, Some theorems on absolute neighborhood retracts, Ark. Mat. 1 (1951), 389-408. MR 13, 266.

22. H. Holmann, Seifertsche Faserrdume, Math. Ann. 157 (1964), 138-166. MR 30 \#587.

23. S. T. Hu, Homotopy theory, Pure and Appl. Math., vol. 8, Academic Press, New York, 1959. MR 21 \#5186.

24. F. E. A. Johnson, Manifolds of homotopy type $K(\pi, 1)$. I, Proc. Cambridge Philos. Soc. 70 (1971), 387-393. MR 44 \# 7542.

25. - On the first end invariants of an exact sequence, Matematika 22 (1975), 60-70.

26. - On a conjecture of C. T. C. Wall (to appear).

27. F. E. A. Johnson and C. T. C. Wall, On groups satisfying Poincaré duality, Ann. of Math. (2) 96 (1972), 592-598. MR 47 \#358.

28. K. Kodaira, Compact complex analytic surfaces. I, Ann. of Math. (2) 71 (1960), 111-152. MR 24 \# A2396.

29. H. Blaine Lawson, Jr. and Shing Tung Yau, Compact manifolds of nonpositive curvature, J. Differential Geometry 7 (1972), 211-228. MR 48 \# 12402.

30. Ronnie Lee and Frank Raymond, Manifolds covered by Euclidean space, Topology 14 (1975), 49-58.

31. A. M. Macbeath, Geometrical realization of isomorphisms between plane groups, Bull. Amer. Math. Soc. 71 (1965), 629-630. MR 31 \# 1374.

32. Saunders Mac Lane, Homology, Die Grundlehren der math. Wissenschaften, Band 114, Academic Press, New York; Springer-Verlag, Berlin, 1963. MR 28 \# 122.

33. Y. Matsushima, Holomorphic vector fields and the first Chern class of a Hodge manifold, J. Differential Geometry 3 (1969), 477-480. MR 42 \#8431.

34. G. D. Mostow, Strong rigidity of locally symmetric spaces, Ann. of Math. Studies, no. 78, Princeton Univ. Press, Princeton, N. J., 1973. 
35. Paik Kee Kim and Jeffrey L. Tollefson, Splitting the PL involutions on non-prime 3manifolds (to appear).

36. M. S. Raghunathan, Discrete subgroups of Lie groups, Ergebinesse der Mathematik und ihrer Grenzgebiete, Band 68 Springer-Verlag, New York, 1972.

37. Frank Raymond and Leonard Scott, The failure of Nielsen's theorem in higher dimensions, Arch. Math. (to appear).

38. Frank Raymond and Jeffrey L. Tollefson, Closed 3 -manifolds with no periodic maps, Trans. Amer. Math. Soc. 221 (1976), 403-418.

39. H. Seifert, Topologie drei-dimensionaler gefaserter Raume, Acta. Math. 60 (1933), 147-238.

40. Jean-Pierre Serre, Cohomologie des groupes discrets, Prospects in Math., Ann. of Math. Studies, no. 70, Princeton Univ. Press, Princeton, N.J., 1971, pp. 77-170.

41. John Stallings, The piecewise-linear structure of Euclidean space, Proc. Cambridge Philos. Soc. 58 (1962), 481-488. MR 26 \#6945.

42. Centerless groups-an algebraic formulation of Gottlieb's theorem, Topology 4 (1965), 129-134. MR 34 \#2666.

43. Friedhelm Waldhausen, On irreducible 3-manifolds which are sufficiently large, Ann. of Math. (2) 87 (1968), 56-88. MR 36 \#146.

44. C. T. C. Wall, The topological space-form problems, Topology of Manifolds (Proc. Inst., Univ. of Georgia, Athens, Ga., 1969), Markham, Chicago, Ill., 1970, pp. 319-331. MR 43 \#5540.

45. Joseph A. Wolf, Spaces of constant curvature, 2nd ed., Department of Mathematics, University of California, Berkeley, Calif., 1972. MR 49 \#7957.

46. H. Zieschang, On toric fibering over surfaces, Mat. Zametki 5 (1969), 569-576 = Math. Notes 5 (1969), 341-345. MR 40 \#2085.

47. - On extensions of fundamental groups of surfaces and related groups, Bull. Amer. Math. Soc. 77 (1971), 1116-1119; Addendum, ibid. 80 (1974), 366-367. MR 44 \# 4098.

Department of Mathematics, Louisiana State University, Baton Rouge Louisiana 70803

Department of Mathematics, University of Michigan, Ann ARbor, Michigan 48104 